\title{
Geometry of Spectral Curves and All Order Dispersive Integrable System
}

\author{
Gaëtan BOROT $^{\dagger}$ and Bertrand EYNARD $\ddagger \S$ \\ $\dagger$ Section de Mathématiques, Université de Genève, \\ 2-4 rue du Lièvre, 1211 Genève 4, Switzerland \\ E-mail: gaetan.borot@unige.ch \\ $\ddagger$ Institut de Physique Théorique, CEA Saclay, Orme des Merisiers, \\ 91191 Gif-sur-Yvette, France \\ E-mail: bertrand.eynard@cea.fr \\ $\S$ Centre de Recherche Mathématiques de Montréal, Université de Montréal, \\ P.O. Box 6128, Montréal (Québec) H3C 3J\%, Canada
}

Received November 14, 2011, in final form December 11, 2012; Published online December 18, 2012 http://dx.doi.org/10.3842/SIGMA.2012.100

\begin{abstract}
We propose a definition for a Tau function and a spinor kernel (closely related to Baker-Akhiezer functions), where times parametrize slow (of order $1 / N$ ) deformations of an algebraic plane curve. This definition consists of a formal asymptotic series in powers of $1 / N$, where the coefficients involve theta functions whose phase is linear in $N$ and therefore features generically fast oscillations when $N$ is large. The large $N$ limit of this construction coincides with the algebro-geometric solutions of the multi-KP equation, but where the underlying algebraic curve evolves according to Whitham equations. We check that our conjectural Tau function satisfies Hirota equations to the first two orders, and we conjecture that they hold to all orders. The Hirota equations are equivalent to a self-replication property for the spinor kernel. We analyze its consequences, namely the possibility of reconstructing order by order in $1 / N$ an isomonodromic problem given by a Lax pair, and the relation between "correlators", the tau function and the spinor kernel. This construction is one more step towards a unified framework relating integrable hierarchies, topological recursion and enumerative geometry.
\end{abstract}

Key words: topological recursion; Tau function; Sato formula; Hirota equations; Whitham equations

2010 Mathematics Subject Classification: 14H70; 14H42; 30Fxx

\section{Introduction}

Integrable systems are nonlinear dynamical systems, and in many cases, some exact solutions can be produced in terms of algebraic geometry of Riemann surfaces. For instance, Liouville integrable systems can be brought into the form of a linear constant motion with constant velocity in a multidimensional torus which is the Jacobian of some algebraic curve. However, not all solutions are algebro geometric, and an important question is how to find some solutions as perturbations of algebro-geometric ones.

\subsection{Goal and motivations}

Our goal is to propose a definition of a formal series for a perturbation of an algebro-geometric solution of an integrable system, in a "small" parameter which we call $1 / N$. Our definition consists in an all order expansion in powers of $1 / N$, whose leading order is the usual algebro-geometric 
solution, and whose corrections to each orders contain fast oscillating terms of frequency $N$, constructed from the invariants of [38].

The motivation for our definition, is to mimick the large $N$ expansion of random $N \times N$ matrix integrals.

Indeed, it is well known $[1,50,69,78]$ that random matrix models are particular examples of Tau-functions of some integrable systems, and also, the formal large $N$ expansion of matrix models can be obtained by formally solving Schwinger-Dyson equations (called loop equations in the context of matrix models), which leads to the invariants of [38], and to an expansion of the Tau-function in terms of them in [35, 37].

Therefore, in this paper, we propose to use the expressions introduced in $[35,37]$ for matrix models, in a lager context, as candidate Tau-functions associated to an arbitrary algebraic curve. We conjecture that the expression we propose does satisfy (formally) Hirota equation to all orders in $1 / N$. We prove it to the two first non-trivial orders.

As a motivation, we recall, that it was proved in [6] that our proposal retrieves the asymptotic expansion for the $(p, 2)$ minimal model reduction of $\mathrm{KdV}$. In the most general case, the matching between our construction and the asymptotic expansion of matrix models has not yet received a proof. However, in some cases like the one-hermitian matrix model with real potentials and some extra assumptions, it has been established to all orders $[3,34]$ in the one-cut regime (no modulation factors, pure $1 / N$ expansion) in [3, 19,34], and has been addressed up to $o(1)$ (including the modulation by a theta function) in multi-cut regimes in the works $[7,16,24,26]$.

In all examples above appeared a triple $\mathcal{S}=(\mathcal{C}, X, Y)$ consisting of a Riemann surface $\mathcal{C}$ and two functions $X$ and $Y$ defined on it, or some variants, like a curve and 2 meromorphic differentials $\mathrm{d} X$ and $Y \mathrm{~d} X$. We call this data a spectral curve, and it plays a central role in this article.

There exists several, non tautologically equivalent approaches to integrable systems (see [4, 32] for reviews). In this article, we take the notion of Tau function [53, 54, 56] as a starting point. It is a function of all the times which satisfies Hirota bilinear relations [47]; Tau functions are in correspondence with solutions of the nonlinear integrable PDE's.

Our main goal is to propose that the formal series defined in Definition 5.4 as a functional on the space of spectral curves, is a Tau function. We hope that it will allow the prediction of the full asymptotic expansion (in the small dispersive parameter $1 / N$ ) of some solutions of integrable PDE's in any genus $\mathfrak{g}$ regime, although we postpone precise comparisons to future works (see [64] for perturbation theory of Tau functions).

We just mention that the leading order of our construction retrieves the well-known asymptotic solutions of $\mathrm{KdV}$ in the genus 1 regime. More precisely, for such a comparison, we need to consider our construction to 1 -form $Y \mathrm{~d} X=x \omega_{\infty, 1}+2 t \omega_{\infty, 3}$ defined on the elliptic curve $\Upsilon^{2}=\prod_{i=1}^{3}\left(X-a_{i}(x, t)\right)$, where $a_{i}(x, t)$ satisfy Whitham type equations [81]

$$
\partial_{t} a_{i}=\frac{\omega_{\infty, 3}\left(a_{i}\right)}{\omega_{\infty, 1}\left(a_{i}\right)} \partial_{x} a_{i}
$$

and where $x$ (resp. $t$ ) is the time associated with the 1 -form $\omega_{\infty, 1}$ (resp. $2 \omega_{\infty, 3}$ ). We have used here the parametrization of meromorphic 1-forms introduced in Section 2.2, and $\infty$ denotes the point at infinity around which $\left.\Upsilon^{2} \sim X^{3}\right)$. The phase $\zeta(x, t)$ coincides with $\zeta(\mathbf{t})$ of Section 3.1 and $C$ a constant depending on the initial data. $\tau(x, t)$ is the time-evolved Riemann period of the elliptic curve, and $E_{2}$ the second Eisenstein series. For solutions of the KdV equation

$$
u_{t}+u u_{x}+\frac{1}{N^{2}} u_{x x x}=0
$$


with generic initial data, it has been proved $[25,42,45,66,67,68,79,80]$ that $u_{N}(x, t)$ for some time after the gradient catastrophe, $u_{N}(x, t)$ is asymptotic to (in a distributional sense)

$$
u_{N}(x, t)=\frac{2 \pi^{2} E_{2}(\tau(x, t))}{3}+\frac{1}{3} \sum_{i=1}^{3} a_{i}(x, t)+\frac{2}{N^{2}} \partial_{x}^{2} \ln \theta(N \zeta(x, t)+C \mid \tau(x, t)),
$$

where $\theta$ is a genus 1 Theta function. The relation between the Tau function and the solution of equation $(1.1)$ is $u(x, t)=2(\ln \mathcal{T})_{x x}$, and our candidate Tau function defined in Definition 5.4 indeed match in this setting the leading behavior equation (1.2).

We expect our proposal to be of interest in the study of asymptotics of solutions of hierarchies known to govern those matrix models like continuous Toda or nonlinear Schrödinger, in any finite genus regime. We also stress that matrix models are only special cases of our construction, in other words $\mathcal{T}[\mathcal{S}]$ is not in general a matrix integral. We hope that our general construction would describe the all-order asymptotics of solutions of the full dispersive hierarchies associated to Hurwitz spaces, although we do not attempt to make the comparison and do not address the hamiltonian formalism in this article.

\subsection{Outline of the article}

After a summary of algebraic geometry in Section 2, we review in Section 3 the reconstruction of an isospectral Lax system from its semiclassical spectral curve (which is time-independent). The techniques for this reconstruction are closely related to those developed by Krichever [62, 63] to produce the algebro-geometric solutions of the Zakharov-Shabat hierarchies [77]. We put emphasis on the Baker-Akhiezer spinor kernel $\psi_{\mathrm{cl}}\left(z_{1}, z_{2}\right)$ [59,60], and the corresponding Tau function $\mathcal{T}_{\mathrm{cl}}(\mathbf{t})$ in Section 4. Apart from fixing notations, this review is relevant to the present work, as one can illustrate in the case of $\mathrm{KdV}$ where the spectral curve does not depend on the times $x$ and $t$ (i.e. $a_{i}$ and $\tau$ are assumed constant) provides an exact solution of $\mathrm{KdV}[51,52]$ which can be obtained by such a reconstruction. Whereas, if one let $a_{i}(x, t)$ evolve according to Whitham equations as in the second part of the paper, it also describes the leading order of a solution of $\mathrm{KdV}$ in the small dispersion limit and for some time after the gradient catastrophe for generic initial data.

Then, for any spectral curve $\mathcal{S}$ whose time evolution is described by Whitham equations $[65,81]$ (cf. Section 5.1.2), we shall define explicitly in Section 5 a functional $\mathcal{T}[\mathcal{S}]$ (Definition 5.4) as a formal asymptotic series in a small parameter $N$, as well as a spinor kernel $\psi\left(z_{1}, z_{2}\right)$ via a Sato-like formula (Definition 5.5), which plays the role of a Baker-Akhiezer spinor kernel. We also introduce in Section 6 the correlators $W_{n}\left(z_{1}, \ldots, z_{n}\right)$ (Definition 6.2), which encode the $n$-th order derivatives of $\mathcal{T}[\mathcal{S}]$ with respect to deformation parameters of $\mathcal{S}$. Here, $z_{1}, \ldots, z_{n}$ denotes points on $\mathcal{C}$.

The essential point in this article is the conjecture that $\mathcal{T}[\mathcal{S}]$ satisfies a certain form of Hirota equations to all orders in $1 / N$ (Conjecture 7.4), and we check it holds for the two first orders (Appendix A). We present an equivalent conjecture stating that $\psi\left(z_{1}, z_{2}\right)$ is selfreplicating (Conjecture 7.1). This conjecture automatically implies determinantal formulas for the correlators (Theorem 8.1), Christoffel-Darboux formula for the spinor kernel (Theorem 8.3), and a Lax system satisfied by the matrix $\Psi\left(x_{1}, x_{2}\right)=\left[\psi\left(z^{i}\left(x_{1}\right), z^{j}\left(x_{2}\right)\right)\right]_{i, j}$, where $z^{i}(x) \in \mathcal{C}$ are the points such that $X\left(z^{i}(x)\right)=x$ (Section 8.6).

The coefficient of the so-obtained Lax matrices can be computed in principle order by order in $1 / N$. If our conjecture is correct, our approach describes directly a Tau function, but we do not identify the underlying nonlinear hierarchy of equations. The situation is similar to the one evoked in [33], where the dispersive hierarchy is constructed perturbatively in $1 / N$, but its resummation for finite $N$ is unknown - except in special cases. 


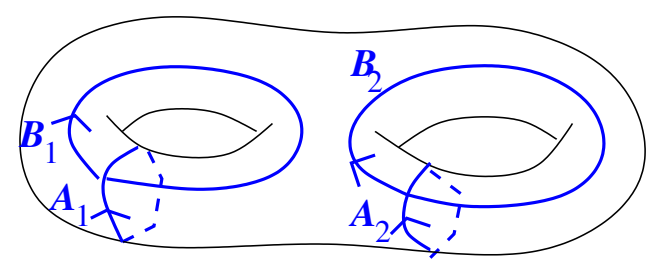

Figure 1. A symplectic basis of $2 \mathfrak{g}$ non-contractible cycles on a Riemann surface of genus $\mathfrak{g}$.

Since our approach was strongly motivated by earlier results or heuristics in hermitian matrix models, we recapitulate their relation to the present work in Section 9.

\section{Geometry of the spectral curve}

We briefly describe some geometric notions attached to a fixed spectral curve $\mathcal{S}=(\mathcal{C}, X, Y)$ $[31,40,41]$. To simplify, we assume in this article that $\mathcal{C}$ is a compact Riemann surface of genus $\mathfrak{g}$, and $X$ and $Y$ are meromorphic functions on $\mathcal{C}$.

\subsection{Some notations and properties}

\subsubsection{Topology and holomorphic 1-forms}

The curve $\mathcal{C}$ is either simply connected, and then this is the Riemann sphere $\mathcal{C}=\mathbb{C} \cup\{\infty\}$, or it has genus $\mathfrak{g}>0$. Then, any maximal open contractible subset of $\mathcal{C}$ is called a fundamental domain. If it is of genus $\mathfrak{g}>0$, there exist $2 \mathfrak{g}$ independent non-contractible cycles (see Fig. 1 ), and we can choose them in such a way (but not unique) that

$$
\mathcal{A}_{i} \cap \mathcal{B}_{j}=\delta_{i, j}, \quad \mathcal{A}_{i} \cap \mathcal{A}_{j}=0, \quad \mathcal{B}_{i} \cap \mathcal{B}_{j}=0 .
$$

A basis satisfying these intersection relations is called "symplectic".

From the topological point of view, a genus $\mathfrak{g}>0$ compact Riemann surface with a basis $\left(\mathcal{A}_{i}, \mathcal{B}_{i}\right)_{1 \leq i \leq \mathfrak{g}}$ is a $4 \mathfrak{g}$ closed polygon $\Gamma$, with edges

$$
\left[\mathcal{A}_{1}, \mathcal{B}_{1}, \mathcal{A}_{1}^{-1}, \mathcal{B}_{1}^{-1}, \ldots, \mathcal{A}_{\mathfrak{g}}, \mathcal{B}_{\mathfrak{g}}, \mathcal{A}_{\mathfrak{g}}^{-1}, \mathcal{B}_{\mathfrak{g}}^{-1}\right]
$$

glued by pairs. $\stackrel{\circ}{\Gamma}$ is a fundamental domain of $\mathcal{C}$. It is a classical result, that on a curve of genus $\mathfrak{g}$, there exists $\mathfrak{g}$ independent holomorphic 1-forms $\mathrm{d} u_{i}$ (holomorphic means in particular having no poles), and they can be normalized on the $\mathcal{A}$-cycles

$$
\oint_{\mathcal{A}_{i}} \mathrm{~d} u_{j}=\delta_{i, j}
$$

Then, the $\mathfrak{g} \times \mathfrak{g}$ matrix $\tau_{i, j}$,

$$
\tau_{i, j}=\oint_{\mathcal{B}_{i}} \mathrm{~d} u_{j}
$$

is known to be symmetric $\tau_{i, j}=\tau_{j, i}$ and its imaginary part is definite positive

$$
\tau^{t}=\tau, \quad \operatorname{Im} \tau>0
$$

$\tau$ is called the Riemann matrix of periods of $\mathcal{C}$. 


\subsubsection{Theta functions}

Given any symmetric matrix $\tau$ such that $\operatorname{Im} \tau>0$, one can define the Riemann Theta function

$$
\theta(\mathbf{u} \mid \tau)=\sum_{\mathbf{n} \in \mathbb{Z}^{\mathfrak{g}}} \mathrm{e}^{2 \mathrm{i} \pi \mathbf{n} \cdot \mathbf{u}} \mathrm{e}^{\mathrm{i} \pi \mathbf{n}^{t} \cdot \tau \cdot \mathbf{n}}
$$

Since $\operatorname{Im} \tau>0$, it is a well-defined convergent series for all $\mathbf{u}$ in $\mathbb{C}^{\mathfrak{g}}$. Most often we will not write the $\tau$ dependence of the Theta function: $\theta(\mathbf{u} \mid \tau) \equiv \theta(\mathbf{u})$. This function is quasi-periodic in $\mathbf{u}$ : if $\mathbf{n}, \mathbf{m} \in \mathbb{Z}^{\mathfrak{g}}$, we have

$$
\theta(\mathbf{u}+\mathbf{n}+\tau \mathbf{m})=\mathrm{e}^{-\mathrm{i} \pi\left(2 \mathbf{m}^{t} \cdot \mathbf{u}+\mathbf{m}^{t} \cdot \tau \cdot \mathbf{m}\right)} \theta(\mathbf{u}) .
$$

It also satisfies the heat equation

$$
\partial_{\tau_{i, j}} \theta=\frac{1}{4 \mathrm{i} \pi} \partial_{u_{i}} \partial_{u_{j}} \theta
$$

In this equation, $\tau_{i, j}$ and $\tau_{j, i}$ are considered independent.

\subsubsection{Jacobian and Abel map}

Let us choose a generic basepoint $o \in \mathcal{C}$ (it will in fact play no role). For any point $z \in \mathcal{C}$, we define

$$
\forall i \in\{1, \ldots, \mathfrak{g}\}, \quad u_{i}(z)=\int_{o}^{z} \mathrm{~d} u_{i},
$$

where the integration path is chosen such that it does not intersect any $\mathcal{A}$-cycle or $\mathcal{B}$-cycle. Then we define the vector

$$
\mathbf{u}(z)=\left[u_{i}(z)\right]_{1 \leq i \leq \mathfrak{g}} \in \mathbb{C}^{\mathfrak{g}} .
$$

The application $z \mapsto \mathbf{u}(z) \bmod \left(\mathbb{Z}^{\mathfrak{g}}+\tau \cdot \mathbb{Z}^{\mathfrak{g}}\right)$ is well-defined and analytical, it maps the spectral curve into the Jacobian $\mathbb{J}=\mathbb{C}^{\mathfrak{g}} /\left(\mathbb{Z}^{\mathfrak{g}}+\tau \cdot \mathbb{Z}^{\mathfrak{g}}\right)$. This defines the Abel map

$$
\begin{aligned}
\mathcal{C} & \rightarrow \mathbb{J}, \\
z & \mapsto \mathbf{u}(z) \bmod \left(\mathbb{Z}^{\mathfrak{g}} \oplus \tau \cdot \mathbb{Z}^{\mathfrak{g}}\right) .
\end{aligned}
$$

The Jacobi inversion theorem states that every $\mathbf{w} \in \mathbb{J}$ can be represented as $\mathbf{w}=\sum_{j=1}^{\mathfrak{g}} \mathbf{u}\left(p_{j}\right)$ for some points $p_{1}, \ldots, p_{\mathfrak{g}} \in \mathcal{C}$.

The Theta function can be used with $\tau$ the Riemann matrix of periods of a Riemann surface $\mathcal{C}$, and $\mathbf{u}$ the Abel map of a point on $\mathcal{C}$. In this case, it enjoys other important properties. Its zero locus has the following description: there exists $\mathbf{k} \in \mathbb{C}^{\mathfrak{g}}$, so that $\theta(\mathbf{w} \mid \tau)=0$ iff there exists $\mathfrak{g}-1$ points $z_{1}, \ldots, z_{\mathfrak{g}-1} \in \mathcal{C}$ satisfying $\mathbf{w}=\sum_{j=1}^{\mathfrak{g}-1} \mathbf{u}\left(z_{j}\right)+\mathbf{k}$. $\mathbf{k}$ is called a "Riemann vector of constants", and it depends on the basepoint $o$ used to define the Abel map u.

\subsubsection{Prime form}

An odd characteristics $\mathbf{c}$ is a vector of the form

$$
\mathbf{c}=\frac{\mathbf{n}+\tau \mathbf{m}}{2}, \quad \mathbf{n}, \mathbf{m} \in \mathbb{Z}^{\mathfrak{g}}, \quad \mathbf{n}^{t} \cdot \mathbf{m} \in 2 \mathbb{Z}+1 .
$$


The Theta function vanishes at odd characteristics: $\theta(\mathbf{c})=0$, and the following holomorphic form

$$
\mathrm{d} h_{\mathbf{c}}(z)=\sum_{i=1}^{\mathfrak{g}} \mathrm{d} u_{i}(z) \partial_{u_{i}} \theta(\mathbf{c})
$$

has only double zeroes on $\mathcal{C}$, so that we can define its squareroot, and thus one can define the prime form $[71,72,73]$

$$
E\left(z_{1}, z_{2}\right)=\frac{\theta\left(\mathbf{u}\left(z_{1}\right)-\mathbf{u}\left(z_{2}\right)+\mathbf{c}\right)}{\sqrt{\mathrm{d} h_{\mathbf{c}}\left(z_{1}\right) \mathrm{d} h_{\mathbf{c}}\left(z_{2}\right)}} .
$$

There exists choices of $\mathbf{c}$ such that $E$ is not identically 0 (we say $\mathbf{c}$ is "non singular"), and $E$ is in fact independent of such c. It is a $\left(-\frac{1}{2},-\frac{1}{2}\right)$-form on $\mathcal{C} \times \mathcal{C}$, and it vanishes only at $z_{1}=z_{2}$. In any local coordinate $\xi(z)$ we have

$$
E\left(z_{1}, z_{2}\right) \underset{z_{1} \rightarrow z_{2}}{=} \frac{\xi\left(z_{1}\right)-\xi\left(z_{2}\right)}{\sqrt{\mathrm{d} \xi\left(z_{1}\right) \mathrm{d} \xi\left(z_{2}\right)}}+O\left(\left(\xi\left(z_{1}\right)-\xi\left(z_{2}\right)\right)^{3}\right) .
$$

Because of the Theta function, $E\left(z_{1}, z_{2}\right)$ is multivalued $\mathcal{C} \times \mathcal{C}$. It transforms according to equation (2.1).

The Theta function associated to a Riemann surface satisfies a non-linear relation called Fay identity [41]: for any $z_{1}, z_{2}, z_{3}, z_{4} \in \mathcal{C}$, any $\mathbf{w} \in \mathbb{C}^{\mathfrak{g}}$,

$$
\begin{aligned}
\theta(\mathbf{w}+\mathbf{c}) \theta\left(\mathbf{u}_{12}+\mathbf{u}_{34}+\mathbf{w}+\mathbf{c}\right) \frac{E\left(z_{1}, z_{3}\right) E\left(z_{2}, z_{4}\right)}{E\left(z_{1}, z_{4}\right) E\left(z_{2}, z_{3}\right)} \frac{1}{E\left(z_{1}, z_{2}\right) E\left(z_{3}, z_{4}\right)} \\
=\frac{\theta\left(\mathbf{w}+\mathbf{u}_{12}+\mathbf{c}\right)}{E\left(z_{1}, z_{2}\right)} \frac{\theta\left(\mathbf{w}+\mathbf{u}_{34}+\mathbf{c}\right)}{E\left(z_{3}, z_{4}\right)}-\frac{\theta\left(\mathbf{w}+\mathbf{u}_{14}+\mathbf{c}\right)}{E\left(z_{1}, z_{4}\right)} \frac{\theta\left(\mathbf{w}+\mathbf{u}_{32}+\mathbf{c}\right)}{E\left(z_{3}, z_{2}\right)}
\end{aligned}
$$

where $\mathbf{u}_{j l}=\mathbf{u}\left(z_{j}\right)-\mathbf{u}\left(z_{l}\right)$.

\subsubsection{Bergman kernel}

We call Bergman kernel the "fundamental (1,1)-form of the second kind" [41], defined as

$$
B\left(z_{1}, z_{2}\right)=\mathrm{d}_{z_{1}} \mathrm{~d}_{z_{2}} \ln \left(\theta\left(\mathbf{u}\left(z_{1}\right)-\mathbf{u}\left(z_{2}\right)+\mathbf{c}\right)\right) .
$$

It is independent of the choice of a non-singular, odd characteristics $\mathbf{c}$. It is a globally defined, symmetric $(1,1)$-form, having a double pole at $z_{1}=z_{2}$ with no residue, and no other pole. It is normalized so that

$$
\oint_{\mathcal{A}_{i}} B(\cdot, z)=0, \quad \oint_{\mathcal{B}_{i}} B(\cdot, z)=2 \mathrm{i} \pi \mathrm{d} u_{i}(z) .
$$

Near $z_{1}=z_{2}$, it behaves, in any local coordinate $\xi(z)$, like

$$
B\left(z_{1}, z_{2}\right) \underset{z_{1} \rightarrow z_{2}}{=} \frac{\mathrm{d} \xi\left(z_{1}\right) \mathrm{d} \xi\left(z_{2}\right)}{\left(\xi\left(z_{1}\right)-\xi\left(z_{2}\right)\right)^{2}}+O(1) .
$$

We also define the fundamental 1-form of the third kind

$$
\mathrm{d} S_{z_{1}, z_{2}}(z)=\int_{z_{2}}^{z_{1}} B(\cdot, z)
$$


where the integration contour is chosen so that it does not intersect any $\mathcal{A}$-cycle or $\mathcal{B}$-cycle. It is a 1 -form in the variable $z$, and a function of the variable $z_{1}, z_{2}$, and it satisfies

$$
\oint_{\mathcal{A}_{j}} \mathrm{~d} S_{z_{1}, z_{2}}=0, \quad \oint_{\mathcal{B}_{j}} \mathrm{~d} S_{z_{1}, z_{2}}=2 \mathrm{i} \pi\left(u_{j}\left(z_{1}\right)-u_{j}\left(z_{2}\right)\right) .
$$

It has a simple pole at $z=z_{1}$ with residue +1 , a simple pole at $z=z_{2}$ with residue -1 , and no other pole. In other words, in any local coordinate $\xi(z)$

$$
\mathrm{d} S_{z_{1}, z_{2}}(z) \underset{z \rightarrow z_{1}}{\sim} \frac{\mathrm{d} \xi(z)}{\xi(z)-\xi\left(z_{1}\right)}, \quad \mathrm{d} S_{z_{1}, z_{2}}(z) \underset{z \rightarrow z_{2}}{\sim} \frac{-\mathrm{d} \xi(z)}{\xi(z)-\xi\left(z_{2}\right)} .
$$

Notice that in the variable $z$ it is globally defined for $z \in \mathcal{C}$ (it has no monodromy if $z$ goes around a non-contractible cycle), whereas in the variable $z_{1}$ (resp. $z_{2}$ ) it is defined only on the fundamental domain, it has monodromies when $z_{1}$ (resp. $z_{2}$ ) goes around a non-contractible cycle $\mathcal{B}_{j}$

$$
\mathrm{d} S_{z_{1}+\mathcal{B}_{j}, z_{2}}(z)=\mathrm{d} S_{z_{1}, z_{2}}(z)+2 \mathrm{i} \pi \mathrm{d} u_{j}(z), \quad \mathrm{d} S_{z_{1}, z_{2}+\mathcal{B}_{j}}(z)=\mathrm{d} S_{z_{1}, z_{2}}(z)-2 \mathrm{i} \pi \mathrm{d} u_{j}(z) .
$$

\subsubsection{Example in genus $\mathfrak{g}=1$}

When $\mathfrak{g}=1$, the Abel map is an isomorphism between $\mathcal{C}$ and $\mathbb{J}=\mathbb{C} / \mathbb{L}$ where we set $\mathbb{L}=\mathbb{Z}+\tau \mathbb{Z}$. The $\mathcal{A}$-cycle in $\mathbb{J}$ is the segment $[0,1[$, and the $\mathcal{B}$-cycle is the segment $[0, \tau[$. The Bergman kernel normalized on $\mathcal{A}$-cycles can be expressed as

$$
B\left(u_{1}, u_{2}\right)=\mathrm{d} u_{1} \mathrm{~d} u_{2}\left(\wp\left(u_{1}-u_{2} \mid \tau\right)+\frac{\pi^{2} E_{2}(\tau)}{3}\right)
$$

where $u_{1}, u_{2} \in \mathbb{J}, \wp$ is the Weierstrass function and $E_{2}$ the second Eisenstein series

$$
\begin{aligned}
& \wp(u \mid \tau)=\frac{1}{u^{2}}+\sum_{w \in \mathbb{L} \backslash\{0\}}\left(\frac{1}{(u+w)^{2}}-\frac{1}{w^{2}}\right), \\
& E_{2}(\tau)=\frac{3}{\pi^{2}}\left(\sum_{n \neq 0} \frac{1}{n^{2}}+\sum_{m \neq 0} \sum_{n \in \mathbb{Z}} \frac{1}{(n+m \tau)^{2}}\right) .
\end{aligned}
$$

\subsection{Parametrization of meromorphic 1-forms}

\subsubsection{Sheets, ramification and branchpoints, local coordinate patches}

If $\operatorname{deg} X=d$, then for every value $x$, there are $d$ points $z^{1}(x), \ldots, z^{d}(x)$ on the curve $\mathcal{C}$ such that $X\left(z^{i}(x)\right)=x . z^{i}(x)$ is sometimes called the preimage of $x$ in the $i$-th sheet.

Definition 2.1. We call "ramification points of order $k$ ", the zeroes of order $k \geq 1$ of the meromorphic 1-form $\mathrm{d} X$. If $a \in \mathcal{C}$ is a ramification point, the corresponding value $X(a)$ is called a branchpoint. All the other points $z \in \mathcal{C}$ at which $X(z)$ is analytical, are called "regular points".

Definition 2.2. We say that a branchpoint $x_{a}$ is simple if $X^{-1}\left(\left\{x_{a}\right\}\right)$ consists in $d-1$ points, one of them being a ramification point of order 1 , and all the remaining ones being regular points. 


\subsubsection{Definition of local coordinates}

Near a ramification point $a$ of order $k, \xi_{a}(z)=(X-X(a))^{1 /(k+1)}$ defines a local coordinate on the curve. Simple branchpoints play a special role in Sections 3.6, 5.1 and 6.1. For a simple branchpoint we have

$$
\xi_{a}(z)=\sqrt{X(z)-X(a)} .
$$

Since $X$ is a meromorphic function of degree $d$, it has $d$ poles with multiplicities, i.e. $\infty_{1}^{d_{\infty_{1}}}, \ldots$, $\infty_{s}^{d_{\infty_{s}}}$ with $\sum_{i} d_{\infty_{i}}=d$. Near $\infty_{i}$, a good local variable is

$$
\xi_{\infty_{i}}(z)=X(z)^{-1 / d_{\infty_{i}}}
$$

Besides, we will need to consider also poles of a meromorphic form $\omega$. If $p$ is a pole of $\omega$, but not a pole of $X$, neither a zero of $\mathrm{d} X$, a good local variable is

$$
\xi_{p}(z)=X(z)-X(p)
$$

In this case, the multiplicity of $p$ is $d_{p}=-1$. We shall now always use the local coordinates $\xi(z)$ defined above. Notice that they depend only on the function $X(z)$.

Definition 2.3. Given a meromorphic 1-form $\omega(z)$ which has no pole at ramification points, let us call

$$
\mathcal{P}=\{\text { poles of } \omega\}, \quad \mathcal{P}_{\infty}=\{\text { poles of } X\}, \quad \overline{\mathcal{P}}=\mathcal{P} \cup \mathcal{P}_{\infty}
$$

To any $p \in \overline{\mathcal{P}}$, we have associated a coordinate patch $\xi_{p}$ on $\mathcal{C}$ centered on $p$.

\subsubsection{Poles and times, filling fractions}

Following Krichever [65], we define

Definition 2.4. For any $p \in \overline{\mathcal{P}}$, we define the "times" near $p$ as the coefficient of the negative part of the Laurent series expansion of $\omega$ near $p$

$$
\omega(z) \underset{z \rightarrow p}{=} \sum_{j \geq 0} t_{p, j}\left(\xi_{p}(z)\right)^{-(j+1)} \mathrm{d} \xi_{p}(z)+O(1), \quad t_{p, j}=\operatorname{Res}_{z \rightarrow p} \omega(z) \xi_{p}(z)^{j} .
$$

We also write collectively $\vec{t}_{p}=\left[t_{p, j}\right]_{j \in \mathbb{N}}$ and $\vec{t}=\left(\vec{t}_{p}\right)_{p \in \overline{\mathcal{P}}}$. We also define the "filling fractions" (also called "conserved quantities"), associated to non-contractible cycles, by

$$
\epsilon_{i}=\frac{1}{2 \mathrm{i} \pi} \oint_{\mathcal{A}_{i}} \omega
$$

Notice that the times $t_{p, 0}=\operatorname{Res}_{p} \omega$ are not independent, because the sum of residues of $\omega$ must vanish,

$$
\sum_{p \in \overline{\mathcal{P}}} t_{p, 0}=0
$$

There is a form-cycle duality [65] 
Definition 2.5. To each time $t_{k}$, one can associate a differential meromorphic form $\omega_{k}(z)$, as well as a dual cycle $\omega_{k}^{*}$, and a dual orthogonal cycle $\omega_{k}^{* \perp}$

$$
t_{k} \longleftrightarrow \omega_{k}(z) \longleftrightarrow \omega_{k}^{*} \longleftrightarrow \omega_{k}^{* \perp}
$$

in such a way that

$$
\begin{aligned}
& \left.\frac{\partial \omega(z)}{\partial t_{k}}\right|_{X(z)}=\omega_{k}(z), \quad \omega(z)=\sum_{k} t_{k} \omega_{k}(z), \\
& \omega_{k}(z)=\int_{\omega_{k}^{*}} B(\cdot, z), \quad t_{k}=\int_{\omega_{k}^{* \perp}} \omega, \quad \omega_{i}^{*} \cap \omega_{j}^{* \perp}=\delta_{i, j} .
\end{aligned}
$$

The symbol $\left.\right|_{X(z)}$ means that we differentiate keeping the local coordinates $\xi_{p}(z)$ fixed (i.e. $X(z)$ fixed).

More explicitly we have

- Filling fractions $\epsilon_{i} \longrightarrow \omega_{j}=$ first kind differential

$$
\omega_{j}(z)=2 \mathrm{i} \pi \mathrm{d} u_{j}(z)=\oint_{\mathcal{B}_{j}} B(z, \cdot), \quad \omega_{j}^{*}=\mathcal{B}_{j}, \quad \omega_{j}^{* \perp}=\frac{1}{2 \mathrm{i} \pi} \mathcal{A}_{j} .
$$

- Residues $t_{p, 0} \longrightarrow \omega_{p, 0}=$ third kind differential

$$
\omega_{p, 0}(z)=\mathrm{d} S_{p, o}(z)=\int_{o}^{p} B(z, \cdot), \quad \omega_{p, 0}^{*}=[o, p], \quad \omega_{p, 0}^{* \perp}=\frac{1}{2 \mathrm{i} \pi} C_{p},
$$

where $o$ is an arbitrary basepoint on $\mathcal{C}$, and $C_{p}$ is a small circle surrounding $p$ with index 1 . As we mentioned, the $t_{p, 0}$ are not independent variables, and only $\left(t_{p, 0}-t_{p_{0}, 0}\right)_{p \neq p_{0}}$ for a fixed $p_{0}$ are independent. As a consequence, we see that only differences $\omega_{p, 0}-\omega_{p^{\prime}, 0}$ and $\omega_{p, 0}^{*}-\omega_{p^{\prime}, 0}^{*}$ are independent of a choice of basepoint $o$.

- Higher times $t_{p, j}$ with $j \geq 1 \longrightarrow \omega_{p, j}=$ second kind differential

$$
\begin{aligned}
& \omega_{p, j}(z)=B_{p, j}(z)=\operatorname{Res}_{z^{\prime} \rightarrow p} \xi_{p}\left(z^{\prime}\right)^{-j} B\left(z^{\prime}, z\right), \\
& \omega_{p, j}^{*}=\frac{1}{2 \mathrm{i} \pi} \xi_{p}^{-j} C_{p}, \quad \omega_{p, j}^{* \perp}=\frac{1}{2 \mathrm{i} \pi} \xi_{p}^{j+1} C_{p} .
\end{aligned}
$$

Any meromorphic form $\omega$ is a linear combination of those basis meromorphic forms, and almost by definition we have

$$
\omega(z)=\sum_{k} t_{k} \omega_{k}(z)=\sum_{i=1}^{\mathfrak{g}} 2 \mathrm{i} \pi \epsilon_{i} \mathrm{~d} u_{i}(z)+\sum_{p \in \overline{\mathcal{P}}} t_{p, 0} \mathrm{~d} S_{p, o}(z)+\sum_{p \in \overline{\mathcal{P}}, j \geq 1} t_{p, j} B_{p, j}(z) .
$$

\section{$2.3 \quad F_{0}$}

The fact that $\int_{\omega_{i}^{*}} \int_{\omega_{j}^{*}} B\left(z, z^{\prime}\right)$ is symmetric, implies that there exists a function $F_{0}(\vec{t})$ such that

$$
\frac{\partial F_{0}}{\partial t_{i}} "=" \int_{\omega_{i}^{*}} \omega, \quad \frac{\partial^{2} F_{0}}{\partial t_{i} \partial t_{j}} "=" \int_{\omega_{i}^{*}} \int_{\omega_{j}^{*}} B
$$


The problem (this is why we write quotation marks) is that those integrals are not well-defined for times associated to 3rd kind differentials. Such a statement is correct after an appropriate regularization. When $z$ is in the vicinity of a pole $p$, we define

$$
V_{p}(z)=-\sum_{j \geq 1} \frac{t_{p, j}}{j} \xi_{p}(z)^{-j}, \quad \mathrm{~d} V_{p}(z)=\sum_{j \geq 1} t_{p, j} \frac{\mathrm{d} \xi_{p}(z)}{\xi_{p}(z)^{j+1}} .
$$

It is such that $\omega-\mathrm{d} V_{p}$ has at most a simple pole at $p$. Given an arbitrary base point $o \in \mathcal{C}$, the following integral is well-defined

$$
\mu_{p}=\int_{o}^{\infty_{p}}\left(\omega(z)-\mathrm{d} V_{p}(z)-t_{p, 0} \frac{\mathrm{d} \xi_{p}(z)}{\xi_{p}(z)}\right)-V_{p}(o)-t_{p, 0} \ln \xi_{p}(o) .
$$

$\mu_{p}$ depends on the base point $o$, but only by an additive constant independent of $p$. Since $\sum_{p} t_{p, 0}=0$, the sum $\sum_{p} t_{p, 0} \mu_{p}$ is thus independent of $o$. In some sense, $\mu_{p}$ is a regularized version of $\int_{\omega_{p, 0}^{*}} \omega$ (which does not exists). Since for all the other cycles, $\int_{\omega_{k}^{*}} \omega$ is well-defined, we can now define $F_{0}$

\section{Definition 2.6.}

$$
F_{0}(\omega)=\frac{1}{2}\left[\sum_{p \in \overline{\mathcal{P}}} \operatorname{Res}_{p} V_{p} \omega+\sum_{p \in \overline{\mathcal{P}}} t_{p, 0} \mu_{p}+\sum_{i=1}^{\mathfrak{g}} \epsilon_{i} \oint_{\mathcal{B}_{i}} \omega\right] .
$$

This definition is closely related to that of [65] where $F_{0}$ appears as a function of the times $t_{p, j}$ 's, but here we prefer to define it as a functional of a 1-form $\omega$.

Theorem 2.1 (see e.g. [65]). The first derivatives of $F_{0}$ are given by, for $j \geq 1$,

$$
\frac{\partial F_{0}}{\partial t_{p, j}}=\oint_{\omega_{p, j}^{*}} \omega=\operatorname{Res}_{p} \xi_{p}^{-j} \omega, \quad \frac{\partial F_{0}}{\partial t_{p, 0}}-\frac{\partial F_{0}}{\partial t_{p^{\prime}, 0}}=\mu_{p}-\mu_{p^{\prime}}, \quad \frac{\partial F_{0}}{\partial \epsilon_{i}}=\oint_{\mathcal{B}_{i}} \omega .
$$

The proof of this theorem has appeared in many works and contexts, initiated in [30] and generalized in [65]. In the context of Hurwitz spaces, this expression of $F_{0}$ specialized to $\omega=$ the primary differential defining the Frobenius structure, coincides with the prepotential [30, Equation (5.64)]. It follows form Theorem 2.1 that

$$
F_{0}=\frac{1}{2} \sum_{k} t_{k} \frac{\partial F_{0}}{\partial t_{k}}
$$

which means that $F_{0}$ is homogeneous of degree 2 . Another classical result is

Theorem 2.2 (see e.g. [65]). The second derivatives of $F_{0}$ are given by

$$
\frac{\partial^{2} F_{0}}{\partial t_{k} \partial t_{l}}=\int_{\omega_{k}^{*}} \int_{\omega_{l}^{*}} B
$$

except for the following cases

$$
\begin{aligned}
& \frac{\partial}{\partial t_{k}}\left(\frac{\partial}{\partial t_{p, 0}}-\frac{\partial}{\partial t_{p^{\prime}, 0}}\right) F_{0}=\int_{\omega_{k}^{*}} \int_{\omega_{p, 0}^{*}} B-\int_{\omega_{k}^{*}} \int_{\omega_{p^{\prime}, 0}^{*}} B \\
& \left(\frac{\partial}{\partial t_{p, 0}}-\frac{\partial}{\partial t_{p^{\prime}, 0}}\right)^{2} F_{0}=-\ln \left(E\left(p, p^{\prime}\right)^{2} \mathrm{~d} \xi_{p}(p) \mathrm{d} \xi_{p^{\prime}}\left(p^{\prime}\right)\right) \\
& \left(\frac{\partial}{\partial t_{p, 0}}-\frac{\partial}{\partial t_{p^{\prime}, 0}}\right)\left(\frac{\partial}{\partial t_{p, 0}}-\frac{\partial}{\partial t_{p^{\prime \prime}, 0}}\right) F_{0}=-\ln \left(\frac{E\left(p, p^{\prime}\right) E\left(p, p^{\prime \prime}\right) \mathrm{d} \xi_{p}(p)}{E\left(p^{\prime}, p^{\prime \prime}\right)}\right) \\
& \left(\frac{\partial}{\partial t_{p, 0}}-\frac{\partial}{\partial t_{p^{\prime}, 0}}\right)\left(\frac{\partial}{\partial t_{\tilde{p}, 0}}-\frac{\partial}{\partial t_{\tilde{p}^{\prime}, 0}}\right) F_{0}=-\ln \left(\frac{E\left(p, \tilde{p}^{\prime}\right) E\left(p^{\prime}, \tilde{p}\right)}{E(p, \tilde{p}) E\left(p^{\prime}, \tilde{p}^{\prime}\right)}\right) .
\end{aligned}
$$


The second derivatives of $F_{0}$ do not depend on the 1-form $\omega$, and thus do not depend on the times. Thus we have

$$
F_{0}=\frac{1}{2} \sum_{k, l} t_{k} t_{l} \frac{\partial^{2} F_{0}}{\partial t_{k} \partial t_{l}}
$$

Theorem 2.3 (see e.g. [65]).

$$
\frac{\partial^{3} F_{0}}{\partial t_{k} \partial t_{l} \partial t_{m}}=\sum_{a_{i}=\text { zeroes of } \mathrm{dX}} \operatorname{Res}_{z \rightarrow a_{i}} \frac{\omega_{k}(z) \omega_{l}(z) \omega_{m}(z)}{d X(z) d Y(z)} .
$$

\section{Reconstruction formula}

We review in this section the reconstruction $[62,63]$ of a Lax matrix whose evolution preserves the spectrum, and thus of an integrable system, from the spectral curve (see also the textbook [4] and references therein). The only difference is that, we reformulate it intrinsically in terms of 1 -forms $\omega$, instead of using time coordinates $\omega=\sum_{k} t_{k} \omega_{k}$. For this purpose, instead of BakerAkhiezer functions, we prefer to use a "spinor kernel", which is a convenient special case of Baker-Akhiezer function, which turns out to be a more intrinsic object for our formulation (see also $[13,59,60])$.

\subsection{Semiclassical spinor kernel}

Given a meromorphic 1-form $\omega$, define the 1-form

$$
\chi(z ; \omega)=\omega(z)-2 \mathrm{i} \pi \sum_{i=1}^{\mathfrak{g}} \epsilon_{i} \mathrm{~d} u_{i}(z),
$$

which depends linearly on the times (and not on the filling fractions)

$$
\chi(z ; \omega)=\sum_{p \in \overline{\mathcal{P}}} t_{p, 0} \mathrm{~d} S_{p, o}(z)+\sum_{p \in \overline{\mathcal{P}}} \sum_{j \geq 1} t_{p, j} \omega_{p, j}(z) .
$$

By construction $\chi$ is normalized on $\mathcal{A}$-cycles

$$
\oint_{\mathcal{A}_{i}} \chi=0 .
$$

Then we define the vector $\zeta(\omega)=\left[\zeta_{i}(\omega)\right]_{1 \leq i \leq \mathfrak{g}}$ with coordinates

$$
\zeta_{i}(\omega)=\frac{1}{2 \mathrm{i} \pi} \oint_{\mathcal{B}_{i}} \chi=\frac{1}{2 \mathrm{i} \pi}\left(\oint_{\mathcal{B}_{i}} \omega-\sum_{j=1}^{\mathfrak{g}} \tau_{i, j} \oint_{\mathcal{A}_{i}} \omega\right),
$$

which we write for short as

$$
\zeta(\omega)=\frac{1}{2 \mathrm{i} \pi} \oint_{\mathcal{B}-\tau \mathcal{A}} \omega .
$$

It can be decomposed as

$$
\zeta(\omega)=\sum_{p \in \overline{\mathcal{P}}} \sum_{j \geq 0} t_{p, j} \mathbf{v}_{p, j}=\sum_{k=(p, j)} t_{k} \mathbf{v}_{k}, \quad \mathbf{v}_{k}=\frac{1}{2 \mathrm{i} \pi} \oint_{\mathcal{B}} \omega_{k} .
$$


The vector $\zeta(\omega)$ is a linear function of the times $t_{k}$ and is independent of the filling fractions $\epsilon_{i}$. In other words, it follows a linear motion with constant velocity $\mathbf{v}_{k}$ in the Jacobian, as a function of any of the times $t_{k}$. A well-known property [4,31,62,63] of integrable systems is that, in appropriate variables, the motion (with any of the time $t_{k}$ ) is uniform and linear. The algebraic reconstruction takes the linear evolution in the Jacobian of $\mathcal{C}$ as starting point, and produces more complicated quantities whose evolution is described by a Lax system.

Definition 3.1. We now define the semiclassical spinor kernel as the $(1 / 2,1 / 2)$ form

$$
\psi_{\mathrm{cl}}\left(z_{1}, z_{2} ; \omega\right)=\frac{\theta\left(\mathbf{u}\left(z_{1}\right)-\mathbf{u}\left(z_{2}\right)+\zeta(\omega)+\mathbf{c}\right)}{E\left(z_{1}, z_{2}\right)} \theta(\zeta(\omega)+\mathbf{c}) \mathrm{e}^{\int_{z_{2}}^{z_{1}} \chi(z ; \omega)},
$$

where $\mathbf{c}$ is a non-singular, odd characteristics.

We write a subscript $\mathrm{cl}$ to distinguish the semiclassical spinor kernel from the one proposed in the second part of the article. This kernel was also introduced, in a similar form, in [59, 60] for solving Matrix Riemann-Hilbert problems on branched coverings of $\mathbb{C P}^{1}$.

Proposition 3.1. $\psi_{\mathrm{cl}}\left(z_{1}, z_{2} ; \omega\right)$ is a globally defined spinor in $\left(z_{1}, z_{2}\right) \in \mathcal{C} \times \mathcal{C}$, i.e. it is the squareroot of a symmetric $(1,1)$-form.

- It has a simple pole at $z_{1}=z_{2}$ : in any local coordinate $\xi(z)$

$$
\psi_{\mathrm{cl}}\left(z_{1}, z_{2} ; \omega\right) \underset{z_{1} \rightarrow z_{2}}{\sim} \frac{1}{E\left(z_{1}, z_{2}\right)} \sim \frac{\sqrt{\mathrm{d} \xi\left(z_{1}\right) \mathrm{d} \xi\left(z_{2}\right)}}{\xi\left(z_{1}\right)-\xi\left(z_{2}\right)} .
$$

- It has essential singularities when $z_{1}$ (resp. $z_{2}$ ) approaches a pole of $\omega$.

Proof. The behavior at $z_{1} \rightarrow z_{2}$ is obvious, and the essential singularities at the poles of $\omega$ come from the exponential term. What we need to prove, is that $\psi_{\mathrm{cl}}\left(z_{1}, z_{2} ; \omega\right)$ is unchanged when $z_{1}$ (resp. $z_{2}$ ) goes around a non-trivial cycle. When $z_{1}$ (resp. $z_{2}$ ) goes around an $\mathcal{A}$-cycle, the vector $\mathbf{u}\left(z_{1}\right)$ (resp. $\mathbf{u}\left(z_{2}\right)$ ) is translated by an integer vector, $\theta$ is thus unchanged, and thanks to equation $(3.1), \psi_{\mathrm{cl}}$ is unchanged when $z_{1}$ (resp. $z_{2}$ ) goes around an $\mathcal{A}$-cycle. When $z_{1}$ (resp. $\left.z_{2}\right)$ goes around a $\mathcal{B}$-cycle, the vector $\mathbf{u}\left(z_{1}\right)$ (resp. $\left.\mathbf{u}\left(z_{2}\right)\right)$ is translated by a lattice vector of the form $\tau \cdot \mathbf{n}$ with $\mathbf{n} \in \mathbb{Z}^{\mathfrak{g}}$, and $\theta$ gets multiplied by a phase according to equation (2.1). Remember that the prime form $E\left(z_{1}, z_{2}\right)$ is also a $\theta$ function, and also gets a phase given by equation (2.1). $\psi_{\mathrm{cl}}$ is thus changed by

$$
\psi_{\mathrm{cl}}\left(z_{1}+\mathbf{n} \mathcal{B}, z_{2} ; \omega\right) \rightarrow \psi_{\mathrm{cl}}\left(z_{1}, z_{2} ; \omega\right) \mathrm{e}^{-2 \mathrm{i} \pi \mathbf{n} \cdot \zeta(\omega)} \mathrm{e}^{\mathbf{n} \cdot \oint_{\mathcal{B}} \chi}
$$

and because of equation (3.1), i.e. $\zeta=\frac{1}{2 \mathrm{i} \pi} \oint_{\mathcal{B}} \chi$, we see that $\psi_{\mathrm{cl}}$ is unchanged when $z_{1}$ (resp. $z_{2}$ ) goes around a $\mathcal{B}$-cycle.

\subsection{Duality equation}

Then we construct the following spinor matrix of size $d \times d$

$$
\Psi_{\mathrm{cl}}\left(x_{1}, x_{2} ; \omega\right)=\left[\psi_{\mathrm{cl}}\left(z^{i}\left(x_{1}\right), z^{j}\left(x_{2}\right) ; \omega\right)\right]_{i, j=1}^{d},
$$

where we recall that $z^{i}(x)$ are the $d$ preimages of $x$ on the curve $\mathcal{C}$, i.e. $X\left(z^{i}(x)\right)=x$, and $d=\operatorname{deg} X$. These preimages are distinct and this matrix is well-defined when $x_{1}\left(\right.$ or $\left.x_{2}\right)$ is not at a branchpoint. 
Proposition 3.2. We have the "duality" equation

$$
\Psi_{\mathrm{cl}}\left(x_{1}, x_{2} ; \omega\right) \Psi_{\mathrm{cl}}\left(x_{2}, x_{3} ; \omega\right)=\frac{\left(x_{1}-x_{3}\right) \mathrm{d} x_{2}}{\left(x_{1}-x_{2}\right)\left(x_{2}-x_{3}\right)} \Psi_{\mathrm{cl}}\left(x_{1}, x_{3} ; \omega\right) .
$$

\section{Proof.}

$$
\frac{1}{\mathrm{~d} X(z)} \psi_{\mathrm{cl}}\left(z^{i}\left(x_{1}\right), z ; \omega\right) \psi_{\mathrm{cl}}\left(z, z^{j}\left(x_{3}\right) ; \omega\right)
$$

is a meromorphic function of $z$. Indeed, the product of two $(1 / 2)$-forms is a 1 -form, and when we divide by $\mathrm{d} X$, we get a function. The essential singularities coming from the exponentials cancel in the product, so this function can only have poles, i.e. it is meromorphic. The only possible poles are at $z=z^{i}\left(x_{1}\right)$ or $z=z^{j}\left(x_{3}\right)$ or at the zeroes of $\mathrm{d} X(z)$. Then, summing over all sheets, we see that

$$
\sum_{k} \frac{\psi_{\mathrm{cl}}\left(z^{i}\left(x_{1}\right), z^{k}\left(x_{2}\right) ; \omega\right) \psi_{\mathrm{cl}}\left(z^{k}\left(x_{2}\right), z^{j}\left(x_{3}\right) ; \omega\right)}{\mathrm{d} X\left(z^{k}\left(x_{2}\right)\right)}
$$

is a symmetric sum of a meromorphic function over all sheets of $x_{2}$, therefore it is a meromorphic function of $x_{2} \in \widehat{\mathbb{C}}$, i.e. a rational function of the complex variable $x_{2}$. It remains to find its poles. $\left.1 / \mathrm{d} X\left(z^{k}\left(x_{2}\right)\right)\right)$ behaves like $O\left(x_{2}-X\left(a_{i}\right)\right)^{-1 / 2}$ at ramification points, and since a rational function of $x_{2}$ cannot have a singularity of power $-1 / 2$, this means that this rational function has no pole at branchpoints. Its only poles can then be at $x_{2}=x_{1}$ or $x_{2}=x_{3}$, and they are simple poles. The residues of the corresponding poles are easily computed and give the theorem.

Proposition 3.3. We have a refined version of the duality equation

$$
\psi_{\mathrm{cl}}\left(z_{1}, z ; \omega\right) \psi_{\mathrm{cl}}\left(z, z_{2} ; \omega\right)=-\psi_{\mathrm{cl}}\left(z_{1}, z_{2} ; \omega\right)\left(\mathrm{d} S_{z_{1}, z_{2}}(z)-2 \mathrm{i} \pi \sum_{j=1}^{\mathfrak{g}} \alpha_{j}\left(z_{1}, z_{2} ; \omega\right) \mathrm{d} u_{j}(z)\right),
$$

where

$$
\alpha_{j}\left(z_{1}, z_{2} ; \omega\right)=\frac{\theta_{u_{j}}\left(\mathbf{u}\left(z_{1}\right)-\mathbf{u}\left(z_{2}\right)+\zeta(\omega)+\mathbf{c}\right)}{\theta\left(\mathbf{u}\left(z_{1}\right)-\mathbf{u}\left(z_{2}\right)+\zeta(\omega)+\mathbf{c}\right)}-\frac{\theta_{u_{j}}(\zeta(\omega)+\mathbf{c})}{\theta(\zeta(\omega)+\mathbf{c})} .
$$

This property, can be viewed as a special case of an "addition formula" for Baker-Akhiezer functions, found in [20]. Notice that Proposition 3.2 is a corollary of Proposition 3.3. Indeed the duality equation (Proposition 3.2) can be obtained by summing the equation above on all sheets $z=z^{k}(x)$, because $\sum_{k} \mathrm{~d} u_{i}\left(z^{k}(x)\right)=0$ and

$$
\sum_{k} \mathrm{~d} S_{z_{1}, z_{2}}\left(z^{k}(x)\right)=\frac{\left(X\left(z_{1}\right)-X\left(z_{2}\right)\right) \mathrm{d} X(z)}{\left(X(z)-X\left(z_{1}\right)\right)\left(X(z)-X\left(z_{2}\right)\right)} .
$$

Proof. Notice that $\psi_{\mathrm{cl}}\left(z_{1}, z ; \omega\right) \psi_{\mathrm{cl}}\left(z, z_{2} ; \omega\right)$ is a meromorphic 1 -form in $z$, since it has no essential singularity. It has simple poles at $z=z_{1}$ and $z=z_{2}$, with residues $\mp \psi_{\mathrm{cl}}\left(z_{1}, z_{2} ; \omega\right)$, and it has no other pole. This means that $\psi_{\mathrm{cl}}\left(z_{1}, z ; \omega\right) \psi_{\mathrm{cl}}\left(z, z_{2} ; \omega\right)+\psi_{\mathrm{cl}}\left(z_{1}, z_{2} ; \omega\right) \mathrm{d} S_{z_{1}, z_{2}}(z)$ is a holomorphic 1 -form, with no poles, therefore it must be a linear combination of the $\mathrm{d} u_{i}(z)$ 's, which we choose to write

$$
\psi_{\mathrm{cl}}\left(z_{1}, z ; \omega\right) \psi_{\mathrm{cl}}\left(z, z_{2} ; \vec{t}\right)=-\psi_{\mathrm{cl}}\left(z_{1}, z_{2} ; \omega\right)\left(\mathrm{d} S_{z_{1}, z_{2}}(z)-2 \mathrm{i} \pi \sum_{j=1}^{\mathfrak{g}} \alpha_{j}\left(z_{1}, z_{2} ; \omega\right) \mathrm{d} u_{j}(z)\right) .
$$


The left hand side is a well-defined spinor of $z_{1}$ and $z_{2}$, whereas in the right hand side,

$d S_{z_{1}, z_{2}}(z)=\int_{z_{2}}^{z_{1}} B(z, \cdot)$ gets some shifts when $z_{1}$ or $z_{2}$ go around non-trivial cycles. This implies the following relations for the coefficients $\alpha_{j}\left(z_{1}, z_{2} ; \omega\right)$

$$
\begin{aligned}
& \alpha_{j}\left(z_{1}+\mathcal{A}_{k}, z_{2} ; \omega\right)=\alpha_{j}\left(z_{1}, z_{2} ; \omega\right), \quad \alpha_{j}\left(z_{1}, z_{2}+\mathcal{A}_{k} ; \omega\right)=\alpha_{i}\left(z_{1}, z_{2} ; \omega\right), \\
& \alpha_{j}\left(z_{1}+\mathcal{B}_{k}, z_{2} ; \omega\right)=\alpha_{j}\left(z_{1}, z_{2} ; \omega\right)-2 \mathrm{i} \pi \delta_{j, k}, \quad \alpha_{j}\left(z_{1}, z_{2}+\mathcal{B}_{k} ; \omega\right)=\alpha_{j}\left(z_{1}, z_{2} ; \omega\right)+2 \mathrm{i} \pi \delta_{j, k} .
\end{aligned}
$$

Moreover, we must have $\alpha_{j}\left(z_{1}, z_{1} ; \omega\right)=0$, and $\alpha_{j}\left(z_{1}, z_{2} ; \omega\right)$ may have poles when $\psi_{\mathrm{cl}}\left(z_{1}, z_{2} ; \omega\right)=0$. Apart from those poles, $\alpha_{j}\left(z_{1}, z_{2} ; \omega\right)$ has no other singularities. The following quantity has all the required properties

$$
\frac{\theta_{u_{j}}\left(\mathbf{u}\left(z_{1}\right)-\mathbf{u}\left(z_{2}\right)+\zeta+\mathbf{c}\right)}{\theta\left(\mathbf{u}\left(z_{1}\right)-\mathbf{u}\left(z_{2}\right)+\zeta+\mathbf{c}\right)}-\frac{\theta_{u_{j}}(\zeta+\mathbf{c})}{\theta(\zeta+\mathbf{c})} .
$$

So, the difference of $\alpha_{j}$ and that quantity should be a meromorphic function of $z_{1}$ and $z_{2}$ without poles, i.e. a constant, and its value is zero by looking at $z_{1}=z_{2}$.

\subsection{Link with Baker-Akhiezer functions}

\subsubsection{Baker-Akhiezer functions}

The usual formulation of integrable systems [4, Chapter 5] is obtained by specializing one of the points to $X=\infty$. In some sense, we would like to consider

$$
\psi_{\mathrm{cl} \mid i}(z) "=" \psi_{\mathrm{cl}}\left(z, \infty_{i}\right) .
$$

The problem is, that the expression in the right hand side is divergent, and thus we again need regularizations.

The definitions in this paragraph also apply to the spinor kernel constructed in Section 5.3, so we drop here the ${ }_{\mathrm{cl}}$ index. Recall that the function $X$ has degree $d$, so the point $X=\infty$ has $d$ preimages $\infty_{i}$ (counted with multiplicities) on the curve. We define

$$
\psi_{i, 0}(z)=\lim _{z_{2} \rightarrow \infty_{i}} \frac{\psi\left(z, z_{2} ; \omega\right)}{\sqrt{\mathrm{d} \xi_{\infty_{i}}\left(z_{2}\right)}} \mathrm{e}^{V_{\infty_{i}}\left(z_{2}\right)}\left(\xi_{\infty_{i}}\left(z_{2}\right)\right)^{t_{\infty_{i}, 0}}
$$

and if $d_{\infty_{i}}>1$, we define for $0 \leq j \leq\left(d_{\infty_{i}}-1\right)$

$$
\psi_{i, j}(z)=\frac{\mathrm{d}^{j}}{\mathrm{~d} \xi_{\infty_{i}}\left(z_{2}\right)^{j}}\left(\frac{\psi\left(z, z_{2} ; \omega\right)}{\sqrt{\mathrm{d} \xi_{\infty_{i}}\left(z_{2}\right)}} \mathrm{e}^{V_{\infty_{i}}\left(z_{2}\right)}\left(\xi_{\infty_{i}}\left(z_{2}\right)\right)^{t_{\infty_{i}, 0}}\right)_{z_{2}=\infty_{i}} .
$$

There are $d$ pairs $I=(i, j)$ such that $0 \leq j \leq d_{\infty_{i}}-1$, and therefore the vector $\vec{\psi}(z)=\left[\psi_{I}(z)\right]$ is a $d$-dimensional vector, and the matrix $\Psi(x ; \omega)=\left[\psi_{I}\left(z^{k}(x)\right)\right]_{I, 1 \leq k \leq d}$ is a $d \times d$ square matrix.

\subsubsection{Dual Baker-Akhiezer functions}

Similarly, we would like to define $\phi_{\mathrm{cl} \mid i}(z) "=" \psi_{\mathrm{cl}}\left(\infty_{i}, z\right)$. Thus, we define the dual BakerAkhiezer functions

$$
\phi_{i, 0}(z)=\lim _{z_{1} \rightarrow \infty_{i}} \frac{\psi\left(z_{1}, z ; \omega\right)}{\sqrt{\mathrm{d} \xi_{\infty_{i}}\left(z_{1}\right)}} \mathrm{e}^{-V_{\infty_{i}}\left(z_{1}\right)}\left(\xi_{\infty_{i}}\left(z_{1}\right)\right)^{-t_{\infty_{i}, 0}}
$$

and if $d_{\infty_{i}}>1$, we define for each $0 \leq j \leq\left(d_{\infty_{i}}-1\right)$

$$
\phi_{i, j}(z)=\frac{\mathrm{d}^{j}}{\mathrm{~d} \xi_{\infty_{i}}\left(z_{1}\right)^{j}}\left(\frac{\psi\left(z_{1}, z ; \omega\right)}{\sqrt{\mathrm{d} \xi_{\infty_{i}}\left(z_{1}\right)}} \mathrm{e}^{-V_{\infty_{i}}\left(z_{1}\right)}\left(\xi_{\infty_{i}}\left(z_{1}\right)\right)^{-t_{\infty_{i}, 0}}\right)_{z_{1}=\infty_{i}} .
$$


There are $d$ pairs $I=(i, j)$ such that $0 \leq j \leq d_{i}-1$, and therefore the vector $\vec{\phi}(z)=\left[\phi_{I}(z)\right]$ is a $d$-dimensional vector, and the matrix $\Phi(x ; \omega)=\left[\phi_{I}\left(z^{k}(x)\right)\right]_{I, 1 \leq k \leq d}$ is a $d \times d$ square matrix. From Corollary 3.3, one retrieves the well-known result that columns of $\Phi(x ; \omega)$ are eigenvectors of a Lax matrix.

\subsection{Christoffel-Darboux relations}

Proposition 3.4. The matrix

$$
A_{\mathrm{cl}}^{-1}=\frac{1}{\mathrm{~d} x} \Phi_{\mathrm{cl}}(x) \Psi_{\mathrm{cl}}^{t}(x)
$$

is invertible, and independent of $x$. The matrix $A_{\mathrm{cl}}$ is called the Christoffel-Darboux matrix. This can also be written $\Psi_{\mathrm{cl}}^{t}(x) A_{\mathrm{cl}} \Phi_{\mathrm{cl}}(x)=\mathrm{d} x \mathbf{1}_{d \times d}$.

Proof. This is an application of Proposition 3.2, up to a conjugation. Indeed

$$
\begin{aligned}
& \left(A_{\mathrm{cl}}^{-1}\right)_{(i, k),\left(i^{\prime}, k^{\prime}\right)}=\frac{\mathrm{d}^{k^{\prime}-1}}{\mathrm{~d} \xi_{\infty_{i^{\prime}}}^{k^{\prime}-1}\left(z_{1}\right)} \frac{\mathrm{d}^{k-1}}{\mathrm{~d} \xi_{\infty_{i}}^{k-1}\left(z_{2}\right)}\left[\sum_{m} \frac{\psi_{\mathrm{cl}}\left(z_{1}, z^{m}\right) \psi_{\mathrm{cl}}\left(z^{m}, z_{2}\right)}{\sqrt{\mathrm{d} \xi_{\infty_{i^{\prime}}}\left(z_{1}\right) \mathrm{d} \xi_{\infty_{i}}\left(z_{2}\right)}}\right. \\
& \left.\times \mathrm{e}^{V_{\infty_{i}}\left(z_{2}\right)-V_{\infty_{i^{\prime}}}\left(z_{1}\right)} \xi_{\infty_{i}}\left(z_{2}\right)^{t_{\infty_{i}, 0}} \xi_{\infty_{i^{\prime}}}\left(z_{1}\right)^{-t_{\infty_{i^{\prime}}, 0}}\right]_{z_{1}=\infty_{i^{\prime}}}^{z_{2}=\infty_{i}} \\
& =\frac{\mathrm{d}^{k^{\prime}-1}}{\mathrm{~d} \xi_{\infty_{i^{\prime}}}^{k^{\prime}-1}\left(z_{1}\right)} \frac{\mathrm{d}^{k-1}}{\mathrm{~d} \xi_{\infty_{i}}^{k-1}\left(z_{2}\right)}\left[\frac{\left(X\left(z_{1}\right)-X\left(z_{2}\right)\right) \psi_{\mathrm{cl}}\left(z_{1}, z_{2}\right) \mathrm{e}^{V_{\infty_{i}}\left(z_{2}\right)-V_{\infty_{i^{\prime}}}\left(z_{1}\right)}}{\left(X(z)-X\left(z_{1}\right)\right)\left(X(z)-X\left(z_{2}\right)\right)}\right. \\
& \left.\times \frac{\xi_{\infty_{i}}\left(z_{2}\right)^{t_{\infty_{i}, 0} \xi_{\infty_{i^{\prime}}}\left(z_{1}\right)^{-t_{\infty_{i^{\prime}}, 0}}}}{\sqrt{\mathrm{d} \xi_{\infty_{i^{\prime}}}\left(z_{1}\right) \mathrm{d} \xi_{\infty_{i}}\left(z_{2}\right)}}\right]_{z_{1}=\infty_{i^{\prime}}}^{z_{2}=\infty_{i}}
\end{aligned}
$$

If $i \neq i^{\prime}$, the quantity

$$
\frac{\psi_{\mathrm{cl}}\left(z_{1}, z_{2}\right) \mathrm{e}^{V_{\infty_{i}}\left(z_{2}\right)-V_{\infty_{i^{\prime}}}\left(z_{1}\right)} \xi_{\infty_{i}}\left(z_{2}\right)^{t_{\infty_{i}, 0}} \xi_{\infty_{i^{\prime}}}\left(z_{1}\right)^{-t_{\infty_{i^{\prime}}, 0}}}{\sqrt{\mathrm{d} \xi_{\infty_{i^{\prime}}}\left(z_{1}\right) \mathrm{d} \xi_{\infty_{i}}\left(z_{2}\right)}}
$$

has a well-defined limit when $z_{1} \rightarrow \infty_{i^{\prime}}$ and $z_{2} \rightarrow \infty_{i}$, and the term $\frac{1}{X(z)-X\left(z_{1}\right)}-\frac{1}{X(z)-X\left(z_{2}\right)}$ behaves like

$$
\frac{1}{X(z)-X\left(z_{1}\right)} \underset{z_{1} \rightarrow \infty_{i^{\prime}}}{\sim} \xi_{\infty_{i^{\prime}}}\left(z_{1}\right)^{d_{\infty_{i^{\prime}}}}
$$

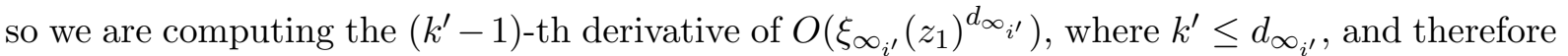
we get 0 , i.e.

$$
\left(A^{-1}\right)_{\mathrm{cl} \mid(i, k),\left(i^{\prime}, k^{\prime}\right)}=0 \quad \text { if } i \neq i^{\prime} .
$$

If $i=i^{\prime}$, we first take the limit $z_{1} \rightarrow \infty_{i}$, and again the term with $\frac{1}{X(z)-X\left(z_{1}\right)}$ vanishes. Then, remember that $\psi_{\mathrm{cl}}\left(z_{1}, z_{2}\right)$ has a simple pole at $z_{1}=z_{2}$, and thus the derivative with respect to $z_{1}$, can generate a pole of degree $k^{\prime}$ at $z_{2}=\infty_{i}$. Therefore, we are computing the $(k-1)$-th derivative of $O\left(\xi_{\infty_{i}}\left(z_{2}\right)^{d_{\infty_{i}}-k^{\prime}}\right)$. We get zero if $k+k^{\prime} \leq d_{\infty_{i}}$, and therefore

$$
\left(A^{-1}\right)_{\mathrm{cl}(i, k),\left(i, k^{\prime}\right)}=0 \quad \text { if } \quad k+k^{\prime} \leq d_{\infty_{i}} .
$$


If $i=i^{\prime}$ and $k+k^{\prime}=d_{\infty_{i}}+1$, the only non-vanishing contribution is

$$
\begin{aligned}
& \frac{1}{\left(k^{\prime}-1\right) !}\left(A^{-1}\right)_{\mathrm{cl}(i, k),\left(i, k^{\prime}\right)} \\
& =\frac{\mathrm{d}^{k-1}}{\mathrm{~d} \xi_{\infty_{i}}^{k-1}\left(z_{2}\right)} \lim _{z_{1} \rightarrow \infty_{i}}\left[\frac{E\left(z_{1}, z_{2}\right) \psi_{\mathrm{cl}}\left(z_{1}, z_{2}\right)}{\left(\xi_{\infty_{i}}\left(z_{2}\right)-\xi_{\infty_{i}}\left(z_{1}\right)\right)^{k^{\prime}}} \mathrm{e}^{V_{\infty_{i}}\left(z_{2}\right)-V_{\infty_{i}}\left(z_{1}\right)}\right. \\
& \left.\quad \quad \times \xi_{\infty_{i}}\left(z_{2}\right)^{\left.t_{\infty_{i}, 0}+d_{\infty_{i}} \xi_{\infty_{i}}\left(z_{1}\right)^{-t_{\infty_{i}, 0}}\right]}\right]_{z_{2}=\infty_{i}} \\
& =\frac{\mathrm{d}^{k-1}}{\mathrm{~d} \xi_{\infty_{i}}^{k-1}\left(z_{2}\right)} \lim _{z_{1} \rightarrow \infty_{i}}\left[\psi_{\mathrm{cl}}\left(z_{1}, z_{2}\right) E\left(z_{1}, z_{2}\right) \mathrm{e}^{V_{\infty_{i}}\left(z_{2}\right)-V_{\infty_{i}}\left(z_{1}\right)}\right. \\
& \left.\quad \times \xi_{\infty_{i}}\left(z_{2}\right)^{t_{\infty_{i}, 0}+d_{\infty_{i}}-k^{\prime}} \xi_{\infty_{i}}\left(z_{1}\right)^{-t_{\infty_{i}, 0}}\right]_{z_{2}=\infty_{i}} \\
& =\frac{\mathrm{d}^{k-1}}{\mathrm{~d} \xi_{\infty_{i}}^{k-1}\left(z_{2}\right)}\left[\xi_{\infty_{i}}\left(z_{2}\right)^{d_{\infty_{i}}-k^{\prime}}\right]_{z_{2}=\infty_{i}}=\frac{\mathrm{d}^{k-1}}{\mathrm{~d} \xi_{\infty_{i}}^{k-1}\left(z_{2}\right)}\left[\xi_{\infty_{i}}\left(z_{2}\right)^{k-1}\right]_{z_{2}=\infty_{i}}=(k-1) ! \neq 0 .
\end{aligned}
$$

The matrix $A_{\mathrm{cl}}^{-1}$ has thus typically the shape

$$
A_{\mathrm{cl}}^{-1}=\left(\begin{array}{ccccc} 
& & * & & \\
& * & * & & \\
* & * & * & & \\
& & & & * \\
& & * & *
\end{array}\right)
$$

it is made of (inverted) triangular blocks. Since the diagonal of each triangle is non-zero, this proves that the matrix $A_{\mathrm{cl}}^{-1}$ is invertible.

Then, if $i=i^{\prime}$ and $k+k^{\prime} \geq d_{\infty_{i}}+1$, we write that

$$
\frac{1}{X(z)-X\left(z_{1}\right)}=-\frac{1}{X\left(z_{1}\right)}+O\left(1 / X\left(z_{1}\right)^{2}\right)
$$

and we see that the leading term $\frac{1}{X\left(z_{1}\right)}$ is independent of $X(z)$, and the part which depends

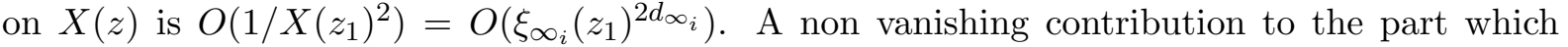
depends on $X(z)$ could occur only if $k+k^{\prime}>2 d_{\infty_{i}}$, which can never happen since we assumed $k, k^{\prime} \leq d_{\infty_{i}}$. This proves that $A_{\mathrm{cl}}$ is independent of $X(z)$.

Corollary 3.1. The matrices $\Psi_{\mathrm{cl}}(x ; \omega)$ and $\Phi_{\mathrm{cl}}(x ; \omega)$ are invertible.

As a consequence, $\psi_{\mathrm{cl}}\left(z_{1}, z_{2} ; \omega\right) / \sqrt{\mathrm{d} x\left(z_{1}\right) \mathrm{d} x\left(z_{2}\right)}$ can be identified with an integrable kernel in the sense of [49], i.e. we have

Proposition 3.5. Christoffel-Darboux relation:

$$
\psi_{\mathrm{cl}}\left(z_{1}, z_{2} ; \omega\right)=-\frac{\sum_{I, J} \psi_{\mathrm{cl} \mid I}\left(z_{1}\right) A_{\mathrm{cl} \mid I, J} \phi_{\mathrm{cl} \mid J}\left(z_{2}\right)}{X\left(z_{1}\right)-X\left(z_{2}\right)} .
$$

Proof. This is an application of Property 3.4 and Proposition 3.2. Indeed, the very definition of the $\psi_{\mathrm{cl} \mid I}$ 's, means exactly that there exists a matrix $C_{\mathrm{cl}}(x)$ such that

$$
\Psi_{\mathrm{cl}}^{t}\left(X\left(z_{1}\right)\right)=\lim _{x \rightarrow \infty} \Psi_{\mathrm{cl}}\left(X\left(z_{1}\right), x\right) C_{\mathrm{cl}}(x),
$$

and similarly, there exists a matrix $\tilde{C}_{\mathrm{cl}}(x)$ such that

$$
\Phi_{\mathrm{cl}}\left(X\left(z_{2}\right)\right)=\lim _{x \rightarrow \infty} \tilde{C}_{\mathrm{cl}}(x) \Psi_{\mathrm{cl}}\left(x, X\left(z_{2}\right)\right) .
$$


When $z_{1}=z_{2}$, we have $\Psi_{\mathrm{cl}}\left(x, X\left(z_{1}\right)\right) \Psi_{\mathrm{cl}}\left(X\left(z_{1}\right), x\right)=-\frac{\mathrm{d} x \mathrm{~d} X\left(z_{1}\right)}{\left(x-X\left(z_{1}\right)\right)^{2}} \mathbf{1}_{d \times d}$, which implies

$$
-\lim _{x \rightarrow \infty} \frac{\mathrm{d} x \mathrm{~d} X\left(z_{1}\right)}{\left(x-X\left(z_{1}\right)\right)^{2}} \tilde{C}_{\mathrm{cl}}(x) C_{\mathrm{cl}}(x)=\Phi_{\mathrm{cl}}\left(X\left(z_{1}\right)\right) \Psi_{\mathrm{cl}}^{t}\left(X\left(z_{1}\right)\right)=A_{\mathrm{cl}}^{-1} \mathrm{~d} X\left(z_{1}\right),
$$

and therefore

$$
\lim _{x \rightarrow \infty} A_{\mathrm{cl}} \tilde{C}_{\mathrm{cl}}(x) C_{\mathrm{cl}}(x) \frac{\mathrm{d} x}{x^{2}}=-\mathbf{1}_{d \times d} .
$$

Then, we have from the duality equation, for any $x$

$$
\begin{aligned}
& \left(X\left(z_{1}\right)-X\left(z_{2}\right)\right) \Psi_{\mathrm{cl}}\left(X\left(z_{1}\right), X\left(z_{2}\right)\right) \\
& \quad=\Psi_{\mathrm{cl}}\left(X\left(z_{1}\right), x\right) \Psi_{\mathrm{cl}}\left(x, X\left(z_{2}\right)\right) \frac{\left(X\left(z_{1}\right)-x\right)\left(X\left(z_{2}\right)-x\right)}{\mathrm{d} x} \\
& \quad=\Psi_{\mathrm{cl}}\left(X\left(z_{1}\right), x\right) C_{\mathrm{cl}}(x) C_{\mathrm{cl}}^{-1}(x) \Psi_{\mathrm{cl}}\left(x, X\left(z_{2}\right)\right) \frac{\left(X\left(z_{1}\right)-x\right)\left(X\left(z_{2}\right)-x\right)}{\mathrm{d} x},
\end{aligned}
$$

and in particular, we may take the limit $x \rightarrow \infty$ and insert equation (3.4)

$$
\begin{aligned}
& -\left(X\left(z_{1}\right)-X\left(z_{2}\right)\right) \Psi_{\mathrm{cl}}\left(X\left(z_{1}\right), X\left(z_{2}\right)\right) \\
& =\lim _{x \rightarrow \infty} \Psi_{\mathrm{cl}}\left(X\left(z_{1}\right), x\right) C_{\mathrm{cl}}(x) A_{\mathrm{cl}} \tilde{C}_{\mathrm{cl}}(x) C_{\mathrm{cl}}(x) C_{\mathrm{cl}}^{-1}(x) \Psi_{\mathrm{cl}}\left(x, X\left(z_{2}\right)\right) \frac{\left(X\left(z_{1}\right)-x\right)\left(X\left(z_{2}\right)-x\right)}{x^{2}} \\
& =\lim _{x \rightarrow \infty} \Psi_{\mathrm{cl}}\left(X\left(z_{1}\right), x\right) C_{\mathrm{cl}}(x) A_{\mathrm{cl}} \tilde{C}_{\mathrm{cl}}(x) \Psi_{\mathrm{cl}}\left(x, X\left(z_{2}\right)\right)=\Psi_{\mathrm{cl}}^{t}\left(X\left(z_{1}\right)\right) A_{\mathrm{cl}} \Phi_{\mathrm{cl}}\left(X\left(z_{2}\right)\right) .
\end{aligned}
$$

\subsection{Lax matrix}

As corollary of Proposition 3.2

Corollary 3.2. If $x_{1}$ and $x_{2}$ are not branchpoints, and if $x_{2} \neq x_{1}$, then the matrix $\Psi_{\mathrm{cl}}\left(x_{1}, x_{2}\right)$ is invertible, and

$$
\Psi_{\mathrm{cl}}\left(x_{1}, x_{2} ; \omega\right) \Psi_{\mathrm{cl}}\left(x_{2}, x_{1} ; \omega\right)=-\frac{\mathrm{d} x_{1} \mathrm{~d} x_{2}}{\left(x_{1}-x_{2}\right)^{2}} \mathbf{1}_{d \times d} .
$$

Proof. Take $x_{3}=x_{1}$ in the duality relation.

Corollary 3.3 (Reconstruction formula). Let

$$
\tilde{L}(x)=\operatorname{diag}\left(Y_{1}(x), \ldots, Y_{d}(x)\right)=\operatorname{diag}\left(Y\left(z^{1}(x)\right), Y\left(z^{2}(x)\right), \ldots, Y\left(z^{d}(x)\right)\right) .
$$

For every $x_{1}$, the matrix

$$
L_{x_{1}}(x ; \vec{t})=\Psi_{\mathrm{cl}}\left(x_{1}, x ; \omega\right) \tilde{L}(x) \Psi_{\mathrm{cl}}^{-1}\left(x_{1}, x ; \omega\right)=-\frac{\left(x_{1}-x\right)^{2}}{\mathrm{~d} x_{1} \mathrm{~d} x} \Psi_{\mathrm{cl}}\left(x_{1}, x ; \omega\right) \tilde{L}(x) \Psi_{\mathrm{cl}}\left(x, x_{1} ; \omega\right)
$$

(it depends on times $\vec{t}$ through $\omega=\sum_{k} t_{k} \omega_{k}$ as in equation (2.4)) is a rational function of $x$. Its characteristic polynomial is independent of the times $\vec{t}$, and its zero locus defines the semiclassical spectral curve $\operatorname{det}\left(y-L_{x_{1}}(x ; \vec{t})\right)=\operatorname{det}(y-\tilde{L}(x))=0$. Changing $x_{1}$ just amounts to a conjugation

$$
L_{x_{1}^{\prime}}(x ; \vec{t})=\Psi_{\mathrm{cl}}\left(x_{1}^{\prime}, x_{1} ; \omega\right) \tilde{L}_{x_{1}}(x ; \vec{t}) \Psi_{\mathrm{cl}}^{-1}\left(x_{1}^{\prime}, x_{1} ; \omega\right) .
$$

Proposition 3.6. For any of the times $t_{p, j}$ with $j \geq 1$, the matrix $L_{x_{1}}(x ; \vec{t})$ obeys the Lax equation

$$
\frac{\partial}{\partial t_{(p, j)}} L_{x_{1}}(x ; \vec{t})=\left[M_{(p, j) ; x_{1}}(x ; \vec{t}), L_{x_{1}}(x ; \vec{t})\right]
$$

where the matrix $M_{(p, j) ; x_{1}}$ is $M_{(p, j) ; x_{1}}(x ; \vec{t})=\partial_{t_{(p, j)}} \Psi_{\mathrm{cl}}\left(x_{1}, x ; \omega\right) \Psi_{\mathrm{cl}}^{-1}\left(x_{1}, x ; \omega\right)$. 


\subsection{Differential systems}

Baker-Akhiezer functions satisfy simultaneously several first order differential systems with respect to the spectral parameter and the times [59, 60].

Proposition 3.7. The matrix $\Psi_{\mathrm{cl}}\left(x_{1}, x ; \omega\right)$ is solution of a linear ODE with respect to the spectral parameter $x$

$$
\left(\frac{\mathrm{d}}{\mathrm{d} x}+\frac{\mathbf{1}_{d \times d}}{x-x_{1}}-M_{x ; x_{1}}(x ; \vec{t})\right) \frac{\Psi_{\mathrm{cl}}\left(x_{1}, x ; \omega\right)}{\sqrt{\mathrm{d} x \mathrm{~d} x_{1}}}=0,
$$

where $M_{x ; x_{1}}$ is a rational function of $x$ having poles only on $\overline{\mathcal{P}}$, with the same order as those of $\omega(z) / \mathrm{d} X(z)$.

Proof. Write $x=X(z)$, we have

$$
\begin{aligned}
\mathrm{d} X(z) & {\left[M_{x ; x_{1}}(X(z) ; \vec{t})\right]_{i, j}-\delta_{i, j} \frac{\mathrm{d} X(z)}{\left(X(z)-x_{1}\right)} } \\
& =-\left(X(z)-x_{1}\right)^{2} \sum_{k} \mathrm{~d}\left(\frac{\psi_{\mathrm{cl}}\left(z^{i}\left(x_{1}\right), z^{k}\right)}{\sqrt{\mathrm{d} X(z) \mathrm{d} x_{1}}}\right) \frac{\psi_{\mathrm{cl}}\left(z^{k}, z^{j}\left(x_{1}\right)\right)}{\sqrt{\mathrm{d} X(z) \mathrm{d} x_{1}}} .
\end{aligned}
$$

In the right hand side, the essential singularities cancel, and only meromorphic singularities remain. Since we perform the sum over all sheets, the result is necessarily a rational function of $X(z)$. Poles could occur at singularities of $\psi$, or also at zeroes of $\sqrt{\mathrm{d} X(z)}$, or at $X(z)=x_{1}$.

$\psi_{\mathrm{cl}}$ has a simple pole at $z^{k}=z^{i}\left(x_{1}\right)$ for some $k$. Taking the derivative yields a double pole, and multiplying by $\left(X(z)-x_{1}\right)^{2}$ cancels the double pole. If $i=j$, there is also a simple pole coming from $\psi_{\mathrm{cl}}\left(z^{k}, z^{j}\left(x_{1}\right)\right)$, and if $i \neq j$ there is no pole. It is easy to check that the residue of the pole at $x=x_{1}$ is $-\delta_{i, j}$. This implies that $M_{x ; x_{1}}(x)$ has no pole at $x=x_{1}$. From its definition, $M_{x, x_{1}}(X(z))$ must behave as $o\left((X(z)-X(a))^{-1 / 2}\right)$ at a ramification point $a$, and since it is a rational fraction of $X(z)$, this must actually be $O(1)$, meaning that $M_{x ; x_{1}}(x)$ has no poles when $x$ approaches a branchpoint.

Near $z \rightarrow p \in \overline{\mathcal{P}}$, the only singularity comes from the exponential term and we have

$$
\left[M_{x ; x_{1}}(X(z) ; \vec{t})\right]_{i, j}=\left(X(z)-x_{1}\right)^{2} \sum_{k} \omega\left(z^{k}\right) \frac{\psi_{\mathrm{cl}}\left(z^{i}\left(x_{1}\right), z^{k}\right) \psi_{\mathrm{cl}}\left(z^{k}, z^{j}\left(x_{1}\right)\right)}{(\mathrm{d} X(z))^{2} \mathrm{~d} x_{1}}+O(1) .
$$

This shows that $\mathrm{d} x M_{x ; x_{1}}(x ; \vec{t})$ has poles in $\overline{\mathcal{P}}$, of order at most that already present in $\omega / d X$.

Proposition 3.8. We have with respect to the times $t_{p, j}(j \geq 1)$

(i) $\left(\partial_{t_{p, j}}-M_{t_{p, j} ; x_{1}}(x ; \vec{t})\right) \Psi_{\mathrm{cl}}\left(x_{1}, x ; \omega\right)=0$.

(ii) $M_{t_{p, j} ; x_{1}}$ is a rational function of $x$, with possible poles only at $x=X(p)$.

If $X(p) \neq \infty$, the pole is of order $j$. If $X(p)=\infty$, the pole is of degree $1+\left\lfloor(j-1) / d_{p}\right\rfloor$.

The method of the proof is similar to that of Proposition 3.7. There also exists an analog theorem for $\partial_{t_{p, 0}}-\partial_{t_{p^{\prime}, 0}}$. We retrieve after sending $x_{1}$ to $\infty$ the usual formulation of Lax systems for $\Psi_{\mathrm{cl}}(x ; \omega)$ and $\Phi_{\mathrm{cl}}(x ; \omega)$ introduced in Section 3.3.1. 


\section{Semiclassical Tau function}

For later convenience, we define $\tilde{F}_{0}$ as a shifted version of $F_{0}$, as follows

$$
\begin{aligned}
\tilde{F}_{0}(\omega) & :=F_{0}(\omega)-\sum_{i=1}^{\mathfrak{g}} \epsilon_{i} \frac{\partial F_{0}(\omega)}{\partial \epsilon_{i}}+\mathrm{i} \pi \sum_{j, j^{\prime}=1}^{\mathfrak{g}} \epsilon_{j} \epsilon_{j^{\prime}} \tau_{j, j^{\prime}} \\
& =\frac{1}{2} \sum_{k, l=(p, j)} t_{k} t_{l} \frac{\partial^{2} F_{0}(\omega)}{\partial t_{k} t_{l}}+\frac{1}{2} \sum_{j, j^{\prime}=1}^{\mathfrak{g}} 2 \mathrm{i} \pi \epsilon_{j} \epsilon_{j^{\prime}} \tau_{j, j^{\prime}},
\end{aligned}
$$

where $\epsilon_{i}=\frac{1}{2 \mathrm{i} \pi} \oint_{\mathcal{A}_{i}} \omega$, and $\omega=\sum_{k} \omega_{k} t_{k}$.

Definition 4.1. We define

$$
\mathcal{T}_{\mathrm{cl}}(\mathcal{C}, \omega)=\mathrm{e}^{\tilde{F}_{0}(\omega)} \theta(\zeta(\omega)+\mathbf{c}),
$$

where we recall that $\zeta(\omega)=\frac{1}{2 \mathrm{i} \pi} \oint_{\mathcal{B}-\tau \mathcal{A}} \omega=\sum_{k} v_{k} t_{k}$ depends linearly on the times. We shall also sometimes use as notation $\mathcal{T}_{\mathrm{cl}}(\mathcal{C}, \omega) \equiv \mathcal{T}_{\mathrm{cl}}(\vec{t})$ when there is no ambiguity. In this article, we call $\mathcal{T}_{\mathrm{cl}}$ the "semiclassical" Tau function.

$\mathcal{T}_{\text {cl }}(\mathcal{C}, \omega)$ depends as well on the data of a non-singular odd characteristics $\mathbf{c}$ for the Theta function. It is the Tau function $[53,54,56]$ associated here to the solution of the problem of isospectral evolution described in Section 3.5. We call Tau function, any function satisfying Hirota bilinear equation, and related to Baker-Akhiezer functions by Sato relation. Sections 4.1 and 4.3 review the fact that $\mathcal{T}_{\mathrm{cl}}$ fits in this definition.

\subsection{Sato relation}

Sato relation [75] means that the Baker-Akhiezer kernel $\psi_{\mathrm{cl}}\left(z_{1}, z_{2} ; \omega\right)$ is obtained from the Tau function by a Schlesinger transformation. This can be formulated intrinsically in terms of 1 forms, as

\section{Theorem 4.1.}

$$
\frac{\mathcal{T}_{\mathrm{cl}}\left(\mathcal{C}, \omega+\mathrm{d} S_{z_{1}, z_{2}}\right)}{\mathcal{T}_{\mathrm{cl}}(\mathcal{C}, \omega)} \sqrt{\mathrm{d} X\left(z_{1}\right) \mathrm{d} X\left(z_{2}\right)}=\psi_{\mathrm{cl}}\left(z_{1}, z_{2} ; \omega\right) .
$$

Proof. We add two simple poles at $z_{1}$ and $z_{2}$ by considering

$$
\omega_{\lambda}(z)=\omega(z)+\lambda \mathrm{d} S_{z_{1}, z_{2}}(z) .
$$

According to Theorem 2.1 we have

$$
\left.\frac{\partial F_{0}}{\partial \lambda}\right|_{\lambda=0}=\mu_{z_{1}}-\mu_{z_{2}}=\int_{z_{2}}^{z_{1}} \omega
$$

and according to Theorem 2.2 we have

$$
\left.\frac{\partial^{2} F_{0}}{\partial \lambda^{2}}\right|_{\lambda=0}=-\ln \left(E\left(z_{1}, z_{2}\right)^{2} \mathrm{~d} X\left(z_{1}\right) \mathrm{d} X\left(z_{2}\right)\right) .
$$

Since $F_{0}$ is a quadratic functions of the times, and thus a degree 2 polynomial in $\lambda$, we have

$$
F_{0}\left(\omega_{\lambda}\right)=F_{0}(\omega)+\left.\lambda \frac{\partial F_{0}}{\partial \lambda}\right|_{\lambda=0}+\left.\frac{\lambda^{2}}{2} \frac{\partial^{2} F_{0}}{\partial \lambda^{2}}\right|_{\lambda=0}
$$




$$
=F_{0}(\omega)+\lambda \int_{z_{2}}^{z_{1}} \omega-\lambda^{2} \ln \left(E\left(z_{1}, z_{2}\right) \sqrt{\mathrm{d} X\left(z_{1}\right) \mathrm{d} X\left(z_{2}\right)}\right) .
$$

It is also easy to see that

$$
\zeta\left(\omega+d S_{z_{1}, z_{2}}\right)=\zeta(\omega)+\frac{\lambda}{2 \mathrm{i} \pi} \oint_{\mathcal{B}-\tau \mathcal{A}} \mathrm{d} S_{z_{1}, z_{2}}=\zeta(\omega)+\lambda\left(\mathbf{u}\left(z_{1}\right)-\mathbf{u}\left(z_{2}\right)\right) .
$$

Taking $\lambda=1$ implies the theorem.

\subsection{Expansion near poles}

We claim that Theorem 4.1 (which we wrote intrinsically in terms of 1 -forms on $\mathcal{C}$ ) is equivalent to Sato's formula (which is written in terms of times $t_{k}$ 's). In order to see that, we need to expand for $z_{1}, z_{2} \in U_{p}$ near a pole $p \in \mathcal{P}$, where $U_{p}$ denote an open neighborhood of $p$ on which the local coordinate $\xi_{p}$ is well-defined. The Schlesinger transformation

$$
\omega \rightarrow \omega+\mathrm{d} S_{z_{1}, z_{2}}
$$

can also be written by Taylor expansion, assuming ||$\xi_{p}(z) \mid>\max \left(\left|\xi_{p}\left(z_{1}\right)\right|,\left|\xi_{p}\left(z_{2}\right)\right|\right)$

$$
\mathrm{d} S_{z_{1}, z_{2}}(z)=\sum_{j \geq 1} \frac{\xi_{p}\left(z_{1}\right)^{j}}{j} \omega_{p, j}(z)-\frac{\xi_{p}\left(z_{2}\right)^{j}}{j} \omega_{p, j}(z) .
$$

Thus, the times associated to $p$ after Schlesinger transformation are

$$
\forall j \geq 1 \quad t_{p, j} \longrightarrow t_{p, j}+\left(\frac{\xi_{p}\left(z_{1}\right)^{j}}{j}-\frac{\xi_{p}\left(z_{2}\right)^{j}}{j}\right)
$$

The usual notation for this special infinite collection of times is

$$
\left[z_{1}\right]_{p}=\left(\frac{\xi_{p}\left(z_{1}\right)^{j}}{j}\right)_{j \geq 1} .
$$

Thus Theorem 4.1, together with equation (3.3) gives the Sato formula in its usual presentation involving an infinite set of times

$$
\psi_{\mathrm{cl} \mid(p, 0)}\left(z_{1}\right)=\frac{\mathcal{T}_{\mathrm{cl}}\left(\vec{t}+\left[z_{1}\right]_{p}\right)}{\mathcal{T}_{\mathrm{cl}}(\vec{t})} .
$$

\subsection{Hirota bilinear equation}

Definition 4.2. For any point $z \in \mathcal{C}$, we define the insertion operator $\delta_{z}$, which acts on functions of a meromorphic 1-form $\omega$ on $\mathcal{C}$, by considering the one parameter deformation consisting in adding a Bergman kernel $B(z, \cdot) / \mathrm{d} X(z)$ (the dot $\cdot$ means the variable on which $\omega=\omega(\cdot)$ depends)

$$
\delta_{z} f(\omega)=\left.\mathrm{d} X(z) \frac{\partial}{\partial \lambda} f\left(\omega+\lambda \frac{B(z, \cdot)}{\mathrm{d} X(z)}\right)\right|_{\lambda=0} .
$$

$\delta_{z}$ is a derivation (it satisfies the chain rule $\delta_{z}(f g)=g \delta_{z} f+f \delta_{z} g$ ). In terms of times coordinates

$$
\delta_{z} f(\vec{t})=d X(z) \partial_{t_{z, 1}} f(\vec{t})
$$


If $z$ is near a pole $p$, in the local coordinates we have

$$
\delta_{z} \equiv \sum_{j=1}^{\infty} \xi_{p}(z)^{j-1} \mathrm{~d} \xi_{p}(z) \partial_{t_{p, j}} .
$$

Again, we prefer equation (4.4), which is intrinsic and does not require the introduction of an infinite number of times. We write equation (4.5) just to make the contact with usual presentation (given for instance in [4]).

Proposition 4.1. For any two 1-forms $\omega$ and $\hat{\omega}$ defined on the same Riemann surface $\mathcal{C}$, we have

$$
\underset{z^{\prime} \rightarrow z}{\operatorname{Res}} \psi_{\mathrm{cl}}\left(z, z^{\prime} ; \omega\right) \psi_{\mathrm{cl}}\left(z^{\prime}, z ; \hat{\omega}\right)=-\delta_{z} \ln \frac{\mathcal{T}_{\mathrm{cl}}(\omega)}{\mathcal{T}_{\mathrm{cl}}(\hat{\omega})} .
$$

Proof. $\psi_{\mathrm{cl}}\left(z, z^{\prime} ; \omega\right) \psi_{\mathrm{cl}}\left(z^{\prime}, z ; \hat{\omega}\right)$ has a double pole at $z=z^{\prime}$, and thus evaluating the residue computes a derivative.

As a corollary, we get

Theorem 4.2. For any data $(\mathcal{C}, \omega)$, the Baker-Akhiezer kernel is self-replicating

$$
\delta_{z} \psi_{\mathrm{cl}}\left(z_{1}, z_{2} ; \omega\right)=-\psi_{\mathrm{cl}}\left(z_{1}, z ; \omega\right) \psi_{\mathrm{cl}}\left(z, z_{2} ; \omega\right) .
$$

Proof. We choose $\hat{\omega}=\omega+\mathrm{d} S_{z_{1}, z_{2}}$. Then, $\psi_{\mathrm{cl}}\left(z, z^{\prime} ; \omega\right) \psi_{\mathrm{cl}}\left(z^{\prime}, z ; \omega+\mathrm{d} S_{z_{1}, z_{2}}\right)$ is a meromorphic form in $z^{\prime}$ (it has no essential singularity). Its only poles are located at $z^{\prime}=z$ and $z^{\prime}=z_{2}$. Moving the integration contour, we find

$$
\begin{aligned}
\delta_{z} \ln \frac{\mathcal{T}_{\mathrm{cl}}\left(\omega+d S_{z_{1}, z_{2}}\right)}{\mathcal{T}_{\mathrm{cl}}(\omega)} & =\operatorname{Res}_{z^{\prime} \rightarrow z} \psi_{\mathrm{cl}}\left(z, z^{\prime} ; \omega\right) \psi_{\mathrm{cl}}\left(z^{\prime}, z ; \omega+\mathrm{d} S_{z_{1}, z_{2}}\right) \\
& =-\operatorname{Res}_{z^{\prime} \rightarrow z_{2}} \psi_{\mathrm{cl}}\left(z, z^{\prime} ; \omega\right) \psi_{\mathrm{cl}}\left(z^{\prime}, z ; \omega+\mathrm{d} S_{z_{1}, z_{2}}\right) .
\end{aligned}
$$

We have

$$
\begin{aligned}
\psi_{\mathrm{cl}}\left(z^{\prime}, z ; \omega+\mathrm{d} S_{z_{1}, z_{2}}\right)= & \frac{E\left(z^{\prime}, z_{1}\right) E\left(z, z_{2}\right) \mathrm{e}^{\int_{z}^{z^{\prime}} \omega+\int_{z_{2}}^{z_{1}} \omega}}{E\left(z^{\prime}, z\right) E\left(z^{\prime}, z_{2}\right) E\left(z_{1}, z\right) E\left(z_{1}, z_{2}\right)} \\
& \times \frac{\theta\left(\zeta(\omega)+\mathbf{u}\left(z^{\prime}\right)-\mathbf{u}(z)+\mathbf{u}\left(z_{1}\right)-\mathbf{u}\left(z_{2}\right)+\mathbf{c}\right)}{\theta\left(\zeta(\omega)+\mathbf{u}\left(z_{1}\right)-\mathbf{u}\left(z_{2}\right)+\mathbf{c}\right)}
\end{aligned}
$$

and thus near $z^{\prime} \rightarrow z_{2}$

$$
\begin{aligned}
\psi_{\mathrm{cl}}\left(z^{\prime}, z ; \omega+\mathrm{d} S_{z_{1}, z_{2}}\right) & \sim-\frac{1}{E\left(z^{\prime}, z_{2}\right)} \frac{\mathrm{e}^{\int_{z}^{z_{1}} \omega}}{E\left(z_{1}, z\right)} \frac{\theta\left(\zeta(\omega)+\mathbf{u}\left(z_{1}\right)-\mathbf{u}(z)+\mathbf{c}\right)}{\theta\left(\zeta(\omega)+\mathbf{u}\left(z_{1}\right)-\mathbf{u}\left(z_{2}\right)+\mathbf{c}\right)} \\
& \sim-\frac{1}{E\left(z^{\prime}, z_{2}\right)} \mathrm{e}^{\int_{z_{2}}^{z_{1}} \omega} \frac{\psi_{\mathrm{cl}}\left(z_{1}, z ; \omega\right)}{\psi_{\mathrm{cl}}\left(z_{1}, z_{2} ; \omega\right)} .
\end{aligned}
$$

Therefore

$$
\begin{aligned}
\delta_{z} \ln \frac{\mathcal{T}_{\mathrm{cl}}\left(\omega+\mathrm{d} S_{z_{1}, z_{2}}\right)}{\mathcal{T}_{\mathrm{cl}}(\omega)} & =-\operatorname{Res}_{z^{\prime} \rightarrow z_{2}} \psi_{\mathrm{cl}}\left(z, z^{\prime} ; \omega\right) \psi_{\mathrm{cl}}\left(z^{\prime}, z ; \omega+\mathrm{d} S_{z_{1}, z_{2}}\right) \\
& =-\psi_{\mathrm{cl}}\left(z_{1}, z ; \omega\right) \psi_{\mathrm{cl}}\left(z, z_{2} ; \omega\right) .
\end{aligned}
$$

This self-replication property is the analog of the Ricatti equation in [4]. Notice that it can also be obtained by a straightforward computation of $\delta_{z} \ln \psi_{\mathrm{cl}}\left(z_{1}, z_{2} ; \omega\right)$ from Definition 3.1) and comparison with the refined duality equation (Proposition 3.3). 


\section{Hirota equation in terms of times}

Theorem 4.2 is written intrinsically in terms of forms $\omega$, but to make contact with usual notations in the literature, let us translate it in terms of times $t_{k}$ 's. Given any derivation $\partial_{t}$, one defines a Hirota operator $D_{t}[47]$ acting on two functions $f(t), g(t)$, such that

$$
\left.\left(D_{t} f \cdot g\right)(t) \equiv \partial_{u} f(t+u) g(t-u)\right|_{u=0}=g^{2}(t) \partial_{t}(f / g) .
$$

In particular, there is a Hirota operator $D_{z}$ associated to derivation $\delta_{z}=\partial_{t_{z, 1}}$. This allows us to reformulate the self-replication property as

Proposition 4.2. For $\mathcal{T}_{\mathrm{cl}}=\mathcal{T}_{\mathrm{cl}}(\vec{t})$ written as a function of times, for any pole $p$, the selfreplication property is equivalent to the Hirota bilinear difference equation

$$
D_{z} \mathcal{T}_{\mathrm{cl}}\left(\vec{t}+\left[z_{1}\right]_{p}-\left[z_{2}\right]_{p}\right) \cdot \mathcal{T}_{\mathrm{cl}}(\vec{t})=-\mathcal{T}_{\mathrm{cl}}\left(\vec{t}+\left[z_{1}\right]_{p}-[z]_{p}\right) \mathcal{T}_{\mathrm{cl}}\left(\vec{t}+[z]_{p}-\left[z_{2}\right]_{p}\right) .
$$

Actually, this property for $\mathcal{T}_{\text {cl }}$ is equivalent to the Fay identity (see Section 2.1.2) satisfied by the Theta function $\theta(\cdot \mid \tau)$. The Hirota equation can also be written in a more symmetric way by setting

$$
\vec{t} \longleftarrow \vec{t}-\frac{\left[z_{1}\right]_{p}}{2}+\frac{\left[z_{2}\right]_{p}}{2}
$$

Namely

$$
\begin{aligned}
D_{z} \mathcal{T}_{\mathrm{cl}} & \left(\vec{t}+\frac{\left[z_{1}\right]_{p}}{2}-\frac{\left[z_{2}\right]_{p}}{2}\right) \cdot \mathcal{T}_{\mathrm{cl}}\left(\vec{t}-\frac{\left[z_{1}\right]_{p}}{2}+\frac{\left[z_{2}\right]_{p}}{2}\right) \\
& =-\mathcal{T}_{\mathrm{cl}}\left(\vec{t}-[z]_{p}+\frac{\left[z_{1}\right]_{p}}{2}+\frac{\left[z_{2}\right]_{p}}{2}\right) \mathcal{T}_{\mathrm{cl}}\left(\vec{t}+[z]_{p}-\frac{\left[z_{1}\right]_{p}}{2}-\frac{\left[z_{2}\right]_{p}}{2}\right) .
\end{aligned}
$$

The procedure to translate it into a set of differential equations (with respect to the times) is well-known, it is merely obtained by Taylor expansion in $\xi_{p}(z), \xi_{p}\left(z_{1}\right)$ and $\xi_{p}\left(z_{2}\right)$ (see e.g. [55]). This gives an infinite set of partial differential equations involving derivatives of the Tau function with respect to times $t_{p, j}$, which are equations of a KP hierarchy. These equations are equivalent to Hirota bilinear equation. The fact that Theta functions via Definition 4.1 provides solutions to the KP hierarchy (the so-called algebro-geometric solutions) was discovered by [52, 63]. The notion of Tau function was only introduced later in [56], but its expression for the algebrogeometric solution is a straightforward reformulation of those results, and coincides with what we call semiclassical tau function $\mathcal{T}_{\mathrm{cl}}(\vec{t})$.

\section{Proposal for a new tau function and spinor kernel}

So far, the spectral curve $(\mathcal{C}, X, Y)$ was fixed once for all, and was equipped with a 1-form $\omega$ depending linearly on times $\left(\omega=\sum_{k} t_{k} \omega_{k}\right)$. Now, we shall let the spectral curve itself change around $(\mathcal{C}, X, Y)$, and in particular we may vary the complex structure of the curve $\mathcal{C} . Y \mathrm{~d} X$ shall play the role of the 1 -form.

\subsection{More geometry: symplectic invariants}

For any spectral curve $\mathcal{S}=(\mathcal{C}, X, Y)$, a sequence of symplectic invariants $F_{g}[\mathcal{S}]$, and of symplectic covariant forms $\omega_{n}^{(g)}(\mathcal{S})$ was defined in [38]. Let us recall their definition and main properties. 


\subsubsection{Topological recursion}

Let $a_{i}$ be the ramification points of $\mathcal{S}$, i.e. the zeroes of $\mathrm{d} X$. We assume that the spectral curve $\mathcal{S}$ is regular, i.e. $a_{i}$ are simple zeroes of $\mathrm{d} X$ and $\mathrm{d} Y\left(a_{i}\right) \neq 0$. Then, $Y$ behaves like a squareroot near ramification points

$$
Y(z)=Y\left(a_{i}\right)+Y^{\prime}\left(a_{i}\right) \sqrt{X(z)-X\left(a_{i}\right)}+O\left(X(z)-X\left(a_{i}\right)\right),
$$

and there is a unique other point $\bar{z} \neq z$ such that $X(z)=X(\bar{z})$, at least for $z$ in a neighborhood $U_{a_{i}}$ of $a_{i}$. Then we define the recursion kernel [38]

$$
K\left(z_{0}, z\right)=-\frac{1}{2} \frac{\int_{\bar{z}}^{z} B\left(z_{0}, \cdot\right)}{(Y(z)-Y(\bar{z})) \mathrm{d} X(z)} .
$$

In the variable $z_{0}$, it is a meromorphic 1 -form defined globally for $z_{0} \in \mathcal{C}$, and in the variable $z$, it is the inverse of a 1-form, only defined in $\bigcup_{i} U_{a_{i}}$.

Definition 5.1. The "symplectic covariant forms" $\omega_{n}^{(g)}\left(z_{1}, \ldots, z_{n}\right)$ are meromorphic $\underbrace{(1, \ldots, 1)}_{n \text { times }}$ forms defined by the following recursion

$$
\omega_{1}^{(0)}(z)=Y(z) \mathrm{d} X(z), \quad \omega_{2}^{(0)}\left(z_{1}, z_{2}\right)=B\left(z_{1}, z_{2}\right),
$$

and for $J=\left\{z_{2}, \ldots, z_{n}\right\}$

$$
\omega_{n}^{(g)}\left(z_{0}, J\right)=\sum_{i} \operatorname{Res}_{z \rightarrow a_{i}} K\left(z_{0}, z\right)\left(\omega_{n+1}^{(g-1)}(z, \bar{z}, J)+\sum_{0 \leq h \leq g, I \subseteq J}^{\prime} \omega_{1+|I|}^{(h)}(z, I) \omega_{1+n-|I|}^{(g-h)}(\bar{z}, J \backslash I)\right),
$$

where $\sum^{\prime}$ means that terms containing a $\omega_{1}^{(0)}$ factor are excluded.

One can prove that

Theorem 5.1 ([38]). For $2-2 g-n<0, \omega_{n}^{(g)}\left(z_{1}, \ldots, z_{n}\right)$ is symmetric in its $n$ variables, and it is a meromorphic form in each variable, having poles only at ramification points, with vanishing residues. The poles are of order at most $6 g+2 n-4$.

For instance, for $(g, n)=(0,3)$, this definition yields

$$
\begin{aligned}
\omega_{3}^{(0)}\left(z_{0}, z_{1}, z_{2}\right) & =\sum_{i} \operatorname{Res}_{z \rightarrow a_{i}}\left[-\frac{1}{2} \frac{\int_{\bar{z}}^{z} B\left(z_{0}, \cdot\right)}{(Y(z)-Y(\bar{z})) \mathrm{d} X(z)}\left(B\left(z, z_{1}\right) B\left(\bar{z}, z_{2}\right)+B\left(\bar{z}, z_{1}\right) B\left(z, z_{2}\right)\right)\right] \\
& =\sum_{i} \operatorname{Res}_{z \rightarrow a_{i}} \frac{B\left(z_{0}, z\right) B\left(z_{1}, z\right) B\left(z_{2}, z\right)}{\mathrm{d} X(z) \mathrm{d} Y(z)}
\end{aligned}
$$

We shall comment this expression in Section 5.1.2 below.

Definition 5.2. The "symplectic invariants" $F_{g}$ are numbers associated to $\mathcal{S}$, as follows:

- For $g=0$, we define $F_{0}$ as in Definition 2.6, using $\omega=Y d X$, i.e., $F_{0}(\mathcal{S}):=F_{0}(Y d X)$.

- For $g=1, F_{1}$ is defined in terms of the Bergman Tau function $\mathcal{T}_{B, X}$ introduced by Kokotov and Korotkin [58]

$$
F_{1}(\mathcal{S}):=-\frac{1}{2} \ln \left(\mathcal{T}_{B, X}\right)-\frac{1}{24} \ln \left(\prod_{i} Y^{\prime}\left(a_{i}\right)\right),
$$

where $Y^{\prime}\left(a_{i}\right)=\lim _{z \rightarrow a_{i}}\left(Y(z)-Y\left(a_{i}\right)\right) / \sqrt{X(z)-X\left(a_{i}\right)} . F_{1}$ is related to the logarithm of the determinant of the Laplacian on $\mathcal{C}$ with metrics $|y \mathrm{~d} x|^{2}$. 
- For $g \geq 2$

$$
F_{g}(\mathcal{S}):=\frac{1}{2-2 g} \sum_{i} \operatorname{Res}_{z \rightarrow a_{i}} \Phi(z) \omega_{1}^{(g)}(z)
$$

where $\Phi(z)$ is any primitive $\mathrm{e}^{1}$ of $Y \mathrm{~d} X$, i.e. $\mathrm{d} \Phi=Y \mathrm{~d} X$.

As a notation we write $\omega_{0}^{(g)} \equiv F_{g}$.

The name "symplectic invariants" comes from the important property, proved in [38, 39], that $F_{g}$ and the cohomology class of $\omega_{n}^{(g)}$ are invariant under the following transformations of spectral curves, each of which let the symbolic symplectic form $\mathrm{d} X \wedge \mathrm{d} Y$ invariant

$$
(X, Y) \rightarrow(-Y, X), \quad(X, Y) \rightarrow(X, Y+R(X)), \quad(X, Y) \rightarrow(\lambda X, Y / \lambda),
$$

where $\mathrm{R}$ is any rational function and $\lambda \in \mathbb{C}^{*}$.

\subsubsection{Infinitesimal deformations: special geometry}

The times $t_{k}$ 's of the 1-form $Y \mathrm{~d} X$ provide locally a set of coordinates of the moduli space of spectral curves $\mathcal{S}=(\mathcal{C}, X, Y)$ [65]. Infinitesimal deformations of the spectral curves are given by flows associated to the 1 -forms $\omega_{k}$ dual to the times $t_{k}$ 's. The following theorem holds for the invariants $\omega_{n}^{(g)}$

Theorem $5.2([38])$. For any of the times $t_{k}$, we have

$$
\forall n, g \geq 0,\left.\quad \frac{\partial}{\partial t_{k}}\right|_{X\left(z_{i}\right) \text { fixed }} \omega_{n}^{(g)}\left(z_{1}, \ldots, z_{n}\right)=\int_{\omega_{k}^{*}} \omega_{n+1}^{(g)}\left(\cdot, z_{1}, \ldots, z_{n}\right),
$$

where $\omega_{k}^{*}$ is the dual cycle associated to the time $t_{k}$ in equation (2.2).

This set of relations is called "special geometry" by physicists in the context of string theory. Let us give some examples:

- First kind deformations/addition of holomorphic forms

$$
\frac{\partial \omega_{n}^{(g)}\left(z_{1}, \ldots, z_{n}\right)}{\partial \epsilon_{i}}=\oint_{\mathcal{B}_{i}} \omega_{n+1}^{(g)}\left(\cdot, z_{1}, \ldots, z_{n}\right)
$$

- Second kind deformations/addition of a double pole

$$
\frac{\partial \omega_{n}^{(g)}\left(z_{1}, \ldots, z_{n}\right)}{\partial t_{p, 1}}=\frac{\omega_{n+1}^{(g)}\left(p, z_{1}, \ldots, z_{n}\right)}{\mathrm{d} \xi_{p}(p)}
$$

If $p$ is a pole of $Y \mathrm{~d} X$, this does not apply to $(g, n)=(0,0)$.

- Third kind deformations/addition of simple poles

$$
\frac{\partial \omega_{n}^{(g)}\left(z_{1}, \ldots, z_{n}\right)}{\partial t_{p, 0}}-\frac{\partial \omega_{n}^{(g)}\left(z_{1}, \ldots, z_{n}\right)}{\partial t_{p^{\prime}, 0}}=\int_{p^{\prime}}^{p} \omega_{n+1}^{(g)}\left(\cdot, z_{1}, \ldots, z_{n}\right) .
$$

\footnotetext{
${ }^{1}$ Since $\omega_{1}^{(g)}(z)$ for $g \geq 2$ has no residues, $F_{g}$ is independent of the choice of $\Phi$.
} 
- $(g, n)=(0,0)$. We retrieve Theorem 2.1 (Section 2.3) for the derivatives of $F_{0}$

$$
\frac{\partial F_{0}}{\partial t_{k}}=\oint_{\omega_{k}^{*}} Y \mathrm{~d} X
$$

- $(g, n)=(0,1)$. We retrieve the definition of the form-cycle duality equation (2.3)

$$
\frac{\partial Y \mathrm{~d} X}{\partial t_{k}}(z)=\omega_{k}(z)=\oint_{\omega_{k}^{*}} B(\cdot, z) .
$$

- $(g, n)=(0,2)$. Thanks to equation $(5.1)$,

$$
\frac{\partial B\left(z_{1}, z_{2}\right)}{\partial t_{k}}=\sum_{i} \operatorname{Res}_{z \rightarrow a_{i}} \frac{B\left(z, z_{1}\right) B\left(z, z_{2}\right) \omega_{k}(z)}{\mathrm{d} X(z) \mathrm{d} Y(z)} .
$$

If we compare it to Rauch variational formula [74], we retrieve that the variation of the complex structure of $\mathcal{C}$ is such that

$$
\frac{\partial X\left(a_{i}\right)}{\partial_{t_{k}}}=\frac{\omega_{k}\left(a_{i}\right)}{\mathrm{d} Y\left(a_{i}\right)}
$$

In particular, we deduce that the time evolution of the spectral curve that we consider obeys the Whitham equations [65, 81]

$$
\left(\partial_{t_{k}} X\left(a_{i}\right)\right) \omega_{l}\left(a_{i}\right)=\left(\partial_{t_{l}} X\left(a_{i}\right)\right) \omega_{k}\left(a_{i}\right) .
$$

Furthermore, if we integrate $z_{1}$ and $z_{2}$ over the dual cycles $\omega_{l}^{*}$ and $\omega_{m}^{*}$, we retrieve Theorem 2.3 (see also [65]).

Although we make use of the meromorphic deformations (listed above) in this article, the special geometry relations may remain valid for deformations along certain multivalued 1-forms, namely the primaries and their descendents listed in [29] in the context of Hurwitz spaces, or the 1-forms coupled to the Whitham times of [65, Section 7] for a slightly different moduli space.

\subsubsection{Finite deformations}

Instead of infinitesimal deformations of the spectral curve, one may consider finite deformations: $t_{k} \rightarrow t_{k}+\frac{1}{N} c_{k}$ (where $N$ is a formal parameter, which will serve as a formal expansion parameter for Taylor series). In other words, to a spectral curve $\mathcal{S}$ and a meromorphic 1-form $\Omega=\sum_{k} c_{k} \omega_{k}$, we shall associate a new spectral curve, denoted $\mathcal{S}+\frac{1}{N} \Omega$, as push-forward of $\mathcal{S}$ by the flow defined by $\Omega$. This convenient notation should not hide the fact that the conformal structure of $\mathcal{C}$ changes under such global deformations (see equation (5.2)). A more detailed example of computations with global deformation is provided in the proof of Lemma 5.1 below.

Definition 5.3. For any meromorphic 1 -form $\Omega$ on $\mathcal{C}$, written with its times coordinates $c_{k}$ (as in equation $(2.2)$ )

$$
\Omega=\sum_{k} c_{k} \omega_{k} \quad \text { with dual cycle } \quad \Omega^{*}=\sum_{k} c_{k} \omega_{k}^{*},
$$

and for any functional $f[\mathcal{S}] \equiv f(\vec{t})$ depending on a spectral curve $\mathcal{S}$, i.e. on the times $t_{k}$ 's, we take as a definition $^{2}$ of $f\left[\mathcal{S}+\frac{1}{N} \Omega\right]$ the following formal series in $N^{-1}$

$$
f\left[\mathcal{S}+\frac{1}{N} \Omega\right]:=\sum_{k \geq 0} \frac{N^{-k}}{k !}\left(\left.\frac{\partial^{k}}{\partial \lambda^{k}} f[\vec{t}+\lambda \vec{c}]\right|_{\lambda=0}\right) .
$$

\footnotetext{
${ }^{2}$ With an appropriate regularization when $\Omega$ has simple poles.
} 
If the functional $f$ is $F_{g}$ or $\omega_{n}^{g}$, the coefficients in the Taylor series can be computed by the relations of special geometry Theorem 5.2, namely

$$
\begin{aligned}
& \omega_{n}^{(g)}\left[\mathcal{S}+\frac{1}{N} \Omega\right]\left(z_{1}, \ldots, z_{n}\right)=\omega_{n}^{(g)}[\mathcal{S}]\left(z_{1}, \ldots, z_{n}\right)+\sum_{k \geq 1} \frac{N^{-k}}{k !} \underbrace{\int_{\Omega^{*}} \ldots \int_{\Omega^{*}}}_{k \text { times }} \omega_{k+n}^{(0)}[\mathcal{S}]\left(z_{1}, \ldots, z_{n}, \bullet\right), \\
& F_{g}\left[\mathcal{S}+\frac{1}{N} \Omega\right]=F_{g}[\mathcal{S}]+\sum_{k \geq 1} \frac{N^{-k}}{k !} \underbrace{\int_{\Omega^{*}} \ldots \int_{\Omega^{*}} \omega_{k}^{(0)}[\mathcal{S}],}_{k \text { times }}
\end{aligned}
$$

so that the computation of $F_{g}$ 's or $\omega_{n}^{(g)}$ for $\mathcal{S}+\frac{1}{N} \Omega$ does not involve derivatives with respect to times, but only integrals at fixed times on $\mathcal{S}$.

\subsection{Tau function}

\subsubsection{Preliminaries}

We need Theta functions, and we shall introduce an appropriate notation for our purposes. For any $\mathbf{w} \in \mathbb{C}^{\mathfrak{g}}$, for any $\epsilon \in \mathbb{C}^{\mathfrak{g}}$, and for any $\mathfrak{g} \times \mathfrak{g}$ symmetric matrix $\tau$ of positive imaginary part $\operatorname{Im} \tau>0$, and for any $(\mu, \nu) \in \mathbb{C}^{\mathfrak{g}} \times \mathbb{C}^{\mathfrak{g}}$ we define the $\Theta_{[\mu, \nu]}$ function as

$$
\Theta_{[\mu, \nu]}(\mathbf{w} \mid \tau ; \epsilon):=\sum_{\mathbf{p} \in \mathbb{Z}^{\mathfrak{g}}} e^{\mathrm{i} \pi(\mathbf{p}+\mu-N \epsilon) \cdot \tau \cdot(\mathbf{p}+\mu-N \epsilon)+(\mathbf{p}+\mu-N \epsilon) \cdot \mathbf{w}+2 \mathrm{i} \pi \mathbf{p} \cdot \nu} .
$$

It is closely related to the usual Siegel theta function with characteristics $[\mu, \nu]$

$$
\Theta_{[\mu, \nu]}(\mathbf{w} \mid \tau ; \epsilon)=e^{\mathrm{i} \pi N^{2} \epsilon \cdot \tau \cdot \epsilon-N \epsilon \cdot \mathbf{w}-2 \mathrm{i} \pi \mu \cdot \nu} \vartheta\left[\begin{array}{l}
\mu \\
\nu
\end{array}\right](\mathbf{w} / 2 \mathrm{i} \pi-N \tau \cdot \epsilon \mid \tau),
$$

where

$$
\vartheta\left[\begin{array}{l}
\mu \\
\nu
\end{array}\right](\mathbf{w} \mid \tau)=\sum_{\mathbf{p} \in \mathbb{Z}^{\mathfrak{g}}} \exp [\mathrm{i} \pi(\mathbf{p}+\mu) \tau(\mathbf{p}+\mu)+2 \mathrm{i} \pi(\mathbf{p}+\mu)(\mathbf{w}+\nu)],
$$

$[\mu, \nu]$ plays the role of a characteristics, although we do not require it to be half-integer here. Most often, we shall omit to write the dependance in $[\mu, \nu]$, as well as the dependence in $\tau, \epsilon$, and we shall use the notation $\Theta^{\prime}, \Theta^{\prime \prime}, \ldots, \Theta^{(k)}$ for the tensor of derivatives with respect to $\mathbf{w}$. For instance

$$
\Theta^{\prime}=\left(\frac{\partial \Theta}{\partial w_{1}}, \ldots, \frac{\partial \Theta}{\partial w_{\mathfrak{g}}}\right)^{t}
$$

This $\Theta_{[\mu, \nu]}$ function satisfies the heat equation

$$
\frac{1}{2}\left(\partial_{\tau_{i, j}}+\partial_{\tau_{j, i}}\right) \Theta_{[\mu, \nu]}(\mathbf{w})=\mathrm{i} \pi \Theta^{\prime \prime}(\mathbf{w})_{i, j},
$$

where, in this equation, $\tau_{i, j}$ and $\tau_{j, i}$ are considered independent.

\subsubsection{Definition and comments}

Definition 5.4 ([35, 37]). For any $[\mu, \nu] \in \mathbb{C}^{\mathfrak{g}} \times \mathbb{C}^{\mathfrak{g}}$ and any spectral curve $\mathcal{S}=(\mathcal{C}, X, Y)$ of genus $\mathfrak{g}$, we define

$$
\mathcal{T}_{[\mu, \nu]}[\mathcal{S}]=\exp \left(\sum_{g \geq 0} N^{2-2 g} F_{g}\right)\left\{\sum_{k \geq 0} \sum_{l_{i}>0} \sum_{h_{i}>1-l_{i} / 2} \frac{N^{\sum_{i}\left(2-2 h_{i}-l_{i}\right)}}{k ! l_{1} ! \cdots l_{k} !} F_{h_{1}}^{\left(l_{1}\right)} \cdots F_{h_{k}}^{\left(l_{k}\right)} \Theta^{\left(\sum_{i} l_{i}\right)}\right\},
$$


where we defined

$$
\begin{aligned}
& F_{h}^{(k)}=\overbrace{\oint_{\mathcal{B}} \ldots \oint_{\mathcal{B}}}^{k \text { times }} \omega_{k}^{(h)}(\mathcal{S}), \quad \Theta^{(k)}=\nabla_{\mathbf{w}}^{\otimes k} \Theta_{[\mu, \nu]}\left(\mathbf{w}=\mathbf{w}_{0} \mid \tau ; \epsilon\right), \\
& \epsilon=\frac{1}{2 \mathrm{i} \pi} \oint_{\mathcal{A}} \omega_{1}^{(0)}, \quad \mathbf{w}_{0}=N F_{0}^{\prime}=N \oint_{\mathcal{B}} \omega_{1}^{(0)}, \quad \tau=\frac{1}{2 \mathrm{i} \pi} F_{0}^{\prime \prime}=\frac{1}{2 \mathrm{i} \pi} \oint_{\mathcal{B}} \oint_{\mathcal{B}} \omega_{2}^{(0)} .
\end{aligned}
$$

$\mathcal{T}$ is defined formally order by order in $N$ (in the coefficient of $1 / N^{k}$, each term $\Theta^{(j)}$ is considered formally of order 1). It can be seen as a genuine asymptotic series when $\mathcal{S}$ is such that $\mathbf{w}_{0}$ is of order $O(1)$.

To the first few orders in $1 / N$ we have explicitly

$$
\begin{aligned}
\mathcal{T}_{[\mu, \nu]}[\mathcal{S}]= & \mathrm{e}^{N^{2} F_{0}} \mathrm{e}^{F_{1}}\left\{\Theta+\frac{1}{N}\left(\Theta^{\prime} F_{1}^{\prime}+\frac{1}{6} \Theta^{\prime \prime \prime} F_{0}^{\prime \prime \prime}\right)+\frac{1}{N^{2}}\left(\Theta F_{2}+\frac{1}{2} \Theta^{\prime \prime} F_{1}^{\prime \prime}\right.\right. \\
& \left.\left.+\frac{1}{2} \Theta^{\prime \prime} F_{1}^{\prime 2}+\frac{1}{24} \Theta^{(4)} F_{0}^{\prime \prime \prime \prime}+\frac{1}{6} \Theta^{(4)} F_{0}^{\prime \prime \prime} F_{1}^{\prime}+\frac{1}{72} \Theta^{(6)} F_{0}^{\prime \prime \prime 2}\right)+o\left(1 / N^{2}\right)\right\} .
\end{aligned}
$$

Let us emphasize that this definition of $\mathcal{T}$ does not need require the computation of derivatives with respect to times, all the terms consist in cycle integrals on $\mathcal{C}$ at a given time.

Notice that when $\mu+\tau \nu$ is a non-singular half-integer odd characteristics, the leading term when $N \rightarrow \infty$ of $\mathcal{T}(\mathcal{S})$ coincides with the semiclassical Tau function of Section 4 computed for the differential form $\omega=N Y \mathrm{~d} X$, up to an exponential factor

$$
\mathcal{T}[\mathcal{S}] \sim e^{N^{2} F_{0}} \Theta\left(\mathbf{w}_{0} \mid \tau\right) \sim e^{N^{2} \tilde{F}_{0}} \theta(\zeta+\nu+\mu \cdot \tau) e^{2 \mathrm{i} \pi \mu \cdot \zeta} \sim \mathcal{T}_{\mathrm{cl}}(N Y \mathrm{~d} X) e^{2 \mathrm{i} \pi \mu \cdot \zeta} .
$$

Notice that the Fay identity for $\theta$ presented in Section 2.1 .2 is also true if we multiply $\theta$ by an exponential factor of its argument. Therefore, Theorem 4.2 ensures that the large $N$ limit of $\mathcal{T}[\mathcal{S}]$ satisfies the Hirota bilinear equation (Theorem. 4.2), i.e. is a Tau function to leading order. This is also true for arbitrary characteristics $[\mu, \nu]$, although the times have to be shifted by a (maybe complex) constant in this case. We conjecture (see Section 7 ) that $\mathcal{T}$ is actually a Tau function, i.e. satisfies Hirota equations to all orders.

We also mention that under a modular transformation (i.e. a change of choice for the $\mathcal{A}$ and $\mathcal{B}$ cycles), $\mathcal{T}$ changes like the Siegel Theta function of characteristics $[\mu, \nu]$ (see [37])

Proposition 5.1 ([37]). Under a modular $\operatorname{Sp}(2 \mathfrak{g}, \mathbb{Z})$ transformation $\tau \rightarrow \tilde{\tau}=(\alpha \tau+\beta)(\gamma \tau+\delta)^{-1}$, the characteristics $[\mu, \nu]$ changes as $\mu \rightarrow \tilde{\mu}=\delta \mu-\gamma \nu+\frac{1}{2}\left(\gamma \delta^{t}\right)_{\operatorname{diag}}, \nu \rightarrow \tilde{\nu}=-\beta \mu+\alpha \nu+\frac{1}{2}\left(\alpha \beta^{t}\right)_{\operatorname{diag}}$, the Tau function $\mathcal{T} \equiv \mathcal{T}_{[\mu, \nu]}$ transforms as

$$
\mathcal{T}_{[\mu, \nu]} \rightarrow \zeta_{[\mu, \nu]}(\alpha, \beta, \gamma, \delta) \mathcal{T}_{[\tilde{\mu}, \tilde{\nu}]},
$$

where $\zeta_{[\mu, \nu]}(\alpha, \beta, \gamma, \delta)$ is the phase factor, independent of the spectral curve.

\subsubsection{Heuristic motivation for the definition}

Let us consider

$$
Z_{N}[\mathcal{S}, \mathbf{n}]=\exp \left(\sum_{g=0}^{\infty} N^{2-2 g} F_{g}(\mathcal{S}, \mathbf{n})\right),
$$

where we emphasize the dependence of $F_{g}(\mathcal{S})=F_{g}(\mathcal{S}, \mathbf{n})$ in the vector $\mathbf{n}=\left(n_{1}, \ldots, n_{\mathfrak{g}}\right)$ of filling fractions $n_{k}=\frac{1}{2 \mathrm{i} \pi} \oint_{\mathcal{A}_{k}} Y \mathrm{~d} X$. Let $F_{g}(\mathcal{S}, \mathbf{n})$ be the symplectic invariants associated to the curve $\mathcal{S}$ 
whose vector of filling fractions is $\mathbf{n}=\left(n_{1}, \ldots, n_{\mathfrak{g}}\right) \in \mathbb{C}^{\mathfrak{g}} . \ln Z[\mathcal{S}, \mathbf{n}]$ is a formal Laurent series in $N$ (in particular we emphasize that it contains no oscillatory terms, by opposition with the definition to come). The relevance of $Z[\mathcal{S}, \mathbf{n}]$ in topological strings has been pointed out in the work of Dijkgraaf and Vafa [27]. Definition 5.4 gives a precise meaning to the sum over all shifts of filling fractions by integers

$$
\mathcal{T}_{\mu, \nu}[\mathcal{S}] "=" \sum_{\mathbf{n}^{\prime} \in \mathbb{Z}^{\mathfrak{g}}} e^{2 \mathrm{i} \pi \mathbf{n}^{\prime} \cdot \nu} Z\left[\mathcal{S}^{\prime}, \mathbf{n}+\mathbf{n}^{\prime}+\mu\right]
$$

Such sums over a lattice have also been considered in the context of the small dispersion limit of $\mathrm{KdV}$ in the genus $\mathfrak{g}$ regime [80]. In the context of hermitian matrix integrals, it has been used in $[17,35]$ to arrive to the formal asymptotic series of Definition 5.4. This series has then been proposed to describe non-perturbative effects in topological strings [37].

\subsection{Baker-Akhiezer spinor kernel}

We now define the spinor kernel $\psi\left(z_{1}, z_{2} ; \mathcal{S}\right)$ through Sato's relation

\section{Definition 5.5.}

$$
\psi\left(z_{1}, z_{2} ; \mathcal{S}\right)=\frac{\mathcal{T}\left(\mathcal{S} ; \mathcal{S}+\frac{1}{N} \mathrm{~d} S_{z_{1}, z_{2}}\right)}{\mathcal{T}(\mathcal{S})} \sqrt{\mathrm{d} X\left(z_{1}\right) \mathrm{d} X\left(z_{2}\right)}=\frac{\mathcal{T}\left(\mathcal{S}+\left[z_{1}\right]-\left[z_{2}\right]\right)}{\mathcal{T}(\mathcal{S})} \sqrt{\mathrm{d} X\left(z_{1}\right) \mathrm{d} X\left(z_{2}\right)}
$$

where $\left[z_{1}\right]-\left[z_{2}\right]=\frac{1}{N} d S_{z_{1}, z_{2}}$ is Sato's notation, see equation (4.3).

$\psi\left(z_{1}, z_{2}\right)$ is again defined formally, order by order in $1 / N$. The leading order coincides with $\psi_{\mathrm{cl}}$ introduced in Definition 3.1. Let us give the first few orders

$$
\begin{aligned}
\psi\left(z_{1}, z_{2} ; \mathcal{S}\right)= & \frac{e^{N \int_{z_{2}}^{z_{1}} Y \mathrm{~d} X}}{E\left(z_{1}, z_{2}\right)} \frac{\Theta\left(\mathbf{w}_{0}+2 \mathrm{i} \pi\left(\mathbf{u}\left(z_{1}\right)-\mathbf{u}\left(z_{2}\right)\right)\right)}{\Theta\left(\mathbf{w}_{0}\right)}\left\{1+\frac{1}{N}\left[\frac{1}{6} \int_{z_{2}}^{z_{1}} \int_{z_{2}}^{z_{1}} \int_{z_{2}}^{z_{1}} \omega_{3}^{(0)}\right.\right. \\
& +\int_{z_{2}}^{z_{1}} \omega_{1}^{(1)}+\frac{1}{2} \frac{\Theta^{\prime}\left(\mathbf{w}_{0}+2 \mathrm{i} \pi\left(\mathbf{u}\left(z_{1}\right)-\mathbf{u}\left(z_{2}\right)\right)\right)}{\Theta\left(\mathbf{w}_{0}+2 \mathrm{i} \pi\left(\mathbf{u}\left(z_{1}\right)-\mathbf{u}\left(z_{2}\right)\right)\right)} \int_{z_{2}}^{z_{1}} \int_{z_{2}}^{z_{1}} \oint_{\mathcal{B}} \omega_{3}^{(0)} \\
& +\frac{1}{2} \frac{\Theta^{\prime \prime}\left(\mathbf{w}_{0}+2 \mathrm{i} \pi\left(\mathbf{u}\left(z_{1}\right)-\mathbf{u}\left(z_{2}\right)\right)\right)}{\Theta\left(\mathbf{w}_{0}+2 \mathrm{i} \pi\left(\mathbf{u}\left(z_{1}\right)-\mathbf{u}\left(z_{2}\right)\right)\right)} \int_{z_{2}}^{z_{1}} \oint_{\mathcal{B}} \oint_{\mathcal{B}} \omega_{3}^{(0)} \\
& +\frac{1}{6}\left(\frac{\Theta^{\prime \prime \prime}\left(\mathbf{w}_{0}+2 \mathrm{i} \pi\left(\mathbf{u}\left(z_{1}\right)-\mathbf{u}\left(z_{2}\right)\right)\right)}{\Theta\left(\mathbf{w}_{0}+2 \mathrm{i} \pi\left(\mathbf{u}\left(z_{1}\right)-\mathbf{u}\left(z_{2}\right)\right)\right)}-\frac{\Theta^{\prime \prime \prime}\left(\mathbf{w}_{0}\right)}{\Theta\left(\mathbf{w}_{0}\right)}\right) F_{0}^{\prime \prime \prime} \\
& \left.\left.+\left(\frac{\Theta^{\prime}\left(\mathbf{w}_{0}+2 \mathrm{i} \pi\left(\mathbf{u}\left(z_{1}\right)-\mathbf{u}\left(z_{2}\right)\right)\right)}{\Theta\left(\mathbf{w}_{0}+2 \mathrm{i} \pi\left(\mathbf{u}\left(z_{1}\right)-\mathbf{u}\left(z_{2}\right)\right)\right)}-\frac{\Theta^{\prime}\left(\mathbf{w}_{0}\right)}{\Theta\left(\mathbf{w}_{0}\right)}\right) F_{1}^{\prime}\right]+o(1 / N)\right\} .
\end{aligned}
$$

Lemma 5.1. $\psi\left(z_{1}, z_{2} ; \mathcal{S}\right)$ is a well-defined spinor in $z_{1}$ and $z_{2}$. Furthermore

- $\psi\left(z_{1}, z_{2}\right)$ has a simple pole at $z_{1}=z_{2}$

$$
\psi\left(z_{1}, z_{2} ; \mathcal{S}\right) \underset{z_{1} \rightarrow z_{2}}{\sim} \frac{\sqrt{\mathrm{d} X\left(z_{1}\right) \mathrm{d} X\left(z_{2}\right)}}{X\left(z_{1}\right)-X\left(z_{2}\right)} .
$$

- It has an essential singularity near any pole $p$ of $Y \mathrm{~d} X$, of the form $\mathrm{e}^{N \int_{z_{2}}^{z_{1}} Y \mathrm{~d} X}$.

- At all orders in $1 / N$ (except at leading order), $\psi\left(z_{1}, z_{2}\right)$ has poles at the ramification points $a_{i}$. Their order increase with the order of $1 / N$. 
Proof. When $z_{1}$ goes around an $\mathcal{A}$-cycle, $\mathrm{d} S_{z_{1}, z_{2}}$ is unchanged. When $z_{1}$ goes around a cycle $\mathcal{B}_{j}$, $\mathrm{d} S_{z_{1}, z_{2}}$ is shifted by a holomorphic form $\mathrm{d} S_{z_{1}, z_{2}} \rightarrow \mathrm{d} S_{z_{1}, z_{2}}+2 \mathrm{i} \pi \mathrm{d} u_{j}$, which is dual to a $\partial / \partial \epsilon_{j}$, and since the Tau function is background independent (it was proved in [37] that $\partial \mathcal{T} / \partial \epsilon_{i}=0$ ), then it is unchanged. This shows that $\psi\left(z_{1}, z_{2} ; \mathcal{S}\right)$ is a well-defined spinor.

Then we compute each term of $\mathcal{T}\left(\mathcal{S}+\left[z_{1}\right]-\left[z_{2}\right]\right)$ by writing the Taylor expansion (Section 5.1.3)

$$
F_{g}\left[\mathcal{S}+\frac{\lambda}{N} \mathrm{~d} S_{z_{1}, z_{2}}\right]=\left.\sum_{n \geq 0} \frac{\lambda^{n}}{n ! N^{n}} \frac{\partial^{n} F_{g}}{\partial \lambda^{n}}\right|_{\lambda=0},
$$

which we need to evaluate at $\lambda=1$. The $n$-th derivatives of $F_{g}$ at $\lambda=0$ are computed by the special geometry relations Theorem 5.2, using the dual cycle $\left(\mathrm{d} S_{z_{1}, z_{2}}\right)^{*}=\left[z_{2}, z_{1}\right]$

$$
\left.\frac{\partial^{n} F_{g}}{\partial \lambda^{n}}\right|_{\lambda=0}=\int_{z_{2}}^{z_{1}} \cdots \int_{z_{2}}^{z_{1}} \omega_{n}^{(g)}
$$

All the $\omega_{n}^{(g)}$ with $2-2 g-n<0$ are meromorphic, and have poles only at ramification points, without residues. This implies that their contribution to $\psi\left(z_{1}, z_{2}\right)$ provides only poles at ramification points. The only terms involving $\omega_{n}^{(g)}$ with $2-2 g-n \geq 0$, are $\partial_{\lambda} F_{0}, \partial_{\lambda}^{2} F_{0}$ and $\partial_{\lambda} F_{0}^{\prime}$.

- $\left.\frac{\partial F_{0}}{\partial \lambda}\right|_{\lambda=0}=\int_{z_{2}}^{z_{1}} Y \mathrm{~d} X$, which contributes to $\psi$ as the essential singularity $\mathrm{e}^{N \int_{z_{2}}^{z_{1}} Y \mathrm{~d} X}$.

- $\left.\frac{\partial^{2} F_{0}}{\partial \lambda^{2}}\right|_{\lambda=0}=-\ln \left(E\left(z_{1}, z_{2}\right)^{2} \mathrm{~d} X\left(z_{1}\right) \mathrm{d} X\left(z_{2}\right)\right)$ which contributes to $\psi$ as $1 / E\left(z_{1}, z_{2}\right)$.

- $\left.\frac{\partial F_{0}^{\prime}}{\partial \lambda}\right|_{\lambda=0}=2 \mathrm{i} \pi\left(\mathbf{u}\left(z_{1}\right)-\mathbf{u}\left(z_{2}\right)\right)$ which does not yield any singularity.

All the other terms have $2-2 g-n<0$, and contribute order by order, only as combinations of meromorphic forms and derivatives of Theta functions, having poles at ramification points conveyed by the $\omega_{n}^{(g)}$ 's with $2-2 g-n<0$.

Notice that as a corollary of Proposition 5.1, $\psi$ has nice modular properties:

Corollary 5.1. Under a modular $\operatorname{Sp}(2 \mathfrak{g}, \mathbb{Z})$ transformation with the notations of Proposition 5.1, the spinor kernel $\psi_{[\mu, \nu]}\left(z_{1}, z_{2}\right)$ transforms as

$$
\psi_{[\mu, \nu]}\left(z_{1}, z_{2}\right) \rightarrow \psi_{[\tilde{\mu}, \tilde{\nu}]}\left(z_{1}, z_{2}\right) .
$$

\section{Correlators}

\subsection{Second kind deformations of $\mathcal{S}$}

Let us recall the definition of the insertion operator $\delta_{z}$, already encountered in Section 4.3 and adapted now for varying spectral curves.

Definition 6.1. We define the insertion operator $\delta_{z}$, acting on a functional $f(\mathcal{S})$ of a spectral curve $\mathcal{S}$, as follows

$$
\delta_{z} f=\left.\mathrm{d} X(z) \frac{\partial}{\partial \lambda} f\left(\mathcal{S}_{\lambda}\right)\right|_{\lambda=0},
$$

where the family of spectral curves $\mathcal{S}_{\lambda}=\mathcal{S}+\lambda B(z, \cdot) / \mathrm{d} X(z)$ is such that

$$
(Y \mathrm{~d} X)_{\lambda}=Y \mathrm{~d} X+\lambda \frac{B(z, \cdot)}{\mathrm{d} X(z)} .
$$

In other words $\delta_{z}=\mathrm{d} X(z) \partial / \partial t_{z, 1}$ as in Section 4.3. 
The dual cycle of $B(z, \cdot) / \mathrm{d} X(z)$ is the contour surrounding $z$ with index 1

$$
B(z, \cdot)=\operatorname{Res}_{z^{\prime} \rightarrow z} B\left(z^{\prime}, \cdot\right) \frac{\mathrm{d} X(z)}{\left(X\left(z^{\prime}\right)-X(z)\right)} .
$$

Then, the relations of special geometry (Theorem 5.2) for $\omega_{n}^{(g)}$ imply, for any $n, g \geq 0$

$$
\delta_{z} \omega_{n}^{(g)}\left(z_{1}, \ldots, z_{n}\right)=\operatorname{Res}_{z^{\prime} \rightarrow z} \omega_{n+1}^{(g)}\left(z^{\prime}, z_{1}, \ldots, z_{n}\right) \frac{\mathrm{d} X(z)}{\left(X\left(z^{\prime}\right)-X(z)\right)}=\omega_{n+1}^{(g)}\left(z, z_{1}, \ldots, z_{n}\right) .
$$

For instance

$$
\delta_{z} F_{0}=\omega_{1}^{(0)}(z)=Y(z) \mathrm{d} X(z), \quad \delta_{z} F_{g}=\omega_{1}^{(g)}(z), \quad \delta_{z} \omega_{1}^{(0)}\left(z^{\prime}\right)=B\left(z, z^{\prime}\right),
$$

and the conformal structure of $\mathcal{C}$ changes such that

$$
\delta_{z} X\left(a_{i}\right)=\frac{B\left(z, a_{i}\right)}{\mathrm{d} Y\left(a_{i}\right)} .
$$

Definition 6.2. For $n$ positive integer, we define the correlators $W_{n}\left(z_{1}, \ldots, z_{n}\right)$ and the disconnected correlators $\bar{W}_{n}\left(z_{1}, \ldots, z_{n}\right)$ as

$$
W_{n}\left(z_{1}, \ldots, z_{n}\right)=N^{-n} \delta_{z_{1}} \ldots \delta_{z_{n}} \ln \mathcal{T}(\mathcal{S}), \quad \bar{W}_{n}\left(z_{1}, \ldots, z_{n}\right)=\frac{N^{-n} \delta_{z_{1}} \cdots \delta_{z_{n}} \mathcal{T}(\mathcal{S})}{\mathcal{T}(\mathcal{S})}
$$

$W_{n}\left(z_{1}, \ldots, z_{n}\right)$ and $\bar{W}_{n}\left(z_{1}, \ldots, z_{n}\right)$ are $(1, \ldots, 1)$-forms $(n$ times $)$, symmetric in their $n$ variables. Each coefficient, order by order in $1 / N$, is a meromorphic form with poles at ramification points.

\subsection{Examples}

For instance the three first orders of $W_{1}$ are

$$
\begin{aligned}
W_{1}(z)= & N Y(z) \mathrm{d} X(z)+(\ln \Theta)^{\prime} \cdot 2 \mathrm{i} \pi \mathrm{d} \mathbf{u}(z)+\frac{1}{N}\left\{\omega_{1}^{(1)}(z)+\frac{\Theta^{\prime \prime}}{\Theta} \cdot\left(i \pi \delta_{z} \tau\right)\right. \\
& \left.+\left[F_{1}^{\prime}\left(\frac{\Theta^{\prime \prime}}{\Theta}-\frac{\Theta^{\prime 2}}{\Theta^{2}}\right)+\frac{F_{0}^{\prime \prime \prime}}{6}\left(\frac{\Theta^{\prime \prime \prime \prime}}{\Theta}-\frac{\Theta^{\prime \prime \prime} \Theta^{\prime}}{\Theta^{2}}\right)\right] \cdot 2 \mathrm{i} \pi \mathrm{d} \mathbf{u}(z)\right\}+o(1 / N),
\end{aligned}
$$

where we recall that

$$
\begin{aligned}
& \begin{aligned}
\delta_{z} \tau_{j, k} & =\frac{\delta_{z} F_{0}^{\prime \prime}}{2 \mathrm{i} \pi}=\frac{1}{2 \mathrm{i} \pi} \oint_{\mathcal{B}_{j}} \oint_{\mathcal{B}_{k}} \omega_{3}^{(0)}(\cdot, \cdot, z) \\
& =4 i \pi \sum_{l} \operatorname{Res}_{z^{\prime} \rightarrow a_{l}} K\left(z, z^{\prime}\right) \mathrm{d} u_{j}\left(z^{\prime}\right) \mathrm{d} u_{k}\left(\overline{z^{\prime}}\right)=2 \mathrm{i} \pi \sum_{l} \operatorname{Res}_{z^{\prime} \rightarrow a_{l}} \frac{B\left(z, z^{\prime}\right) \mathrm{d} u_{j}\left(z^{\prime}\right) \mathrm{d} u_{k}\left(z^{\prime}\right)}{\mathrm{d} X\left(z^{\prime}\right) \mathrm{d} Y\left(z^{\prime}\right)},
\end{aligned} \\
& \omega_{1}^{(1)}\left(z_{1}\right)=\sum_{l} \operatorname{Res}_{z \rightarrow a_{l}} K\left(z_{1}, z\right) B(z, \bar{z}) .
\end{aligned}
$$

For the 2-point correlator, the three first orders are

$$
\begin{aligned}
W_{2}\left(z_{1}, z_{2}\right)= & B\left(z_{1}, z_{2}\right)+(\ln \Theta)^{\prime \prime} \cdot 2 \mathrm{i} \pi \mathrm{d} \mathbf{u}\left(z_{1}\right) \otimes 2 \mathrm{i} \pi \mathrm{d} \mathbf{u}\left(z_{2}\right)+\frac{1}{N}\left\{\frac{\Theta^{\prime}}{\Theta} \cdot \int_{\mathcal{B}} \omega_{3}^{(0)}\left(\cdot, z_{1}, z_{2}\right)\right. \\
& +\left(\frac{\Theta^{\prime \prime}}{\Theta}\right)^{\prime} \cdot\left[i \pi\left(\delta_{z_{1}} \tau\right) \otimes 2 \mathrm{i} \pi \mathrm{d} \mathbf{u}\left(z_{2}\right)+2 \mathrm{i} \pi \mathrm{d} \mathbf{u}\left(z_{1}\right) \otimes i \pi\left(\delta_{z_{2}} \tau\right)\right]
\end{aligned}
$$




$$
\begin{aligned}
& \left.+\left[\left(F_{1}\right)^{\prime}\left(\frac{\Theta^{\prime \prime}}{\Theta}-\frac{\Theta^{\prime 2}}{\Theta^{2}}\right)^{\prime}+\frac{F_{0}^{\prime \prime \prime}}{6}\left(\frac{\Theta^{\prime \prime \prime \prime}}{\Theta}-\frac{\Theta^{\prime \prime \prime} \Theta^{\prime}}{\Theta^{2}}\right)^{\prime}\right] \cdot 2 \mathrm{i} \pi \mathrm{d} \mathbf{u}\left(z_{1}\right) \otimes 2 \mathrm{i} \pi \mathrm{d} \mathbf{u}\left(z_{2}\right)\right\} \\
& +o(1 / N) .
\end{aligned}
$$

For $n \geq 3$, the leading order of the $n$-point correlator is a $O(1)$, and is obtained by successive applications of $\delta_{z}$ to the $(\ln \Theta)^{\prime \prime}$ term

$$
\begin{aligned}
W_{n}\left(z_{1}, \ldots, z_{n}\right)= & (\ln \Theta)^{(n)} \cdot \bigotimes_{j=1}^{n} 2 \mathrm{i} \pi \mathrm{d} \mathbf{u}\left(z_{j}\right)+\frac{1}{N}\left\{\delta_{n, 3} \omega_{3}^{(0)}\left(z_{1}, z_{2}, z_{3}\right)\right. \\
& +\sum_{j=1}^{n}\left(\frac{\Theta^{\prime \prime}}{\Theta}\right)^{(n-1)} \cdot\left(i \pi \delta_{z_{j}} \tau\right) \otimes \bigotimes_{k \neq j} 2 \mathrm{i} \pi \mathrm{d} \mathbf{u}\left(z_{k}\right) \\
& +\sum_{1 \leq j<k \leq n}(\ln \Theta)^{(n-1)} \cdot\left(\int_{\mathcal{B}} \omega_{3}^{(0)}\left(\cdot, z_{j}, z_{k}\right)\right) \otimes \bigotimes_{l \neq j, k} 2 \mathrm{i} \pi \mathrm{d} \mathbf{u}\left(z_{l}\right) \\
& \left.+\left[\left(F_{1}\right)^{\prime}\left(\frac{\Theta^{\prime \prime}}{\Theta}-\frac{\Theta^{\prime 2}}{\Theta^{2}}\right)^{(n-1)}+\frac{F_{0}^{\prime \prime \prime}}{6}\left(\frac{\Theta^{\prime \prime \prime \prime}}{\Theta}-\frac{\Theta^{\prime \prime \prime} \Theta^{\prime}}{\Theta^{2}}\right)^{(n-1)}\right] \cdot \bigotimes_{j=1}^{n} 2 \mathrm{i} \pi \mathrm{d} \mathbf{u}\left(z_{j}\right)\right\} \\
& +o(1 / N) .
\end{aligned}
$$

\subsection{Loop equations}

Theorem 6.1. The dispersive Tau function obeys the loop equations. Namely, let us denote by $\Gamma$ a contour separating the poles of $Y \mathrm{~d} X$, from the set of preimages of a point $x \in \mathbb{C}$

(i) $\oint_{z \in \Gamma} \frac{\delta_{z} \ln \mathcal{T}[\mathcal{S}]-N(Y \mathrm{~d} X)(z)}{X(z)-x}=0$,

(ii) $\oint_{z \in \Gamma} \operatorname{Res}_{z^{\prime} \rightarrow z} \frac{1}{(X(z)-x)\left(X\left(z^{\prime}\right)-X(z)\right)}\left(\frac{1}{\mathcal{T}[\mathcal{S}]} \delta_{z} \delta_{z^{\prime}} \mathcal{T}[\mathcal{S}]-\frac{\mathrm{d} X(z) \mathrm{d} X\left(z^{\prime}\right)}{\left(X(z)-X\left(z^{\prime}\right)\right)^{2}}\right)=Q(x)$,

where $Q$ is a rational function of $x$, whose only poles are those of $Y \mathrm{~d} X$, and with degree one less than that of $Y \mathrm{~d} X$.

Those loop equations can be written in terms of correlators by applying $\delta_{z_{2}} \cdots \delta_{z_{n}}$.

Theorem 6.2. Let $J=\left\{z_{2}, \ldots, z_{n}\right\}$. The correlators satisfy

(i) The linear loop equations. For all $n \geq 1$

$$
\oint_{z \in \Gamma} \frac{1}{X(z)-x}\left(W_{n}(z, J)-\delta_{n, 1} N(Y \mathrm{~d} X)(z)-\delta_{n, 2} \frac{\mathrm{d} X(z) \mathrm{d} X\left(z_{2}\right)}{\left(X(z)-X\left(z_{2}\right)\right)^{2}}\right)=0 .
$$

(ii) The quadratic loop equations. For all $n \geq 1$

$$
\begin{aligned}
\oint_{z \in \Gamma} \operatorname{Res}_{z^{\prime} \rightarrow z} \frac{1}{(X(z)-x)\left(X\left(z^{\prime}\right)-X(z)\right)}\left\{\sum_{I \subseteq J} W_{1+|I|}(z, I) W_{n-|I|}\left(z^{\prime}, J \backslash I\right)\right. \\
\left.\quad+\frac{1}{N^{2}} W_{n+1}\left(z, z^{\prime}, J\right)+\mathrm{d} X(z) \mathrm{d} X\left(z^{\prime}\right) \sum_{z_{k} \in J} \mathrm{~d}_{z_{k}}\left(\frac{W_{n-1}(J)}{\left(x-X\left(z_{k}\right)\right) \mathrm{d} X\left(z_{k}\right)}\right)\right\} \\
=Q_{n}(x ; J)
\end{aligned}
$$

defines a quantity $Q_{n}(x ; J)$ which is a rational function of $x$, whose only poles are located at those of $Y \mathrm{~d} X$. 
The important information in loop equations, is that those particular combinations of $W_{n}$ 's have no monodromies in the variable $x$ around the branchpoints. Since every $W_{n}$ has poles at ramification points to all orders in $1 / N$, this is a highly non-trivial property.

Proof. The $F_{g}$ were precisely introduced such that for any $\mathbf{n}$

$$
Z[\mathcal{S}, \mathbf{n}]=\exp \left(\sum_{g} N^{2-2 g} F_{g}(\mathcal{S}, \mathbf{n}+\mu)\right)
$$

is a solution of the loop equations. Since $\tau[\mathcal{S}]$ is constructed formally as a linear combination (see equation $(5.3)$ ) of such objects, $\mathcal{T}[\mathcal{S}]$ satisfy the same loop equations. As a matter of fact, $\mathcal{T}[\mathcal{S}]$ was introduced in [35] so as to solve those loop equations while preserving modularity.

\section{Hirota equations}

We mentioned in Theorem 4.2 that the self-replication property of the Baker-Akhiezer spinor kernel $\psi_{\mathrm{cl}}$ is equivalent to an infinitesimal Fay identity for the semiclassical spectral curve, which is known in turn to be equivalent to Hirota equations. In a similar way, we conjecture here

Conjecture 7.1. $\psi$ is self-replicating

$$
\frac{1}{N}\left(\delta_{z} \psi\right)\left(z_{1}, z_{2}\right)=-\psi\left(z_{1}, z\right) \psi\left(z, z_{2}\right) .
$$

We have not been able to prove this conjecture. We prove in Appendix A that it holds up to $o(1 / N)$. We argue in Section 9 that it is compatible with what is known for spectral curves coming from the one matrix model (hyperelliptic curves), or the two matrix model. Besides, these matrix models do not allow to reach all plane curves $\mathcal{S}$. The difficulty in finding a proof of Conjecture 7.1 comes from the singularities at ramification points. For instance, one can always write

$$
\frac{1}{N}\left(\delta_{z} \psi\right)\left(z_{1}, z_{2} ; \mathcal{S}\right)=\operatorname{Res}_{z^{\prime} \rightarrow z} \psi\left(z, z^{\prime} ; \mathcal{S}\right) \psi\left(z^{\prime}, z ; \mathcal{S}+\left[z_{1}\right]-\left[z_{2}\right]\right),
$$

where $\mathcal{S}+\left[z_{1}\right]-\left[z_{2}\right]=\mathcal{S}+\frac{1}{N} \mathrm{~d} S_{z_{1}, z_{2}}$. Since the integrand is a differential form on the Riemann surface $\mathcal{C}$ underlying $\mathcal{S}$, we can move the contour to the poles at the ramification points, and the pole at $z^{\prime}=z_{2}$

$$
\frac{1}{N}\left(\delta_{z} \psi\right)\left(z_{1}, z_{2} ; \mathcal{S}\right)=-\psi\left(z_{1}, z ; \mathcal{S}\right) \psi\left(z, z_{2} ; \mathcal{S}\right)-\sum_{i} \operatorname{Res}_{z^{\prime} \rightarrow a_{i}} \psi\left(z, z^{\prime} ; \mathcal{S}\right) \psi\left(z^{\prime}, z ; \mathcal{S}+\left[z_{1}\right]-\left[z_{2}\right]\right) .
$$

Then, it remains to show that the sum of residues at ramification points vanishes. So, Conjecture 7.1 is equivalent to

\section{Conjecture 7.2.}

$$
\sum_{i} \operatorname{Res}_{z^{\prime} \rightarrow a_{i}} \psi\left(z, z^{\prime} ; \mathcal{S}\right) \psi\left(z^{\prime}, z ; \mathcal{S}+\left[z_{1}\right]-\left[z_{2}\right]\right)=0 .
$$

In Appendix A, we check that this residue at each $a_{i}$ is $o(1 / N)$. This involves already nontrivial identities between Theta functions associated to a complex curve, like Fay identity, its degenerations and differentiations with respect to the moduli of the curve, and involves the precise expression of $\omega_{3}^{(0)}$ (equation (5.1)). We have not been able yet to find a general way to show that this residue is 0 to all orders in $1 / N$.

In terms of the $\mathcal{T}$ function, Conjecture 7.1 can be rephrased 
Conjecture 7.3. $\mathcal{T}$ satisfy an infinitesimal version of Hirota equations

$$
\begin{gathered}
\mathcal{T}[\mathcal{S}]\left(\delta_{z} \mathcal{T}\right)\left[\mathcal{S}+\left[z_{1}\right]-\left[z_{2}\right]\right]-\left(\delta_{z} \mathcal{T}\right)[\mathcal{S}] \mathcal{T}\left[\mathcal{S}+\left[z_{1}\right]-\left[z_{2}\right]\right] \\
=-N \mathcal{T}\left[\mathcal{S}+\left[z_{1}\right]-[z]\right] \mathcal{T}\left[\mathcal{S}+[z]-\left[z_{2}\right]\right]
\end{gathered}
$$

There is also a global version of the former conjecture. First, notice from our definition in Section 5 that

$$
\mathcal{T}\left[\left(\mathcal{S}+\left[z_{1}\right]-\left[z_{2}\right]\right)+\left[z_{3}\right]-\left[z_{4}\right]\right]=\mathcal{T}\left[\left(\mathcal{S}+\left[z_{3}\right]-\left[z_{4}\right]\right)+\left[z_{1}\right]-\left[z_{2}\right]\right],
$$

so omitting the parentheses makes sense, but

$$
\mathcal{T}\left[\mathcal{S}+\left[z_{1}\right]-\left[z_{4}\right]+\left[z_{3}\right]-\left[z_{2}\right]\right]=-\mathcal{T}\left[\mathcal{S}+\left[z_{1}\right]-\left[z_{2}\right]+\left[z_{3}\right]-\left[z_{4}\right]\right] .
$$

This sign comes from the fact that the definition of $\mathcal{T}$ contains a regularization procedure (for $\left.\int_{z_{i}}^{z_{j}} \int_{z_{i}}^{z_{j}} B\right)$, whose result depends on the way we form the pairs of simple poles to add to $\mathcal{S}$.

Conjecture 7.4. $\mathcal{T}$ satisfies Hirota equations

$$
\begin{aligned}
& \mathcal{T}\left[\left(\mathcal{S}+\left[z_{1}\right]-\left[z_{2}\right]\right)+\left[z_{3}\right]-\left[z_{4}\right]\right] \mathcal{T}[\mathcal{S}] \\
& \quad=\mathcal{T}\left[\mathcal{S}+\left[z_{1}\right]-\left[z_{2}\right]\right] \mathcal{T}\left[\mathcal{S}+\left[z_{3}\right]-\left[z_{4}\right]\right]-\mathcal{T}\left[\mathcal{S}+\left[z_{3}\right]-\left[z_{2}\right]\right] \mathcal{T}\left[\mathcal{S}+\left[z_{1}\right]-\left[z_{4}\right]\right]
\end{aligned}
$$

Provided our conjectures hold, $\mathcal{T}$ and $\psi$ are actually the Tau function and the "wave function" of a dispersive integrable system.

Proof of equivalence of Conjectures 7.3 and 7.4. We can obtain Conjecture 7.3 from Conjecture 7.4 by letting $z_{1}$ and $z_{2}$ merge to a point $z$. In the other direction, we use shorter notations

$$
\mathcal{T}_{i j k l}=\mathcal{T}\left[\mathcal{S}+\left[z_{i}\right]-\left[z_{j}\right]+\left[z_{k}\right]-\left[z_{l}\right]\right], \quad \mathcal{T}_{i j}=\mathcal{T}\left[\mathcal{S}+\left[z_{i}\right]-\left[z_{j}\right]\right], \quad \mathcal{T}=\mathcal{T}[\mathcal{S}],
$$

and we apply Conjecture 7.3 to the spectral curve $\mathcal{S}+\left[z_{3}\right]-\left[z_{4}\right]$

$$
\delta_{z} \mathcal{T}_{1234}=\frac{\delta_{z} \mathcal{T}_{34}}{\mathcal{T}_{34}} \mathcal{T}_{1234}-N \frac{\mathcal{T}_{1 z 34} \mathcal{T}_{z 234}}{\mathcal{T}_{34}}=\frac{\delta_{z} \mathcal{T}}{\mathcal{T}} \mathcal{T}_{1234}-N \frac{\mathcal{T}_{3 z} \mathcal{T}_{z 4} \mathcal{T}_{1234}}{\mathcal{T} \mathcal{T}_{34}}-N \frac{\mathcal{T}_{1 z 34} \mathcal{T}_{z 234}}{\mathcal{T}_{34}}
$$

Exchanging the roles of $z_{1} \leftrightarrow z_{3}$ and $z_{2} \leftrightarrow z_{4}$ also gives

$$
\delta_{z} \mathcal{T}_{1234}=\frac{\delta_{z} \mathcal{T}}{\mathcal{T}} \mathcal{T}_{1234}-N \frac{\mathcal{T}_{1 z} \mathcal{T}_{z 2} \mathcal{T}_{1234}}{\mathcal{T} \mathcal{T}_{12}}-N \frac{\mathcal{T}_{3 z 12} \mathcal{T}_{z 412}}{\mathcal{T}_{12}}
$$

and comparing the two, we may get rid of the terms involving $\delta_{z}$

$$
\mathcal{T}_{12} \mathcal{T}_{3 z} \mathcal{T}_{z 4} \mathcal{T}_{1234}+\mathcal{T} \mathcal{T}_{12} \mathcal{T}_{1 z 34} \mathcal{T}_{z 234}=\mathcal{T}_{34} \mathcal{T}_{1 z} \mathcal{T}_{z 2} \mathcal{T}_{1234}+\mathcal{T} \mathcal{T}_{34} \mathcal{T}_{3 z 12} \mathcal{T}_{z 412}
$$

Let us define

$$
U_{1234}=\mathcal{T} \mathcal{T}_{1234}-\mathcal{T}_{12} \mathcal{T}_{34}+\mathcal{T}_{14} \mathcal{T}_{32}
$$

which is the quantity which should vanish at the end of our computation. Notice that

$$
\lim _{z_{1} \rightarrow z_{2}} \mathcal{U}_{1234}=0 .
$$

Indeed

$$
\mathcal{U}_{1234} \underset{z_{1} \rightarrow z_{2}}{\sim}\left(\frac{\mathcal{T} \mathcal{T}_{34}}{E_{12}}+\mathcal{T} \delta_{1} \mathcal{T}_{34}-\frac{\mathcal{T} \mathcal{T}_{34}}{E_{12}}-\delta_{1} \mathcal{T} \mathcal{T}_{34}+\mathcal{T}_{14} \mathcal{T}_{31}\right)
$$




$$
\underset{z_{1} \rightarrow z_{2}}{\sim}\left(\mathcal{T} \delta_{1} \mathcal{T}_{34}-\delta_{1} \mathcal{T} \mathcal{T}_{34}+\mathcal{T}_{14} \mathcal{T}_{31}\right)
$$

where $E_{i j}=E\left(z_{i}, z_{j}\right)$, and this expression vanish by application of Conjecture 7.3 to the spectral curve $\mathcal{S}$. Notice also that the remark about $\mathcal{T}$ made above Conjecture 7.4 implies $\mathcal{U}_{i j k l}=\mathcal{U}_{k l i j}=$ $-\mathcal{U}_{i l k j}$. Let us rewrite equation (7.1) in terms of $\mathcal{U}_{i j k l}$ only

$$
\begin{aligned}
& \mathcal{T}_{12} \mathcal{U}_{1 z 34} \mathcal{U}_{z 234}-\mathcal{T}_{34} \mathcal{U}_{3 z 12} \mathcal{U}_{z 412}+\left(\mathcal{T}_{12} \mathcal{T}_{3 z} \mathcal{T}_{z 4}-\mathcal{T}_{1 z} \mathcal{T}_{z 2} \mathcal{T}_{34}\right) \mathcal{U}_{1234} \\
& \quad+\left(\mathcal{T}_{1 z} \mathcal{T}_{34}-\mathcal{T}_{14} \mathcal{T}_{3 z}\right) \mathcal{T}_{12} \mathcal{U}_{z 234}+\left(\mathcal{T}_{z 2} \mathcal{T}_{34}-\mathcal{T}_{32} \mathcal{T}_{z 4}\right) \mathcal{T}_{12} \mathcal{U}_{1 z 34} \\
& \quad+\left(\mathcal{T}_{32} \mathcal{T}_{1 z}-\mathcal{T}_{12} \mathcal{T}_{3 z}\right) \mathcal{T}_{34} \mathcal{U}_{z 412}+\left(\mathcal{T}_{z 2} \mathcal{T}_{14}-\mathcal{T}_{12} \mathcal{T}_{z 4}\right) \mathcal{T}_{34} \mathcal{U}_{3 z 12}=0
\end{aligned}
$$

The left hand side may have simple poles when $z_{i} \rightarrow z_{j}$ due to $\mathcal{T}_{i j}$, but not higher degree poles since we know that $\mathcal{U}_{i j k l}$ is actually regular when $z_{i} \rightarrow z_{j}$ or $z_{l}$. For equation (7.2) to hold, in particular, the coefficient of the simple pole when $z_{2} \rightarrow z_{3}$ must vanish

$$
-\mathcal{T}_{13} \mathcal{T}_{z 4} \mathcal{U}_{1 z 34}+\mathcal{T}_{1 z} \mathcal{T}_{34} \mathcal{U}_{z 413}=0
$$

which we can also write after reindexing the points and using the symmetries of $\mathcal{U}$

$$
\mathcal{T}_{31} \mathcal{T}_{z 4} \mathcal{U}_{1 z 34}=\mathcal{T}_{3 z} \mathcal{T}_{14} \mathcal{U}_{z 134}
$$

Similarly, the coefficient of the simple pole when $z_{1} \rightarrow z_{2}$ must vanish

$$
\mathcal{U}_{1 z 34} \mathcal{U}_{z 134}+\left(\mathcal{T}_{z 1} \mathcal{T}_{34}-\mathcal{T}_{z 4} \mathcal{T}_{31}\right) \mathcal{U}_{1 z 34}+\left(\mathcal{T}_{1 z} \mathcal{T}_{34}-\mathcal{T}_{14} \mathcal{T}_{3 z}\right) \mathcal{U}_{z 134}=0
$$

Now, we may combine the latter with equation (7.3), set $z=z_{2}$ for convenience, and isolate $\mathcal{U}_{1234}$

$$
\frac{\mathcal{T}_{31} \mathcal{T}_{24}}{\mathcal{T}_{32} \mathcal{T}_{14}} \mathcal{U}_{1234}^{2}+\left(\left(\mathcal{T}_{21} \mathcal{T}_{34}-\mathcal{T}_{24} \mathcal{T}_{31}\right)+\frac{\mathcal{T}_{31} \mathcal{T}_{24}}{\mathcal{T}_{32} \mathcal{T}_{14}}\left(\mathcal{T}_{12} \mathcal{T}_{34}-\mathcal{T}_{14} \mathcal{T}_{32}\right)\right) \mathcal{U}_{1234}=0
$$

If $\mathcal{U}_{1234}$ were not identically zero, we would have (by continuity of all the coefficients of the series) for any points $z_{1}, z_{2}, z_{3}, z_{4}$ on the curve

$$
\mathcal{U}_{1234}=\frac{\mathcal{T}_{32} \mathcal{T}_{14}}{\mathcal{T}_{31} \mathcal{T}_{24}}\left(\mathcal{T}_{24} \mathcal{T}_{31}-\mathcal{T}_{21} \mathcal{T}_{34}\right)+\left(\mathcal{T}_{14} \mathcal{T}_{32}-\mathcal{T}_{12} \mathcal{T}_{34}\right)
$$

But matching the coefficient of the simple pole when $z_{1} \rightarrow z_{4}$ in this equation yields

$$
0=\frac{\mathcal{T}_{32}}{\mathcal{T}_{31} \mathcal{T}_{21}}\left(\mathcal{T}_{21} \mathcal{T}_{31}-\mathcal{T}_{21} \mathcal{T}_{31}\right)+\mathcal{T}_{32}=\mathcal{T}_{32}
$$

which is not true. Therefore, $\mathcal{U}_{1234} \equiv 0$.

\section{Consequences}

\subsection{Exponential formula}

We start with a remark which does not rely on the conjectures of Section 7, but which is natural to present now. Recall that the kernel is defined by Sato's formula

$$
\psi\left(z_{1}, z_{2} ; \mathcal{S}\right)=\frac{\mathcal{T}\left(\mathcal{S}+\frac{1}{N} \mathrm{~d} S_{z_{1}, z_{2}}\right)}{\mathcal{T}(\mathcal{S})} \sqrt{\mathrm{d} X\left(z_{1}\right) \mathrm{d} X\left(z_{2}\right)} .
$$

Adding a double pole can be realized by adding two simple poles and taking the limit where the two simple poles collapse. In other words, we can write

$$
\mathrm{d} S_{z_{1}, z_{2}}=\int_{z_{2}}^{z_{1}} B=\left(X\left(z_{1}\right)-X\left(z_{2}\right)\right) B\left(\cdot, z_{1}\right) / \mathrm{d} X\left(z_{1}\right)+O\left(\left(X\left(z_{1}\right)-X\left(z_{2}\right)\right)\right)^{2},
$$


and then express $\psi$ in terms of second kind deformations of $\mathcal{T}$, i.e. in terms of the correlators $W_{n}$. However, when we substitute second kind deformations instead of third kind deformations, we must pay attention to regularization for the term $\int_{z_{2}}^{z_{1}} \int_{z_{2}}^{z_{1}} B$, like in Sections 4.1 and 5.3. Besides, for any $n, W_{n}$ has a finite number of terms which are not $O(1 / N)$. For this reason we define the following quantities, which are $O(1 / N)$

$$
\begin{aligned}
& \widehat{W}_{1}(z)=W_{1}(z)-N Y \mathrm{~d} X(z)-(\ln \Theta)^{\prime} \cdot 2 \mathrm{i} \pi \mathrm{d} \mathbf{u}(z), \\
& \widehat{W}_{2}\left(z_{1}, z_{2}\right)=W_{2}\left(z_{1}, z_{2}\right)-B\left(z_{1}, z_{2}\right)-(\ln \Theta)^{\prime \prime} \cdot 2 \mathrm{i} \pi \mathrm{d} \mathbf{u}\left(z_{1}\right) \otimes 2 \mathrm{i} \pi \mathrm{d} \mathbf{u}\left(z_{2}\right),
\end{aligned}
$$

and for $n \geq 3$

$$
\widehat{W}_{n}\left(z_{1}, \ldots, z_{n}\right)=W_{n}\left(z_{1}, \ldots, z_{n}\right)-(\ln \Theta)^{(n)} \cdot \bigotimes_{j=1}^{n} 2 \mathrm{i} \pi \mathrm{d} \mathbf{u}\left(z_{j}\right) .
$$

Then, we have the exponential formula

\section{Proposition 8.1.}

$$
\psi\left(z_{1}, z_{2}\right)=\frac{e^{N \int_{z_{2}}^{z_{1}} Y \mathrm{~d} X}}{E\left(z_{1}, z_{2}\right)} \frac{\Theta_{12}}{\Theta} \exp \left(\sum_{n \geq 1} \frac{1}{n !} \int_{z_{2}}^{z_{1}} \cdots \int_{z_{2}}^{z_{1}} \widehat{W}_{n}\right),
$$

where $\Theta_{12}=\Theta\left(\mathbf{w}_{0}+2 \mathrm{i} \pi\left(\mathbf{u}\left(z_{1}\right)-\mathbf{u}\left(z_{2}\right)\right)\right)$ and $\Theta=\Theta\left(\mathbf{w}_{0}\right)$. This formula is an equality if we collect on both sides all the terms of the same order.

Proof. The Taylor formula allows to express $\ln \mathcal{T}\left[\mathcal{S}+\left[z_{1}\right]-\left[z_{2}\right]\right]-\ln \mathcal{T}[\mathcal{S}]$ to all orders in $1 / N$. For the first and second order, we use equations (4.1) and (4.2)

$$
\begin{aligned}
\ln \mathcal{T}[\mathcal{S} & \left.+\left[z_{1}\right]-\left[z_{2}\right]\right]-\ln \mathcal{T}[\mathcal{S}]=\int_{z_{2}}^{z_{1}} N^{-1} \delta_{\zeta}(\ln \mathcal{T})+\frac{1}{2} " \int_{z_{2}}^{z_{1}} \int_{z_{2}}^{z_{1}} N^{-2} \delta_{\zeta} \delta_{\zeta^{\prime}}(\ln \mathcal{T})[\mathcal{S}] " \\
& +\sum_{n \geq 3} \frac{N^{-n}}{n !} \int_{z_{2}}^{z_{1}} \underbrace{\delta_{\zeta_{1}} \cdots \int_{z_{2}}^{z_{1}} \delta_{\zeta_{n}}}_{n \text { times }}(\ln \mathcal{T})[\mathcal{S}] \\
= & \int_{z_{2}}^{z_{1}} W_{1}+\frac{1}{2} " \int_{z_{2}}^{z_{1}} \int_{z_{2}}^{z_{1}} W_{2} "+\sum_{n \geq 3} \frac{1}{n !} \underbrace{\int_{z_{2}}^{z_{1}} \cdots \int_{z_{2}}^{z_{1}} W_{n}}_{n \text { times }} \\
= & N \int_{z_{2}}^{z_{1}} Y \mathrm{~d} X-\frac{1}{2} \ln \left(\left(E\left(z_{1}, z_{2}\right)\right)^{2} \mathrm{~d} X\left(z_{1}\right) \mathrm{d} X\left(z_{2}\right)\right) \\
& +\sum_{n \geq 1} \frac{1}{n !}(\ln \Theta)^{(n)} \cdot\left(2 \mathrm{i} \pi\left(\mathbf{u}\left(z_{1}\right)-\mathbf{u}\left(z_{2}\right)\right)\right)^{\otimes n}+\sum_{n \geq 1} \frac{1}{n !} \underbrace{\int_{z_{2}}^{z_{1}} \ldots \int_{z_{2}}^{z_{1}}}_{n \text { times }} \widehat{W}_{n} .
\end{aligned}
$$

In the last step, we have used the expression for the leading order of $W_{n}$ found in Section 6.2. Then, the second line can be resummated into $\ln \Theta_{12}$, and the whole result exponentiated leads to the announced formula.

Notice that, when expanding the exponential, to any given order $O\left(N^{-k}\right)$, all the $W_{n}$ give a contribution involving derivatives of theta functions contracted with tensor products $\left(\mathbf{u}\left(z_{1}\right)-\right.$ $\left.\mathbf{u}\left(z_{2}\right)\right)^{\otimes n}$. These contributions have to be resummated into a single theta function (or derivatives thereof) with argument shifted by $\mathbf{u}\left(z_{1}\right)-\mathbf{u}\left(z_{2}\right)$, then producing an expression at the order sought involving only a finite number of terms. 


\subsection{Determinantal formulas}

Conversely, the correlators $W_{n}\left(z_{1}, \ldots, z_{n}\right)$ can be retrieved from the spinor kernel $\psi\left(z_{1}, z_{2}\right)$, they are the determinantal correlation functions built with $\psi\left(z_{i}, z_{j}\right)$. Let us consider first the case of $W_{1}$, which does not rely on the conjectures of Section 7 .

\section{Lemma 8.1.}

$$
W_{1}(z)=N Y \mathrm{~d} X(z)+\lim _{z^{\prime} \rightarrow z}\left(\psi\left(z^{\prime}, z\right) e^{-N \int_{z_{2}}^{z_{1}} Y \mathrm{~d} X}-\frac{\sqrt{\mathrm{d} X\left(z^{\prime}\right) \mathrm{d} X(z)}}{X\left(z^{\prime}\right)-X(z)}\right) .
$$

Proof. First, we notice that adding a double pole can be realized by adding two simple poles and take the limit where the two simple poles collapse. More precisely, when $z^{\prime} \rightarrow z$, we have

$$
\mathrm{d} S_{z^{\prime}, z}\left(z_{0}\right) \underset{z^{\prime} \rightarrow z}{\sim}\left(X\left(z^{\prime}\right)-X(z)\right) \frac{B\left(z, z_{0}\right)}{\mathrm{d} X(z)} .
$$

We can thus use the definition of $\delta_{z}$ with $\lambda=X\left(z^{\prime}\right)-X(z)$. For any regular functional $f[\mathcal{S}]$ of the spectral curve, we thus have

$$
f\left[\mathcal{S}+\frac{1}{N} \mathrm{~d} S_{z^{\prime}, z}\right]=f[\mathcal{S}]+\frac{X\left(z^{\prime}\right)-X(z)}{N \mathrm{~d} X(z)} \delta_{z} f[\mathcal{S}]+O\left(\left(X\left(z^{\prime}\right)-X(z)\right)^{2}\right) .
$$

In some sense, we trade a variation of $\mathcal{S}$ by a second kind differential with a variation with a third kind differential.

In particular, $F_{g}$ with $g \geq 1$, or every $\Theta$-term in Definition 5.4 are regular functionals of the spectral curve. We just have to pay attention to the $F_{0}$ term, because the derivative of $F_{0}$ with respect to third kind differentials involves a regularization procedure, whereas the derivative with respect to second kind differentials does not. We have, by Taylor expansion, and computing all derivatives from special geometry (Theorem 5.2)

$$
\begin{aligned}
F_{0}\left[\mathcal{S}+\lambda \mathrm{d} S_{z^{\prime}, z}\right]= & F_{0}[\mathcal{S}]+\left.\sum_{n=1}^{\infty} \frac{\lambda^{n}}{n !} \frac{\partial^{n}}{\partial \lambda^{n}} F_{0}\left[\mathcal{S}+\lambda \mathrm{d} S_{z^{\prime}, z}\right]\right|_{\lambda=0} \\
= & F_{0}[\mathcal{S}]+\lambda \int_{z}^{z^{\prime}} Y \mathrm{~d} X-\frac{\lambda^{2}}{2} \ln \left(E\left(z, z^{\prime}\right)^{2} \mathrm{~d} X(z) \mathrm{d} X\left(z^{\prime}\right)\right) \\
& +\sum_{n=3}^{\infty} \frac{\lambda^{n}}{n !} \int_{z}^{z^{\prime}} \cdots \int_{z}^{z^{\prime}} W_{n}^{(0)} \\
= & F_{0}(\mathcal{S})+\lambda \int_{z}^{z^{\prime}} Y \mathrm{~d} X-\frac{\lambda^{2}}{2} \ln \left(E\left(z, z^{\prime}\right)^{2} \mathrm{~d} X(z) \mathrm{d} X\left(z^{\prime}\right)\right) \\
& +O\left(\left(X\left(z^{\prime}\right)-X(z)\right)^{2}\right) .
\end{aligned}
$$

Taking $\lambda=1 / N$ gives

$$
\begin{aligned}
F_{0}[\mathcal{S} & \left.+\frac{1}{N} \mathrm{~d} S_{z^{\prime}, z}\right]+\frac{1}{N^{2}} \ln \left(E\left(z^{\prime}, z\right) \sqrt{\mathrm{d} X(z) \mathrm{d} X\left(z^{\prime}\right)}\right) \\
& =F_{0}[\mathcal{S}]+\frac{X\left(z^{\prime}\right)-X(z)}{N \mathrm{~d} X(z)} \delta_{z} F_{0}[\mathcal{S}]+O\left(\left(X\left(z^{\prime}\right)-X(z)\right)^{2}\right) .
\end{aligned}
$$

Finally we have

$$
\psi\left(z^{\prime}, z\right) \mathrm{e}^{-N \int_{z}^{z^{\prime}} Y \mathrm{~d} X} E\left(z^{\prime}, z\right)=1+\frac{X\left(z^{\prime}\right)-X(z)}{N \mathrm{~d} X(z)} \delta_{z}\left(\ln \mathcal{T}-N^{2} F_{0}\right)+O\left(\left(X\left(z^{\prime}\right)-X(z)\right)^{2}\right),
$$


and thus

$$
\psi\left(z^{\prime}, z\right) \mathrm{e}^{-N \int_{z}^{z^{\prime}} Y \mathrm{~d} X}-\frac{1}{E\left(z^{\prime}, z\right)}=\frac{X\left(z^{\prime}\right)-X(z)}{N \mathrm{~d} X(z) E\left(z^{\prime}, z\right)} \delta_{z}\left(\ln \mathcal{T}-N^{2} F_{0}\right)+O\left(X\left(z^{\prime}\right)-X(z)\right) .
$$

Taking the limit $z^{\prime} \rightarrow z$, and noticing that

$$
\frac{1}{E\left(z^{\prime}, z\right)}=\sqrt{\mathrm{d} X(z) \mathrm{d} X\left(z^{\prime}\right)} /\left(X\left(z^{\prime}\right)-X(z)\right)+O\left(X\left(z^{\prime}\right)-X(z)\right)
$$

we find

$$
\begin{aligned}
\frac{1}{N} \delta_{z}\left(\ln \mathcal{T}-N^{2} F_{0}\right) & =W_{1}(z)-N Y(z) \mathrm{d} X(z) \\
& =\lim _{z^{\prime} \rightarrow z}\left(\psi\left(z^{\prime}, z\right) \mathrm{e}^{-N \int_{z}^{z^{\prime}} Y \mathrm{~d} X}-\frac{\sqrt{\mathrm{d} X\left(z^{\prime}\right) \mathrm{d} X(z)}}{X\left(z^{\prime}\right)-X(z)}\right),
\end{aligned}
$$

hence the lemma.

Theorem 8.1. If Conjecture 7.1 holds, then

$$
\forall n \geq 2, \quad W_{n}\left(z_{1}, \ldots, z_{n}\right)=(-1)^{n+1} \sum_{\sigma \text { cyclic perm. }} \prod_{i=1}^{n} \psi\left(z_{i}, z_{\sigma(i)}\right) .
$$

Equivalently

$$
\bar{W}_{n}\left(z_{1}, \ldots, z_{n}\right)=\text { "det" } \psi\left(z_{i}, z_{j}\right),
$$

where "det" means that when we decompose the determinant as a sum of permutations, each factor $\psi\left(z_{i}, z_{i}\right)$ should be replaced by $W_{1}\left(z_{i}\right)$.

Proof. The formula for $W_{1}$ is proved in Lemma 8.1. Then, we get the formula for $W_{n}$ by recursively applying $\delta_{z_{i}}$ and using the self-replication of $\psi$.

It is thus clear that this determinantal structure relies on the (conjectured) existence of Hirota equations for $\mathcal{T}$, i.e. on integrability.

\subsection{Baker-Akhiezer functions}

We recall the notations of Section 3. With the kernel $\psi\left(z_{1}, z_{2}\right)$ that we have just constructed, we introduce a $d \times d$ matrix $\Psi\left(x_{1}, x_{2}\right)=\psi\left(z^{i}\left(x_{1}\right), z^{j}\left(x_{2}\right)\right)_{i, j=1, \ldots, d}$ for $x_{1}, x_{2} \in \mathbb{C}$, where $z^{j}(x)$ are the $d$ preimages of $x$ on the curve. This definition has to be regularized when $x_{1}$ or $x_{2}$ is equal to $X(p)$ where $p$ is a pole of $X$ or $Y$. In particular, it was explained in Section 3.3 how to take $x_{1}$ or $x_{2} \rightarrow \infty$

$$
\Psi(x) "="\left[\Psi\left(z^{j}(x), \infty_{I}\right)\right]_{I, 1 \leq j \leq d}, \quad \Phi(x) "="\left[\Psi\left(\infty_{I}, z^{j}(x)\right)\right]_{I, 1 \leq j \leq d}
$$

\subsection{Duality equation}

Theorem 8.2. If Conjecture 7.1 holds, we have for any spectral curve $\mathcal{S}$

$$
\Psi\left(x_{1}, x_{2}\right) \Psi\left(x_{2}, x_{3}\right)=\frac{\left(x_{1}-x_{3}\right) \mathrm{d} x_{2}}{\left(x_{2}-x_{1}\right)\left(x_{3}-x_{1}\right)} \Psi\left(x_{1}, x_{3}\right) .
$$


Proof. For $\mathcal{S}=(\mathcal{C}, x, y)$, we introduce an auxiliary spectral curve

$$
\hat{\mathcal{S}}_{i j}=\mathcal{S}+\frac{1}{N} \mathrm{~d} S_{z^{i}\left(x_{1}\right), z^{j}\left(x_{3}\right)} .
$$

To compute the matrix element, we use the self-replication of $\psi$

$$
\begin{aligned}
{\left[\Psi\left(x_{1}, x_{2}\right) \Psi\left(x_{2}, x_{3}\right)\right]_{i j} } & =\sum_{m} \psi\left(z^{i}\left(x_{1}\right), z^{m}\left(x_{2}\right)\right) \psi\left(z^{m}\left(x_{2}\right), z^{j}\left(x_{3}\right)\right) \\
& =-\sum_{m} \delta_{z^{m}\left(x_{2}\right)} \ln \left(\frac{\mathcal{T}\left[\hat{\mathcal{S}}_{i j}\right]}{\mathcal{T}[\mathcal{S}]}\right) .
\end{aligned}
$$

Now, we use the fact that $\delta_{z}$ is a derivation, and by definition, $\delta_{z} \ln \mathcal{T}\left[\hat{\mathcal{S}}_{i j}\right]=W_{1}\left[z ; \hat{\mathcal{S}}_{i j}\right]$

$$
\begin{aligned}
& {\left[\Psi\left(x_{1}, x_{2}\right) \Psi\left(x_{2}, x_{3}\right)\right]_{i j}} \\
& =-\sum_{m} W_{1}\left[z^{m}\left(x_{2}\right) ; \hat{\mathcal{S}}_{i j}\right] \psi\left(z^{i}\left(x_{1}\right), z^{j}\left(x_{3}\right)\right)+\sum_{m} W_{1}\left[z^{m}\left(x_{2}\right) ; \mathcal{S}\right] \psi\left(z^{i}\left(x_{1}\right), z^{j}\left(x_{3}\right)\right) .
\end{aligned}
$$

The linear loop equation (Theorem 6.1) tells us the sum over sheets of $W_{1}\left[z^{m}\left(x_{2}\right) ; \hat{\mathcal{S}}_{i j}\right]$

$$
\begin{aligned}
& {\left[\Psi\left(x_{1}, x_{2}\right) \Psi\left(x_{2}, x_{3}\right)\right]_{i j}=\left[\Psi\left(x_{1}, x_{3}\right)\right]_{i j}} \\
& \quad \times\left(-\sum_{m} N Y \mathrm{~d} X\left(z^{m}\left(x_{2}\right)\right)-\mathrm{d} S_{z^{i}\left(x_{1}\right), z^{j}\left(x_{3}\right)}\left(z^{m}\left(x_{2}\right)\right)+N Y \mathrm{~d} X\left(z^{m}\left(x_{2}\right)\right)\right) \\
& \quad=-\left[\Psi\left(x_{1}, x_{3}\right)\right]_{i j} \sum_{m} \mathrm{~d} S_{z^{i}\left(x_{1}\right), z^{j}\left(x_{3}\right)}\left(z^{m}\left(x_{2}\right)\right)=\frac{\left(x_{1}-x_{3}\right) \mathrm{d} x_{2}}{\left(x_{1}-x_{2}\right)\left(x_{2}-x_{3}\right)}\left[\Psi\left(x_{1}, x_{3}\right)\right]_{i j} .
\end{aligned}
$$

The Baker-Akhiezer spinor kernel $\psi_{\mathrm{cl}}\left(z_{1}, z_{2}\right)$ of Section 3 was regular at ramification points. So, we could find a formula for $\psi_{\mathrm{cl}}\left(z_{1}, z\right) \psi_{\mathrm{cl}}\left(z, z_{2}\right)$ even before summing over the sheets where $z$ is located (refined duality equation, Proposition 3.3). Here, the spinor kernel $\psi\left(z_{1}, z_{2}\right)$ does have, order by order in $1 / N$, poles at ramification points. So, we do not have a simple expression for $\psi\left(z_{1}, z\right) \psi\left(z, z_{2}\right)$. However, Theorem 8.2 shows all contributions from the ramification points cancel in the sum over sheets.

\subsection{Christoffel-Darboux relations}

The matrix $\Psi\left(x_{1}, x_{2}\right)$ is invertible, since it is defined as a series for which the leading term coincides with $\Psi_{\mathrm{cl}}\left(x_{1}, x_{2}\right)$ which is invertible (see Lemma 3.2). We also have a duality relation to express the inverse

Corollary 8.1. If Conjecture 7.1 holds,

$$
\Psi^{-1}\left(x_{1}, x_{2}\right)=-\frac{\left(x_{1}-x_{2}\right)^{2}}{\mathrm{~d} x_{1} \mathrm{~d} x_{2}} \Psi\left(x_{2}, x_{1}\right) .
$$

Theorem 8.3. If Conjecture 7.1 holds, the matrices $\Psi(x)$ and $\Phi(x)$ are invertible, and we have the Christoffel-Darboux relation

$$
\psi\left(z_{1}, z_{2}\right)=\frac{\sum_{I, J} \psi_{I}\left(z_{1}\right) A_{I, J} \phi_{J}\left(z_{2}\right)}{X\left(z_{1}\right)-X\left(z_{2}\right)},
$$

where the matrix $A$ is invertible, independent of $x$ and given by

$$
A^{-1}=\frac{1}{\mathrm{~d} x} \Phi(x) \Psi^{t}(x) .
$$

The proofs can be copied from the semiclassical case (Section 3.4), because they are based only on the duality equation. 


\subsection{Differential systems}

$\Psi\left(x_{1}, x_{2}\right)$ is the solution of a system of differential equations with respect to the positions of the poles $X(p)(x=\infty$ is a fixed pole $)$ and the times $t_{p, j}$.

Theorem 8.4. If Conjecture 7.1 holds, then for any deformation parameter $\lambda=t_{p, j}$ or $\lambda=$ $X(p) \neq \infty$, there exists a $d \times d$ matrix $M_{\lambda}\left(x_{1}, x_{2}\right)$, such that

(i) $\left(\partial_{\lambda}-M_{\lambda}\right) \Psi\left(x_{1}, x_{2}\right)=0$;

(ii) $M_{\lambda}$ is a rational function of $x_{2}$;

(iii) $M_{\lambda}$ has no pole at branchpoints.

These deformations are compatible (since $\Psi$ is invertible) and isomonodromic.

Implicitly, for $j=0$, only deformations $\partial_{\lambda}=\partial_{t_{p, 0}}-\partial_{t_{p^{\prime}, 0}}$ are considered.

Proof. By definition

$$
M_{\lambda}=\partial_{\lambda} \Psi\left(x_{1}, x_{2}\right) \Psi^{-1}\left(x_{1}, x_{2}\right)=-\frac{\left(x_{1}-x_{2}\right)^{2}}{\mathrm{~d} x_{1} \mathrm{~d} x_{2}} \partial_{\lambda} \Psi\left(x_{1}, x_{2}\right) \Psi\left(x_{2}, x_{1}\right) .
$$

When $\lambda$ is a time $t_{p, k}(k \geq 1)$, we have by self-replication

$$
\partial_{t_{p, k}} \psi\left(z_{1}, z_{2}\right)=-\int_{\omega_{p, k}^{*}} \psi\left(z_{1}, \cdot\right) \psi\left(\cdot, z_{2}\right) .
$$

We compute

$$
\begin{aligned}
{\left[M_{t_{p, k}}\right]_{i j} } & =\frac{\left(x_{1}-x_{2}\right)^{2}}{\mathrm{~d} x_{1} \mathrm{~d} x_{2}} \sum_{m} \int_{\omega_{p, k}^{*}} \psi\left(z_{1}^{i}, z\right) \psi\left(z, z_{2}^{m}\right) \psi\left(z_{2}^{m}, z_{1}^{j}\right) \\
& =\frac{1}{\mathrm{~d} x_{1}} \int_{\omega_{p, k}^{*}} \frac{\left(X(\cdot)-x_{1}\right)\left(x_{2}-x_{1}\right)}{\left(X(\cdot)-x_{2}\right)} \psi\left(z_{1}^{i}, \cdot\right) \psi\left(\cdot, z_{1}^{j}\right) .
\end{aligned}
$$

It is clear that $M_{t_{p, k}}\left(x_{1}, x_{2}\right)$ is a rational function of $x_{2}$ without poles at branchpoints. If we assume $X(p) \neq \infty$, it has a pole only at $x_{2}=X(p)$, and this pole is of order $k+1$. If we assume $X(p)=\infty$, it has a pole only at $x_{2}=\infty$, and this pole is of order $1+\left\lfloor k / d_{p}\right\rfloor$ (we recall that $d_{p}$ is the multiplicity of order of the pole $p$ of $X$ ). It is straightforward to adapt this proof for $k=0$, namely $\partial_{\lambda}=\partial_{t_{p, 0}}-\partial_{t_{p^{\prime}, 0}}$.

Now, we turn to $\lambda \stackrel{p^{\prime}, 0}{=} X(p) \neq \infty$. We have assumed that $\mathrm{d} X(p) \neq 0$, so the preimages $p^{1}, \ldots, p^{d}$ of $X(p)$ are distinct, and $(X(z)-X(p))$ is a local coordinate near each $p^{m}$. The Laurent expansion of $Y \mathrm{~d} X(z)$ when $z \rightarrow p^{m}$ is

$$
Y \mathrm{~d} X(z)=\sum_{k \geq 0} t_{p, k} \frac{\mathrm{d} X(z)}{(X(z)-X(p))^{k+1}}+O(1),
$$

where only a finite number of $t_{p, k^{\prime}}=0$ are non zero. If we perform $X(p) \rightarrow X(p)+\lambda$ while the times are fixed, we change $Y \mathrm{~d} X$ to $(Y \mathrm{~d} X)_{\lambda}$, with Laurent expansion at $p^{m}$

$$
(Y \mathrm{~d} X)_{\lambda}=Y \mathrm{~d} X+\lambda\left(\sum_{k \geq 1} \frac{k t_{p, k-1}}{(X(z)-X(p))^{k+1}}\right)+O(1) .
$$

So, we can identify

$$
\frac{\partial}{\partial X(p)} \longrightarrow \sum_{k \geq 1} k t_{p, k-1} \frac{\partial}{\partial t_{p, k}}
$$


which is a finite sum. Hence

$$
\left[M_{X(p)}\right]_{i j}=-\frac{1}{\mathrm{~d} x_{1}} \sum_{m} \operatorname{Res}_{z \rightarrow z^{m}(X(p))} \frac{\mathrm{d} Y(z)}{\mathrm{d} X(z)} \frac{\left(X(z)-x_{1}\right)\left(x_{2}-x_{1}\right)}{\left(X(z)-x_{2}\right)} \psi\left(z_{1}^{i}, z\right) \psi\left(z, z_{1}^{j}\right) .
$$

This is a rational fraction of $x_{2}$, with a pole at $x_{2}=X(p)$ of order

$$
1+\left\lfloor\left(\max _{m} \operatorname{ord}_{z^{m}(p)} Y \mathrm{~d} X\right) / d_{p}\right\rfloor .
$$

One could also define a matrix $L$ with the reconstruction formula presented in Section 3.5. But now, the function $Y$ depends on the spectral curve, the evolution of $L$ under the flows generated by the times is not isospectral. However, since the position and the order of the poles is fixed, the deformation parameters $t_{p, j}$ preserve the monodromies of $\Psi$. Such a reconstruction has also been performed at leading order by Bertola and Gekhtman [13], and our expressions up to $o(1)$ match their results.

\section{Dictionary for matrix models}

We recall here the correspondence between the definitions in integrability (and in this article), and the observables defined in matrix models. Let $M$ be a random square matrix of size $N \times N$, diagonalizable by a unitary conjugation, with eigenvalues restricted to some contour $\Gamma$ in the complex plane. The probability measure $\mathrm{d} \mu(M)$ is a data of the matrix model. Its normalization defines the partition function $Z_{N}=\int \mathrm{d} \mu(M)$. For a function $f$, we note $\langle f(M)\rangle$ the expectation value of the random variable $f(M)$

$$
\langle f(M)\rangle=\frac{\int \mathrm{d} \mu(M) f(M)}{\int \mathrm{d} \mu(M)} .
$$

\subsection{Examples of integrable matrix models}

Important examples of integrable matrix models, described in details in [69], include:

The one hermitian matrix model. $M \in H_{N}$ is a $N \times N$ hermitian matrix, with eigenvalues on the real axis

$$
\mathrm{d} \mu(M)=\mathrm{e}^{-N \operatorname{Tr} V(M)} \mathrm{d} M, \quad \mathrm{~d} M=\prod_{i=1}^{N} \mathrm{~d} M_{i, i} \prod_{1 \leq i<j \leq N} \mathrm{~d} \operatorname{Re} M_{i, j} \mathrm{~d} \operatorname{Im} M_{i, j},
$$

and $V(M)$ is a "semiclassical potential" (see [7]), i.e. $V^{\prime}(x)$ is a rational function of its variable $x$, chosen such that $\int_{\mathbb{R}} \mathrm{e}^{-V(x)} \mathrm{d} x$ is absolutely convergent.

The one normal matrix model, with eigenvalues on a contour $\boldsymbol{\Gamma}$. We define

$$
H_{N}(\Gamma)=\left\{U \operatorname{diag}\left(x_{1}, \ldots, x_{N}\right) U^{\dagger}, U \in U(N) \text { and }\left(x_{1}, \ldots, x_{N}\right) \in \Gamma^{N}\right\}
$$

equipped with a "measure" (not necessarily real or normalized) ${ }^{3}$

$$
\mathrm{d} M=\mathrm{d} U \prod_{i<j}\left(x_{i}-x_{j}\right)^{2} \prod_{i=1}^{N} \mathrm{~d} x_{i}
$$

\footnotetext{
${ }^{3}$ For a given $M \in H_{N}(\Gamma)$, there are many choices of $U$ and $x_{i}$ 's (in fact $U$ can be multiplied by any element of $U(1)^{N}$ and the $x_{i}$ 's can be permuted, and $\mathrm{d} U$ is in fact the measure on the quotient $U(N) / \mathfrak{S}_{N} \times U(1)^{N}$ by the Haar measure on $U(N)$.
} 
where $\mathrm{d} U$ is the Haar measure on the unitary group $U(N)$ and $\mathrm{d} x_{i}$ is the curvilinear ${ }^{4}$ measure along $\Gamma$. This measure is always invariant under unitary transformations. We then define the measure $\mathrm{d} \mu(M)$ on $H_{N}(\Gamma)$

$$
\mathrm{d} \mu(M)=\mathrm{e}^{-N \operatorname{Tr} V(M)} \mathrm{d} M
$$

where $V^{\prime}(x) \in \mathbb{C}(x)$, i.e. $V^{\prime}(x)$ is a rational function of its variable $x$, chosen such that $\int_{\Gamma} \mathrm{e}^{-V(x)} \mathrm{d} x$ is absolutely convergent. This means that for a given potential $V(x), \Gamma$ must go to $\infty$ or to the poles of $V^{\prime}$, only in sectors where $\operatorname{Re} V(x) \rightarrow+\infty$. When $\Gamma=\mathbb{R}$, this definition is the same as the hermitian matrix model $H_{N}(\mathbb{R})=H_{N}$, with the usual Lebesgue measure on $H_{N}$. When $\Gamma$ is the unit circle $\mathbb{S}_{1}$, this definition is that of the unitary matrix model $H_{N}\left(\mathbb{S}_{1}\right)=U(N)$.

The two normal matrices model. Given two paths $\Gamma_{1}, \Gamma_{2}$, we define a measure on $H_{N}\left(\Gamma_{1}\right) \times H_{N}\left(\Gamma_{2}\right)$

$$
\mathrm{d} \mu_{2}\left(M_{1}, M_{2}\right)=\mathrm{e}^{-N \operatorname{Tr}\left[V_{1}\left(M_{1}\right)+V_{2}\left(M_{2}\right)-M_{1} M_{2}\right]} \mathrm{d} M_{1} \mathrm{~d} M_{2},
$$

where $V_{1}$ and $V_{2}$ are semiclassical potentials ( $V_{1}^{\prime}$ and $V_{2}^{\prime}$ are rational functions), chosen such that integrals on $\Gamma_{1} \times \Gamma_{2}$ are absolutely convergent. Upon integration on $M_{2}$, this measure induces a measure $\mathrm{d} \mu(M)$ on $M_{1}$ that we rename $M$

$$
\mathrm{d} \mu\left(M_{1}\right)=\int_{M_{2} \in H_{N}\left(\Gamma_{2}\right)} \mathrm{d} \mu_{2}\left(M, M_{2}\right) .
$$

The chain of matrices. This is the natural generalization of the case of two matrices. Consider $k$ paths $\Gamma_{1}, \ldots, \Gamma_{k}$, and $k$ semiclassical potentials $V_{1}, \ldots, V_{k}$, and define the measure on $H_{N}\left(\Gamma_{1}\right) \times \cdots \times H_{N}\left(\Gamma_{k}\right)$ as

$$
\mathrm{d} \mu_{k}\left(M_{1}, \ldots, M_{k}\right)=\mathrm{e}^{-N \operatorname{Tr}\left[\sum_{i=1}^{k} V_{i}\left(M_{i}\right)-\sum_{i=1}^{k-1} M_{i} M_{i+1}\right]} \mathrm{d} M_{1} \cdots \mathrm{d} M_{k}
$$

For any $i \in\{1, \ldots, k\}$, we integrate on $M_{j}$ 's with $j \neq i$, and renaming $M=M_{i}$, this measure induces a measure $\mathrm{d} \mu(M)$ :

$$
\mathrm{d} \mu\left(M_{i}\right)=\int_{M_{j} \in H_{N}\left(\Gamma_{j}\right), j \neq i} \mathrm{~d} \mu_{k}\left(M_{1}, \ldots, M_{k}\right) .
$$

\subsection{Correspondences}

\subsubsection{Partition function}

All the listed matrix models above have the property to be integrable, in a sense explained below. There is a huge literature on the subject, let us mention among others the early works in physics $[57,61]$, and in mathematics $[1,46,78]$. For the one and two matrices models, it is established that the partition function $Z_{N}=\int \mathrm{d} \mu(M)$ is an isomonodromic Tau function [10, 11, 14], but such a result is not known at present for the chain of matrices. The so-called double scaling limit of matrix models has been also intensively from the point of view of isomonodromic deformations [28, 43, 50, 70].

\footnotetext{
${ }^{4}$ If $\Gamma \subset \mathbb{C}$ is a path in the complex plane parametrized by a $C^{1}$ function $\gamma: \mathbb{R} \rightarrow \Gamma \subset \mathbb{C}$, i.e. $\Gamma=\{\gamma(s), s \in \mathbb{R}\}$, at $x=\gamma(s)$ we define the curvilinear measure $\mathrm{d} x=\gamma^{\prime}(s) \mathrm{d} s$ where $\mathrm{d} s$ is the Lebesgue measure on $\mathbb{R}$.
} 


\subsubsection{Correlators, spectral curves, loop equations}

For all the matrix models above, the correlators are

$$
\mathcal{W}_{n}\left(x_{1}, \ldots, x_{n}\right)=N^{-n}\left\langle\prod_{i=1}^{n} \operatorname{Tr} \frac{1}{x_{i}-M}\right\rangle_{C}, \quad \overline{\mathcal{W}}_{n}\left(x_{1}, \ldots, x_{n}\right)=N^{-n}\left\langle\prod_{i=1}^{n} \operatorname{Tr} \frac{1}{x_{i}-M}\right\rangle,
$$

where $C$ means 'cumulant'. $\mathcal{W}_{n}$ is often called $n$-point function, or connected $n$-point function, and $\overline{\mathcal{W}}_{n}$ is called disconnected $n$-point function.

The one point function $\mathcal{W}_{1}(x)$ (also called resolvent) plays an important role. In all the matrix models above, under reasonable assumptions, it has a large $N$ limit denoted

$$
\frac{1}{N} \mathcal{W}_{1}(x) \underset{N \rightarrow \infty}{\sim} \mathcal{W}_{1}^{(0)}(x)=\mathcal{Y}(x),
$$

which is furthermore an algebraic function of $x$, i.e. there exists a polynomial $\mathcal{E}(x, y)$ such that $\mathcal{E}(x, \mathcal{Y}(x))=0$. Hence, there exists a spectral curve $\mathcal{S}_{\mathrm{MM}}=(\mathcal{C}, X, Y)$, such that

$$
\forall z \in \mathcal{C}, \quad \mathcal{Y}(X(z))=Y(z) .
$$

If we define

$$
W_{n}\left(z_{1}, \ldots, z_{n}\right)=N^{n} \mathcal{W}_{n}\left(X\left(z_{1}\right), \ldots, X\left(z_{n}\right)\right) \bigotimes_{i=1}^{n} \mathrm{~d} X\left(z_{i}\right)+\delta_{n, 2} \frac{\mathrm{d} X\left(z_{1}\right) \otimes \mathrm{d} X\left(z_{2}\right)}{\left(X\left(z_{1}\right)-X\left(z_{2}\right)\right)^{2}}
$$

it is a classical result of random matrix theory (for instance it can be proved by integration by parts in the matrix integral) that, for all matrix models listed above, $W_{n}$ satisfy the loop equations of Theorem 6.1 .

\subsubsection{Large $N$ asymptotic expansion}

It is conjectured that the large $N$ asymptotic expansion of the partition function $Z_{N}$ matches with that of $\mathcal{T}\left[\mathcal{S}_{\mathrm{MM}}\right]$ function for the spectral curve $\mathcal{S}$ introduced in Definition 5.4, i.e. has an asymptotic expansion

$$
\ln Z_{N}=N^{2} F_{0}+F_{1}+\ln \Theta+\frac{1}{N}\left(F_{1}^{\prime} \frac{\Theta^{\prime}}{\Theta}+F_{0}^{\prime \prime \prime} \frac{\Theta^{\prime \prime \prime}}{\Theta}\right)+o(1 / N) .
$$

The characteristics $[\mu, \nu]$ of the Theta function $\Theta$ is determined by the choice of the integration contour $\Gamma$. This conjecture was derived heuristically in $[17,35]$.

When the semiclassical spectral curve is of genus 0 , there is no Theta function and the expansion involves only powers of $1 / N$ (in fact, powers of $1 / N^{2}$ ). This happens for the socalled "one-cut regime", and for the one matrix model, the existence of such an expansion has been proved for the one matrix model for real-valued, analytic potential [3], and then the coefficients are necessarily given by the symplectic invariants of the semiclassical spectral curve [36]. Beyond the one-cut regime, the Riemann-Hilbert steepest descent analysis [26, 50] has been applied to find explicitly the asymptotics up to $o(1)$ in the one hermitian matrix model with real-valued, polynomial potential $[16,24]$. It features in general a pseudo-periodic behavior with $N$, encoded in the Theta function, but the one-cut regime can also be retrieved with this Riemann-Hilbert method [34]. These results have later been extended to the one normal matrix model with complex-valued polynomial potential [15]. This method can be used in principle to find recursively the subleading orders, although it does not allow to write the answer a priori explicitly to all orders. Definition 5.4 is expected to give the correct answer to all orders. 


\subsubsection{Baker-Akhiezer spinor kernel}

The spinor kernel is related to the expectation value of ratios of characteristic polynomials

$$
\psi\left(z_{1}, z_{2}\right)=\left\langle\frac{\operatorname{det}\left(X\left(z_{1}\right)-M\right)}{\operatorname{det}\left(X\left(z_{2}\right)-M\right)}\right\rangle \frac{\sqrt{\mathrm{d} X\left(z_{1}\right) \mathrm{d} X\left(z_{2}\right)}}{X\left(z_{1}\right)-X\left(z_{2}\right)} .
$$

The Baker-Akhiezer spinor kernel $\psi_{\mathrm{cl}}\left(z_{1}, z_{2}\right)$ is the large $N$ limit of $\psi\left(z_{1}, z_{2}\right)$. When sending $X\left(z_{2}\right) \rightarrow \infty$, one gets, after proper renormalization

$$
\psi\left(z_{1}\right)=" \psi\left(z_{1}, \infty\right) "=\left\langle\operatorname{det}\left(X\left(z_{1}\right)-M\right)\right\rangle,
$$

which is clearly a polynomial in $X\left(z_{1}\right)$ of degree $N$. It is a classical result of random matrix theory [69] that this expectation value of the characteristic polynomial of $M$ is the orthogonal polynomial of degree $N$, for the orthogonality given by the measure $\mathrm{d} \mu$ for matrices of size 1 . The dual function for matrix models is

$$
\phi\left(z_{1}\right)=" \psi\left(\infty, z_{1}\right) "=\left\langle\frac{1}{\operatorname{det}\left(X\left(z_{1}\right)-M\right)}\right\rangle .
$$

It can be derived from orthogonality relations of the orthogonal polynomials that those spinor kernels satisfy Hirota equations, also called "determinantal formula", for instance,

$$
\begin{gathered}
\frac{\left(x_{1}-x_{2}\right)\left(x_{3}-x_{4}\right)}{\left(x_{1}-x_{3}\right)\left(x_{1}-x_{4}\right)\left(x_{2}-x_{3}\right)\left(x_{3}-x_{4}\right)}\left\langle\frac{\operatorname{det}\left(x_{1}-M\right) \operatorname{det}\left(x_{2}-M\right)}{\operatorname{det}\left(x_{3}-M\right) \operatorname{det}\left(x_{4}-M\right)}\right\rangle \\
=\frac{1}{\left(x_{1}-x_{3}\right)\left(x_{2}-x_{4}\right)}\left\langle\frac{\operatorname{det}\left(x_{1}-M\right)}{\operatorname{det}\left(x_{3}-M\right)}\right\rangle\left\langle\frac{\operatorname{det}\left(x_{2}-M\right)}{\operatorname{det}\left(x_{4}-M\right)}\right\rangle \\
-\frac{1}{\left(x_{1}-x_{4}\right)\left(x_{2}-x_{3}\right)}\left\langle\frac{\operatorname{det}\left(x_{1}-M\right)}{\operatorname{det}\left(x_{4}-M\right)}\right\rangle\left\langle\frac{\operatorname{det}\left(x_{2}-M\right)}{\operatorname{det}\left(x_{3}-M\right)}\right\rangle .
\end{gathered}
$$

In that case, $N$ is an integer (the size of the matrices), and Hirota equation is an equality between sequences indexed by $N$, not only of formal asymptotic series. This relations have been proved in [44] for the one matrix model, and in [5, 2] for the two matrices model, from which the case of chain of matrices can be deduced. It shows that, modulo the heuristic derivation of asymptotics of matrix integrals, Hirota equations (as stated as in Conjecture 7.4) hold for the semiclassical spectral curves of matrix models (however, not all algebraic curves can be reached in this way).

\subsubsection{Differential systems}

The orthogonal polynomials, as well as their duals, in all cases above, do satisfy ODE's of order $d$ ( $d$ depends on the degrees of potentials). As we have seen in Section 8 , the proof is a consequence of Hirota equations. For instance for the one matrix model, this is a second order ODE $(d=2)$. For the two matrices model with potentials $V_{1}$ and $V_{2}$, we always have $d=1+\operatorname{deg} V_{2}^{\prime}$ (in case $V_{2}^{\prime}$ is a rational function, $\operatorname{deg} V_{2}^{\prime}$ is the sum of degrees of all poles). In all cases we have a $d$-dimensional vector

$$
\vec{\Psi}(z)=\left(\begin{array}{c}
\psi\left(z_{1}\right) \\
\vdots
\end{array}\right),
$$

where the first entry is $\psi\left(z_{1}\right)$. The other entries are obtained from $\psi\left(z_{1}\right)$ with a procedure described in [8] and very similar to Section 3.3. This vector satisfies an ODE

$$
\frac{\mathrm{d}}{\mathrm{d} X(z)} \vec{\Psi}(z)=\mathcal{D}(X(z)) \vec{\Psi}(z)
$$


where $\mathcal{D}(x)$ is a $d \times d$ matrix, whose entries are rational functions of $x$, that depend implicitly on $N$, on the coefficients of the potentials, and on the choice of integration contour $\Gamma$. The locus of eigenvalues of $\mathcal{D}(x)$, i.e. the polynomial equation

$$
\mathcal{E}_{N}(x, y)=\operatorname{det}(y-\mathcal{D}(x))=0
$$

defines a spectral curve for any finite $N$. The semiclassical spectral curve (i.e. its large $N$ limit) coincides with the spectral curve $\mathcal{S}_{\mathrm{MM}}$

$$
\mathcal{E}(x, y)=\lim _{N \rightarrow \infty} \mathcal{E}_{N}(x, y)
$$

There are also $d \times d$ differential systems for derivatives with respect to all coefficients of the potentials, and there is also a linear recursion relation on $N \rightarrow N+1$ (see [23] for the one matrix model). All these systems are compatible as shown in [9] in full generality for the chain of matrices.

\subsubsection{Symplectic invariance}

Notice that, in the two matrices model

$$
Z=\int_{H_{N}(\Gamma) \times H_{N}(\tilde{\Gamma})} \mathrm{d} \mu\left(M_{1}, M_{2}\right),
$$

we have defined our semiclassical spectral curve $\mathcal{S}=(\mathcal{C}, X, Y)$ from the large $N$ limit of the resolvent $\mathcal{W}_{1}(x)=\left\langle\operatorname{tr} \frac{1}{x-M_{1}}\right\rangle$ associated to the matrix $M_{1}$. Since $M_{1}$ and $M_{2}$ play a symmetric role, it is clear that we would have obtained the same partition function, starting from the semiclassical spectral curve $\tilde{\mathcal{S}}=(\tilde{\mathcal{C}}, \tilde{X}, \tilde{Y})$ associated to the resolvent of matrix $M_{2}$, and thus we must have

$$
\mathcal{T}[\mathcal{S}]=\mathcal{T}[\tilde{\mathcal{S}}] .
$$

One can easily find that the two spectral curves $\mathcal{S}_{\mathrm{MM}}=(\mathcal{C}, X, Y)$ and $\tilde{\mathcal{S}}_{\mathrm{MM}}=(\tilde{\mathcal{C}}, \tilde{X}, \tilde{Y})$ are related by $\mathcal{C}=\tilde{\mathcal{C}}$ and $\tilde{X}=Y, \tilde{Y}=X$, in other words they arse symplectically equivalent. Hence, the fact that $\mathcal{T}\left[\mathcal{S}_{\mathrm{MM}}\right]=\mathcal{T}\left[\tilde{\mathcal{S}}_{\mathrm{MM}}\right]$ can be seen at all order in the large $N$ expansion as a consequence of the symplectic invariance of the $F_{g}$ 's. In fact, this is a manifestation at large $N$ of an exact result for finite $N$. The orthogonal polynomials $\vec{\Psi}(x)$ associated to matrix $M_{1}$ satisfy an ODE of some order $d$ (see equation (9.1))

$$
\frac{\mathrm{d}}{\mathrm{d} X(z)} \vec{\Psi}(z)=\mathcal{D}(X(z)) \vec{\Psi}(z)
$$

whereas the orthogonal polynomials $\Psi \overrightarrow{\tilde{(}} y)$ associated to matrix $M_{2}$ satisfy another ODE of some order $\tilde{d}$ (in general $\tilde{d} \neq d$ )

$$
\frac{\mathrm{d}}{\mathrm{d} Y(z)} \overrightarrow{\tilde{\Psi}}(z)=\tilde{\mathcal{D}}(Y(z)) \overrightarrow{\tilde{\Psi}}(z) .
$$

It was discovered in [12] that

$$
\operatorname{det}\left(y \mathbf{1}_{d \times d}-\mathcal{D}(x)\right)=\operatorname{det}\left(x \mathbf{1}_{\tilde{d} \times \tilde{d}}-\tilde{\mathcal{D}}(y)\right) .
$$

This also implies that the semiclassical spectral curve defined from $M_{1}$ or from $M_{2}$ are related as explained above. 


\section{Conclusion}

For integrable systems with a small dispersive parameter $1 / N$, using the theory of symplectic invariants [38], we have introduced a formal object $\mathcal{T}$, which is conjectured to be a Tau function in the sense that it satisfies Hirota equations. It is challenging to find a full proof of Conjecture 7.4, and that would certainly prove that a certain quantity constructed out of $\mathcal{T}$ (like $u(x, t)=$ $2(\ln \mathcal{T})_{x x}$ for $\left.\mathrm{KdV}\right)$ provides the all-order asymptotics of solutions of nonlinear integrable PDE's in the small dispersion limit.

One can wonder how to generalize the construction. First, to algebraic curves in $\mathbb{C}^{*} \times \mathbb{C}^{*}$, having in view the mirror curves appearing in Gromov-Witten theory, which all are of the form $\operatorname{Pol}\left(e^{x}, e^{y}\right)=0$. In a recent work, we have described an application of the expressions for $\mathcal{T}$ and $\psi$ for such curves to the computation of perturbative knot invariants [18]. This explicit example suggests that the construction remains meaningful in this context. Second, it should be possible to extend our construction to the bundles appearing in generalized matrix models like the $O(n)$ model, which might be related to isomonodromic deformations on Riemann surfaces of positive genus and to Hitchin systems [48]. And, even further to $\mathcal{D}$-modules, for which an adapted topological recursion (the so-called $\beta$-deformation of the topological recursion) is being developed with similar properties [21, 22].

Eventually, it remains to compare this construction with other approaches (Frobenius manifolds [29] and construction of integrable hierarchies of topological type [33], Poisson bracket structures, Segal-Wilson formalism in the Grassmannian [76], etc.), study its consequences and better understand the underlying geometry.

\section{A Proof of Conjecture 7.1 up to $o(1 / N)$}

Proposition A.1. $\psi\left(z_{1}, z_{2}\right)$ is self-replicating at least up to o $(1 / N)$,

$$
\frac{1}{N} \delta_{z} \psi\left(z_{1}, z_{2}\right)+\psi\left(z_{1}, z\right) \psi\left(z, z_{2}\right)=o(1 / N)
$$

Proof. Let us start from $\psi_{12}=\psi\left(z_{1}, z_{2}\right)$ written as

$$
\psi_{12}=\frac{\mathrm{e}^{N \int_{2}^{1} Y \mathrm{~d} X}}{E_{12}} \frac{\Theta_{12}}{\Theta}\left\{1+\frac{1}{N} \hat{\psi}_{12}+o(1 / N)\right\},
$$

with

$$
\begin{aligned}
\hat{\psi}_{12}= & \int_{2}^{1} \omega_{1}^{(1)}+\frac{1}{6} \int_{2}^{1} \int_{2}^{1} \int_{2}^{1} \omega_{3}^{(0)}+\frac{1}{2} \frac{\Theta_{12}^{\prime \prime}}{\Theta_{12}} \oint \oint \int_{2}^{1} \omega_{3}^{(0)}+\frac{1}{2} \frac{\Theta_{12}^{\prime}}{\Theta_{12}} \oint \int_{2}^{1} \int_{2}^{1} \omega_{3}^{(0)} \\
& +\left(\frac{\Theta_{12}^{\prime}}{\Theta_{12}}-\frac{\Theta^{\prime}}{\Theta}\right) F_{1}^{\prime}+\frac{1}{6}\left(\frac{\Theta_{12}^{\prime \prime \prime}}{\Theta_{12}}-\frac{\Theta^{\prime \prime \prime}}{\Theta}\right) F_{0}^{\prime \prime \prime}
\end{aligned}
$$

where, to shorten notations, 1 means $z_{1}, 2$ means $z_{2}, \oint$ means the contour integral around $\mathcal{B}$ cycles (indices are understood in tensor notations, i.e. contracted with the indices of derivatives of $\Theta$ ), and $\Theta_{12}$ means

$$
\Theta_{12}=\Theta\left(\mathbf{w}_{0}+2 \mathrm{i} \pi\left(\mathbf{u}\left(z_{1}\right)-\mathbf{u}\left(z_{2}\right)\right)\right), \quad \Theta=\Theta\left(\mathbf{w}_{0}\right) .
$$

We remind that (equation (5.1))

$$
\omega_{3}^{(0)}\left(z_{0}, z_{1}, z_{2}\right)=\sum_{i} \operatorname{Res}_{z \rightarrow a_{i}} \frac{B\left(z_{0}, z\right) B\left(z_{1}, z\right) B\left(z_{2}, z\right)}{\mathrm{d} X(z) \mathrm{d} Y(z)},
$$


and by special geometry

$$
F_{0}^{\prime \prime \prime}=\oint_{\mathcal{B}} \oint_{\mathcal{B}} \oint_{\mathcal{B}} \omega_{3}^{(0)}=\sum_{i} \operatorname{Res}_{z \rightarrow a_{i}} \frac{(\mathrm{d} \mathbf{v}(z))^{3}}{\mathrm{~d} X(z) \mathrm{d} Y(z)},
$$

where we have set

$$
\mathrm{d} \mathbf{v}(z)=2 \mathrm{i} \pi \mathrm{d} \mathbf{u}(z)=\oint_{\mathcal{B}} B(z, \cdot) .
$$

The expression for $\hat{\psi}_{12}$ is thus

$$
\begin{aligned}
\hat{\psi}_{12}= & \int_{2}^{1} \omega_{1}^{(1)}+\frac{1}{6} \int_{2}^{1} \int_{2}^{1} \int_{2}^{1} \omega_{3}^{(0)}+\frac{1}{2} \frac{\Theta_{12}^{\prime \prime}}{\Theta_{12}} \oint \oint \int_{2}^{1} \omega_{3}^{(0)}+\frac{1}{2}\left(\frac{\Theta_{12}^{\prime}}{\Theta_{12}}\right) \oint \int_{2}^{1} \int_{2}^{1} \omega_{3}^{(0)} \\
& +\left(\frac{\Theta_{12}^{\prime}}{\Theta_{12}}-\frac{\Theta^{\prime}}{\Theta}\right) F_{1}^{\prime}+\frac{1}{6}\left(\frac{\Theta_{12}^{\prime \prime \prime}}{\Theta_{12}}-\frac{\Theta^{\prime \prime \prime}}{\Theta}\right) F_{0}^{\prime \prime \prime} \\
= & \int_{2}^{1} \omega_{1}^{(1)}+\left(\frac{\Theta_{12}^{\prime}}{\Theta_{12}}-\frac{\Theta^{\prime}}{\Theta}\right) F_{1}^{\prime}+\sum_{i} \operatorname{Res}_{z \rightarrow a_{i}} \frac{1}{\mathrm{~d} X(z) \mathrm{d} Y(z)}\left\{\frac{1}{6}\left(\mathrm{~d} S_{12}(z)\right)^{3}\right. \\
& +\frac{1}{2}\left(\frac{\Theta_{12}^{\prime}}{\Theta_{12}}\right) \mathrm{d} \mathbf{v}(z)\left(\mathrm{d} S_{12}(z)\right)^{2} \\
& \left.+\frac{1}{2}\left(\frac{\Theta_{12}^{\prime \prime}}{\Theta_{12}}\right)(\mathrm{d} \mathbf{v}(z))^{2} \mathrm{~d} S_{12}(z)+\frac{1}{6}\left(\frac{\Theta_{12}^{\prime \prime \prime}}{\Theta_{12}}-\frac{\Theta^{\prime \prime \prime}}{\Theta}\right)(\mathrm{d} \mathbf{v}(z))^{3}\right\} .
\end{aligned}
$$

We need to apply the insertion operator $\frac{1}{N} \delta_{z}$ to $\hat{\psi}_{12}$, and obtain the result up to $o(1)$. Only the variation of $N F_{0}^{\prime}$ appearing in the Theta functions contributes to this order

$$
\begin{aligned}
\frac{1}{N} \delta_{z} \hat{\psi}_{12}= & {\left[\left(\frac{\Theta_{12}^{\prime}}{\Theta_{12}}-\frac{\Theta^{\prime}}{\Theta}\right)^{\prime} F_{1}^{\prime}+\sum_{i} \operatorname{Res}_{z^{\prime} \rightarrow a_{i}} \frac{1}{\mathrm{~d} X\left(z^{\prime}\right) \mathrm{d} Y\left(z^{\prime}\right)}\left\{\frac{1}{2}\left(\frac{\Theta_{12}^{\prime}}{\Theta_{12}}\right)^{\prime} \mathrm{d} \mathbf{v}\left(z^{\prime}\right)\left(\mathrm{d} S_{12}\left(z^{\prime}\right)\right)^{2}\right.\right.} \\
& \left.\left.+\frac{1}{2}\left(\frac{\Theta_{12}^{\prime \prime}}{\Theta_{12}}\right)^{\prime}\left(\mathrm{d} \mathbf{v}\left(z^{\prime}\right)\right)^{2} \mathrm{~d} S_{12}\left(z^{\prime}\right)+\frac{1}{6}\left(\frac{\Theta_{12}^{\prime \prime \prime}}{\Theta_{12}}-\frac{\Theta^{\prime \prime \prime}}{\Theta}\right)^{\prime}\left(\mathrm{d} \mathbf{v}\left(z^{\prime}\right)\right)^{3}\right\}\right] \mathrm{d} \mathbf{v}(z)+o(1) .
\end{aligned}
$$

This allows us to compute

$$
\begin{aligned}
\frac{1}{N} \delta_{z} \ln \psi_{12}= & \mathrm{d} S_{12}(z)+\left(\frac{\Theta_{12}^{\prime}}{\Theta_{12}}-\frac{\Theta^{\prime}}{\Theta}\right) \mathrm{d} \mathbf{v}(z)+\frac{1}{2 N} \int_{2}^{1} \int_{2}^{1} \omega_{3}^{(0)}(z, \cdot, \cdot) \\
& +\frac{1}{N}\left(\frac{\Theta_{12}^{\prime}}{\Theta_{12}}\right) \oint \int_{2}^{1} \omega_{3}^{(0)}(z, \cdot, \cdot)+\frac{1}{2 N}\left(\frac{\Theta_{12}^{\prime \prime}}{\Theta_{12}}-\frac{\Theta^{\prime \prime}}{\Theta}\right) \oint \oint \omega_{3}^{(0)}(z, \cdot, \cdot) \\
& +\frac{1}{N^{2}} \delta_{z} \hat{\psi}_{12}+o(1 / N) \\
= & \mathrm{d} S_{12}(z)+\mathrm{d} \mathbf{v}(z)\left(\frac{\Theta_{12}^{\prime}}{\Theta_{12}}-\frac{\Theta^{\prime}}{\Theta}\right)+\frac{1}{N} \sum_{i} \operatorname{Res}_{z^{\prime} \rightarrow a_{i}} \frac{B\left(z^{\prime}, z\right)}{\mathrm{d}\left(z^{\prime}\right) \mathrm{d} Y\left(z^{\prime}\right)}\left\{\frac{1}{2}\left(\mathrm{~d} S_{12}(z)\right)^{2}\right. \\
& \left.+\left(\frac{\Theta_{12}^{\prime}}{\Theta_{12}}\right) \mathrm{d} \mathbf{v}\left(z^{\prime}\right) \mathrm{d} S_{12}\left(z^{\prime}\right)+\frac{1}{2}\left(\frac{\Theta_{12}^{\prime \prime}}{\Theta_{12}}-\frac{\Theta^{\prime \prime}}{\Theta}\right)\left(\mathrm{d} \mathbf{v}\left(z^{\prime}\right)\right)^{2}\right\}+\frac{1}{N^{2}} \delta_{z} \hat{\psi}_{12}+o(1 / N) \\
= & \mathrm{d} S_{12}(z)+\left(\frac{\Theta_{12}^{\prime}}{\Theta_{12}}-\frac{\Theta^{\prime}}{\Theta}\right) \mathrm{d} \mathbf{v}(z)+\left(\frac{\Theta_{12}^{\prime}}{\Theta_{12}}-\frac{\Theta^{\prime}}{\Theta}\right)^{\prime} F_{1}^{\prime} \mathrm{d} \mathbf{v}(z) \\
& +\frac{1}{N} \sum_{i} \operatorname{Res}_{z^{\prime} \rightarrow a_{i}} \frac{1}{\mathrm{~d} X\left(z^{\prime}\right) \mathrm{d} Y\left(z^{\prime}\right)}\left\{\frac{1}{2}\left(\mathrm{~d} S_{12}\left(z^{\prime}\right)\right)^{2} B\left(z^{\prime}, z\right)\right.
\end{aligned}
$$




$$
\begin{aligned}
& +\left(\frac{\Theta_{12}^{\prime}}{\Theta_{12}}\right) \mathrm{d} \mathbf{v}\left(z^{\prime}\right) \mathrm{d} S_{12}\left(z^{\prime}\right) B\left(z^{\prime}, z\right)+\frac{1}{2}\left(\frac{\Theta_{12}^{\prime \prime}}{\Theta_{12}}-\frac{\Theta^{\prime \prime}}{\Theta}\right)\left(\mathrm{d} \mathbf{v}\left(z^{\prime}\right)\right)^{2} B\left(z^{\prime}, z\right) \\
& +\frac{1}{2}\left(\frac{\Theta_{12}^{\prime \prime}}{\Theta_{12}}\right)^{\prime}\left(\mathrm{d} \mathbf{v}\left(z^{\prime}\right)\right)^{2} \mathrm{~d} \mathbf{v}\left(z^{\prime}\right) \mathrm{d} S_{12}(z)+\frac{1}{2}\left(\frac{\Theta_{12}^{\prime}}{\Theta_{12}}\right)^{\prime} \mathrm{d} \mathbf{v}\left(z^{\prime}\right)\left(\mathrm{d} S_{12}\left(z^{\prime}\right)\right)^{2} \mathrm{~d} \mathbf{v}(z) \\
& \left.+\frac{1}{6}\left(\frac{\Theta_{12}^{\prime \prime \prime}}{\Theta_{12}}-\frac{\Theta^{\prime \prime \prime}}{\Theta}\right)^{\prime}\left(\mathrm{d} \mathbf{v}\left(z^{\prime}\right)\right)^{3} \mathrm{~d} \mathbf{v}(z)\right\}+o(1 / N)
\end{aligned}
$$

We may transform the first line using the refined duality equation established in Proposition 3.3, which is a consequence of the Fay identity satisfied by the Theta function of the spectral curve. It can be rephrased as

$$
\mathrm{d} S_{12}(z)+\left(\frac{\Theta_{12}^{\prime}}{\Theta_{12}}-\frac{\Theta^{\prime}}{\Theta}\right) \mathrm{d} \mathbf{v}(z)=-\frac{E_{12}}{E_{1 z} E_{z 2}} \frac{\Theta_{1 z} \Theta_{z 2}}{\Theta \Theta_{12}} .
$$

On the other hand we have

$$
\begin{aligned}
\frac{\psi_{1 z} \psi_{z 2}}{\psi_{12}}= & \frac{E_{12}}{E_{1 z} E_{z 2}} \frac{\Theta_{1 z} \Theta_{z 2}}{\Theta_{12}}\left(1+\frac{1}{N}\left(\hat{\psi}_{1 z}+\hat{\psi}_{z 2}-\hat{\psi}_{12}\right)+o(1 / N)\right) \\
= & \frac{E_{12}}{E_{1 z} E_{z 2}} \frac{\Theta_{1 z} \Theta_{z 2}}{\Theta_{12}}\left(1+\frac{1}{N}\left[\frac{\Theta_{1 z}^{\prime}}{\Theta_{1 z}}+\frac{\Theta_{z 2}^{\prime}}{\Theta_{z 2}}-\frac{\Theta_{12}^{\prime}}{\Theta_{12}}-\frac{\Theta^{\prime}}{\Theta}\right] F_{1}^{\prime}\right. \\
& +\frac{1}{N} \sum_{i} \operatorname{Res}_{z^{\prime} \rightarrow a_{i}} \frac{1}{\mathrm{~d} X\left(z^{\prime}\right) \mathrm{d} Y\left(z^{\prime}\right)}\left\{\frac { 1 } { 2 } \left(\frac{\Theta_{1 z}^{\prime}}{\Theta_{1 z}}\left(\mathrm{~d} S_{1 z}\left(z^{\prime}\right)\right)^{2}+\frac{\Theta_{z 2}^{\prime}}{\Theta_{z 2}}\left(\mathrm{~d} S_{z 2}\left(z^{\prime}\right)\right)^{2}\right.\right. \\
& \left.-\frac{\Theta_{12}^{\prime}}{\Theta_{12}}\left(\mathrm{~d} S_{12}\left(z^{\prime}\right)\right)^{2}\right) \mathrm{d} \mathbf{v}\left(z^{\prime}\right)+\frac{1}{2}\left(\frac{\Theta_{1 z}^{\prime \prime}}{\Theta_{1 z}} \mathrm{~d} S_{1 z}\left(z^{\prime}\right)+\frac{\Theta_{z 2}^{\prime \prime}}{\Theta_{z 2}} \mathrm{~d} S_{z 2}\left(z^{\prime}\right)\right. \\
& \left.\left.-\frac{\Theta_{12}^{\prime \prime}}{\Theta_{12}} \mathrm{~d} S_{12}\left(z^{\prime}\right)\right)\left(\mathrm{d} \mathbf{v}\left(z^{\prime}\right)\right)^{2}+\frac{1}{6}\left(\frac{\Theta_{1 z}^{\prime \prime \prime}}{\Theta_{1 z}}+\frac{\Theta_{z 2}^{\prime \prime \prime}}{\Theta_{z 2}}-\frac{\Theta_{12}^{\prime \prime \prime}}{\Theta_{12}}-\frac{\Theta^{\prime \prime \prime}}{\Theta}\right)\left(\mathrm{d} \mathbf{v}\left(z^{\prime}\right)\right)^{3}\right\} \\
& \left.-\frac{1}{2} \mathrm{~d} S_{1 z}\left(z^{\prime}\right) \mathrm{d} S_{z 2}\left(z^{\prime}\right) \mathrm{d} S_{12}\left(z^{\prime}\right)+o(1 / N)\right) .
\end{aligned}
$$

Let us now compute

$$
\begin{aligned}
\frac{1}{N} \delta_{z} \ln \psi_{12}+\frac{\psi_{1 z} \psi_{z 2}}{\psi_{12}}=\left\{\left(\frac{\Theta_{12}^{\prime}}{\Theta_{12}}-\frac{\Theta^{\prime}}{\Theta}\right)^{\prime} \mathrm{d} \mathbf{v}(z)\right. \\
\left.+\frac{E_{12}}{E_{1 z} E_{z 2}} \frac{\Theta_{1 z} \Theta_{z 2}}{\Theta \Theta_{12}}\left(\frac{\Theta_{1 z}^{\prime}}{\Theta_{1 z}}+\frac{\Theta_{z 2}^{\prime}}{\Theta_{z 2}}-\frac{\Theta_{12}^{\prime}}{\Theta_{12}}-\frac{\Theta^{\prime}}{\Theta}\right)\right\} F_{1}^{\prime} \\
+\sum_{i} \operatorname{Res}_{z^{\prime} \rightarrow a_{i}} \frac{1}{\mathrm{~d} X\left(z^{\prime}\right) \mathrm{d} Y\left(z^{\prime}\right)}\left\{\frac{1}{2}\left(\mathrm{~d} S_{12}\left(z^{\prime}\right)\right)^{2} B\left(z^{\prime}, z\right)+\left(\frac{\Theta_{12}^{\prime}}{\Theta_{12}}\right) \mathrm{d} \mathbf{v}\left(z^{\prime}\right) \mathrm{d} S_{12}\left(z^{\prime}\right) B\left(z^{\prime}, z\right)\right. \\
+\frac{1}{2}\left(\frac{\Theta_{12}^{\prime \prime}}{\Theta_{12}}-\frac{\Theta^{\prime \prime}}{\Theta}\right)\left(\mathrm{d} \mathbf{v}\left(z^{\prime}\right)\right)^{2} B\left(z^{\prime}, z\right)+\frac{1}{2}\left(\frac{\Theta_{12}^{\prime \prime}}{\Theta_{12}}\right)^{\prime}\left(\mathrm{d} \mathbf{v}\left(z^{\prime}\right)\right)^{2} \mathrm{~d} S_{12}\left(z^{\prime}\right) \mathrm{d} \mathbf{v}(z) \\
+\frac{1}{2}\left(\frac{\Theta_{12}^{\prime}}{\Theta_{12}}\right)^{\prime} \mathrm{d} \mathbf{v}\left(z^{\prime}\right)\left(\mathrm{d} S_{12}\left(z^{\prime}\right)\right)^{2} \mathrm{~d} \mathbf{v}(z)+\frac{1}{6}\left(\frac{\Theta_{12}^{\prime \prime \prime}}{\Theta_{12}}-\frac{\Theta^{\prime \prime \prime}}{\Theta}\right)^{\prime}\left(\mathrm{d} \mathbf{v}\left(z^{\prime}\right)\right)^{3} \mathrm{~d} \mathbf{v}(z) \\
+\frac{E_{12}}{E_{1 z} E_{z 2}} \frac{\Theta_{1 z} \Theta_{z 2}}{\Theta \Theta_{12}}\left[\frac{1}{2}\left(\frac{\Theta_{1 z}^{\prime}}{\Theta_{1 z}}\left(\mathrm{~d} S_{1 z}\left(z^{\prime}\right)\right)^{2}+\frac{\Theta_{z 2}^{\prime}}{\Theta_{z 2}}\left(\mathrm{~d} S_{z 2}\left(z^{\prime}\right)\right)^{2}-\frac{\Theta_{12}^{\prime}}{\Theta_{12}}\left(\mathrm{~d} S_{12}\left(z^{\prime}\right)\right)^{2}\right) \mathrm{d} \mathbf{v}\left(z^{\prime}\right)\right. \\
+\frac{1}{2}\left(\frac{\Theta_{1 z}^{\prime \prime}}{\Theta_{1 z}} \mathrm{~d} S_{1 z}\left(z^{\prime}\right)+\frac{\Theta_{z 2}^{\prime \prime}}{\Theta_{z 2}} \mathrm{~d} S_{z 2}\left(z^{\prime}\right)-\frac{\Theta_{12}^{\prime \prime}}{\Theta_{12}} \mathrm{~d} S_{12}\left(z^{\prime}\right)\right)\left(\mathrm{d} \mathbf{v}\left(z^{\prime}\right)\right)^{2}
\end{aligned}
$$




$$
\begin{aligned}
& \left.\left.+\frac{1}{6}\left(\frac{\Theta_{1 z}^{\prime \prime \prime}}{\Theta_{1 z}}+\frac{\Theta_{z 2}^{\prime \prime \prime}}{\Theta_{z 2}}-\frac{\Theta_{12}^{\prime \prime \prime}}{\Theta_{12}}-\frac{\Theta^{\prime \prime \prime}}{\Theta}\right)\left(\mathrm{d} \mathbf{v}\left(z^{\prime}\right)\right)^{3}-\frac{1}{2} \mathrm{~d} S_{1 z}\left(z^{\prime}\right) \mathrm{d} S_{z 2}\left(z^{\prime}\right) \mathrm{d} S_{12}\left(z^{\prime}\right)\right]\right\} \\
& +o(1 / N)
\end{aligned}
$$

The coefficient of $F_{1}^{\prime}$ vanishes, as we can see by computing the gradient of equation (A.1) to leading order in $N$ (recall that $N$ enters in the definition of our $\Theta$ through the point $\mathbf{w}_{0}=N F_{0}^{\prime}$ )

$$
\left(\frac{\Theta_{12}^{\prime}}{\Theta_{12}}-\frac{\Theta^{\prime}}{\Theta}\right)^{\prime} \mathrm{d} \mathbf{v}(z)-\frac{E_{12}}{E_{1 z} E_{z 2}} \frac{\Theta_{1 z} \Theta_{2 z}}{\Theta_{12}}\left(\frac{\Theta_{1 z}^{\prime}}{\Theta_{1 z}}+\frac{\Theta_{z 2}^{\prime}}{\Theta_{z 2}}-\frac{\Theta_{12}^{\prime}}{\Theta_{12}}-\frac{\Theta^{\prime}}{\Theta}\right)
$$

This identity can also be seen as a consequence of Fay identity.

Let us now study the residue term of equation (A.2), which we write

$$
\sum_{i} \operatorname{Res}_{z^{\prime} \rightarrow a_{i}} \frac{H_{12}\left(z^{\prime}, z\right)}{\mathrm{d} X\left(z^{\prime}\right) \mathrm{d} Y\left(z^{\prime}\right)}
$$

First, notice that by construction, $H_{12}\left(z^{\prime}, z\right)$ is a meromorphic 1 -form in the variable $z$, which means that it has trivial monodromy when $z$ goes around a non-trivial cycle. It may have simple poles at $z=z_{1}$ or $z=z_{2}$ coming from the ratio of prime forms, but the expression in $[\cdots]$ vanish when $z=z_{1}$ or $z_{2}$, so $H_{12}\left(z, z^{\prime}\right)$ is actually regular at $z=z_{1}$ or $z=z_{2}$. It may also have a singularity at $z=z^{\prime}$ coming from the term $[\cdots]$, which is at most a double pole. To leading order when $z \rightarrow z^{\prime}$, we find

$$
\begin{aligned}
H_{12}\left(z^{\prime}, z\right)= & \frac{1}{2} \mathrm{~d} S_{12}\left(z^{\prime}\right)^{2}+\left(\frac{\Theta_{12}^{\prime}}{\Theta_{12}}\right) \mathrm{d} \mathbf{v}\left(z^{\prime}\right) \mathrm{d} S_{12}\left(z^{\prime}\right)+\frac{1}{2}\left(\frac{\Theta_{12}^{\prime \prime}}{\Theta_{12}}-\frac{\Theta^{\prime \prime}}{\Theta}\right)\left(\mathrm{d} \mathbf{v}\left(z^{\prime}\right)\right)^{2} \\
& \frac{E_{12}}{E_{1 z^{\prime}} E_{z^{\prime} 2}} \frac{\Theta_{1 z^{\prime}} \Theta_{z^{\prime} 2}}{\Theta_{12}}\left\{\frac{1}{2}\left(\frac{\Theta_{1 z^{\prime}}^{\prime}}{\Theta_{1 z}}+\frac{\Theta_{z^{\prime 2}}^{\prime}}{\Theta_{z^{\prime} 2}}\right) \mathrm{d} \mathbf{v}\left(z^{\prime}\right)+\frac{1}{2} \mathrm{~d} S_{12}\left(z^{\prime}\right)\right\}+O\left(\xi_{z^{\prime}}^{-1}(z)\right),
\end{aligned}
$$

where $\xi_{z^{\prime}}$ is our notation for a local coordinate centered at $z^{\prime}$. We use again equation (A.1) and find

$$
\begin{aligned}
H_{12}\left(z^{\prime}, z\right)= & \frac{1}{2} \mathrm{~d} S_{12}\left(z^{\prime}\right)^{2}+\left(\frac{\Theta_{12}^{\prime}}{\Theta_{12}}\right) \mathrm{d} \mathbf{v}\left(z^{\prime}\right) \mathrm{d} S_{12}\left(z^{\prime}\right)+\frac{1}{2}\left(\frac{\Theta_{12}^{\prime \prime}}{\Theta_{12}}-\frac{\Theta^{\prime \prime}}{\Theta}\right)\left(\mathrm{d} \mathbf{v}\left(z^{\prime}\right)\right)^{2} \\
& -\left(\mathrm{d} S_{12}\left(z^{\prime}\right)+\left(\frac{\Theta_{12}^{\prime}}{\Theta_{12}}-\frac{\Theta^{\prime}}{\Theta}\right) \mathrm{d} \mathbf{v}(z)\right)\left\{\frac{1}{2}\left(\frac{\Theta_{1 z^{\prime}}^{\prime}}{\Theta_{1 z}}+\frac{\Theta_{z^{\prime} 2}^{\prime}}{\Theta_{z^{\prime} 2}}\right) \mathrm{d} \mathbf{v}\left(z^{\prime}\right)+\frac{1}{2} \mathrm{~d} S_{12}\left(z^{\prime}\right)\right\} \\
& +O\left(\xi_{z^{\prime}}^{-1}(z)\right) \\
= & \left\{-\mathrm{d} S_{12}\left(z^{\prime}\right)\left(-\frac{\Theta_{12}^{\prime}}{\Theta_{12}}-\frac{\Theta^{\prime}}{\Theta}+\frac{\Theta_{1 z^{\prime}}^{\prime}}{\Theta_{1 z^{\prime}}}+\frac{\Theta_{z^{\prime} 2}^{\prime}}{\Theta_{z^{\prime} 2}}\right)\right. \\
& \left.-\left(\frac{\Theta_{12}^{\prime}}{\Theta_{12}}-\frac{\Theta^{\prime}}{\Theta}\right)\left(\frac{\Theta_{1 z^{\prime}}^{\prime}}{\Theta_{1 z^{\prime}}}+\frac{\Theta_{z^{\prime} 2}^{\prime}}{\Theta_{z^{\prime} 2}}\right) \mathrm{d} \mathbf{v}\left(z^{\prime}\right)+\left(\frac{\Theta_{12}^{\prime \prime}}{\Theta_{12}}-\frac{\Theta^{\prime \prime}}{\Theta}\right) \mathrm{d} \mathbf{v}\left(z^{\prime}\right)\right\} \frac{\mathrm{d} \mathbf{v}\left(z^{\prime}\right)}{2} \\
& +O\left(\xi_{z^{\prime}}^{-1}(z)\right) .
\end{aligned}
$$

We can rearrange the terms

$$
\begin{aligned}
H_{12}\left(z^{\prime}, z\right)= & \left\{-\left[\mathrm{d} S_{12}\left(z^{\prime}\right)+\left(\frac{\Theta_{12}^{\prime}}{\Theta_{12}}-\frac{\Theta^{\prime}}{\Theta}\right) \mathrm{d} \mathbf{v}\left(z^{\prime}\right)\right]\left(\frac{\Theta_{1 z^{\prime}}^{\prime}}{\Theta_{1 z^{\prime}}}+\frac{\Theta_{z^{\prime} 2}^{\prime}}{\Theta_{z^{\prime} 2}}-\frac{\Theta_{12}^{\prime}}{\Theta_{12}}-\frac{\Theta^{\prime}}{\Theta}\right)\right. \\
& \left.+\left(\frac{\Theta_{12}^{\prime}}{\Theta_{12}}-\frac{\Theta^{\prime}}{\Theta}\right)^{\prime} \mathrm{d} \mathbf{v}\left(z^{\prime}\right)\right\} \frac{\mathrm{d} \mathbf{v}\left(z^{\prime}\right)}{2}+O\left(\xi_{z^{\prime}}^{-1}(z)\right),
\end{aligned}
$$

and according to equation (A.3), we see that $H_{12}\left(z^{\prime}, z\right) \in O\left(\xi_{z^{\prime}}^{-1}(z)\right)$. Therefore, $H_{12}\left(z^{\prime}, z\right)$ is a meromorphic function whose only singularity is a pole at most simple at $z=z^{\prime}$. But 
a meromorphic function cannot have a single simple pole, so $H_{12}\left(z, z^{\prime}\right)$ must be holomorphic, and we can write it

$$
H_{12}\left(z, z^{\prime}\right)=h_{12}\left(z^{\prime}\right) \mathrm{d} \mathbf{v}(z)
$$

Since the prefactor $h_{12}\left(z^{\prime}\right)$ is independent of $z$, we may compute it by specializing to $z=z_{1}$ in $H_{12}\left(z, z_{1}\right)$ defined from equation (A.2). Doing so, we obtain

$$
\begin{aligned}
& h_{12}\left(z^{\prime}\right) \mathrm{d} \mathbf{v}\left(z_{1}\right)=\frac{1}{2}\left(\mathrm{~d} S_{12}\left(z^{\prime}\right)\right)^{2} B\left(z^{\prime}, z_{1}\right)+\left(\frac{\Theta_{12}^{\prime}}{\Theta_{12}}\right) \mathrm{d} \mathbf{v}\left(z^{\prime}\right) \mathrm{d} S_{12}\left(z^{\prime}\right) B\left(z^{\prime}, z_{1}\right) \\
& +\frac{1}{2}\left(\frac{\Theta_{12}^{\prime \prime}}{\Theta_{12}}-\frac{\Theta^{\prime \prime}}{\Theta}\right)\left(\mathrm{d} \mathbf{v}\left(z^{\prime}\right)\right)^{2} B\left(z^{\prime}, z_{1}\right)+\frac{1}{2}\left(\frac{\Theta_{12}^{\prime \prime}}{\Theta_{12}}\right)^{\prime}\left(\mathrm{d} \mathbf{v}\left(z^{\prime}\right)\right)^{3} \mathrm{~d} \mathbf{v}\left(z_{1}\right) \\
& +\frac{1}{2}\left(\frac{\Theta_{12}^{\prime}}{\Theta_{12}}\right)^{\prime} \mathrm{d} \mathbf{v}\left(z^{\prime}\right)\left(\mathrm{d} S_{12}\left(z^{\prime}\right)\right)^{2} \mathrm{~d} \mathbf{v}\left(z_{1}\right)+\frac{1}{6}\left(\frac{\Theta_{12}^{\prime \prime \prime}}{\Theta_{12}}-\frac{\Theta^{\prime \prime \prime}}{\Theta}\right)^{\prime}\left(\mathrm{d} \mathbf{v}\left(z^{\prime}\right)\right)^{3} \mathrm{~d} \mathbf{v}\left(z_{1}\right) \\
& -\mathrm{d}_{z=z_{1}}\left[\frac{1}{2}\left(\frac{\Theta_{1 z}^{\prime}}{\Theta_{1 z}}\left(\mathrm{~d} S_{1 z}\left(z^{\prime}\right)\right)^{2}+\frac{\Theta_{z 2}^{\prime}}{\Theta_{z 2}}\left(\mathrm{~d} S_{z 2}\left(z^{\prime}\right)\right)^{2}-\frac{\Theta_{12}^{\prime}}{\Theta_{12}}\left(\mathrm{~d} S_{12}\left(z^{\prime}\right)\right)^{2}\right) \mathrm{d} \mathbf{v}\left(z^{\prime}\right)\right. \\
& +\frac{1}{2}\left(\frac{\Theta_{1 z}^{\prime \prime}}{\Theta_{1 z}} \mathrm{~d} S_{1 z}\left(z^{\prime}\right)+\frac{\Theta_{z 2}^{\prime \prime}}{\Theta_{z 2}} \mathrm{~d} S_{z 2}\left(z^{\prime}\right)-\frac{\Theta_{12}^{\prime \prime}}{\Theta_{12}} \mathrm{~d} S_{12}\left(z^{\prime}\right)\right)\left(\mathrm{d} \mathbf{v}\left(z^{\prime}\right)\right)^{2} \\
& \left.+\frac{1}{6}\left(\frac{\Theta_{1 z}^{\prime \prime \prime}}{\Theta_{1 z}}+\frac{\Theta_{z 2}^{\prime \prime \prime}}{\Theta_{z 2}}-\frac{\Theta_{12}^{\prime \prime \prime}}{\Theta_{12}}-\frac{\Theta^{\prime \prime \prime}}{\Theta}\right)\left(\mathrm{d} \mathbf{v}\left(z^{\prime}\right)\right)^{3}-\frac{1}{2} \mathrm{~d} S_{1 z}\left(z^{\prime}\right) \mathrm{d} S_{z 2}\left(z^{\prime}\right) \mathrm{d} S_{12}\left(z^{\prime}\right)\right] \\
& =\frac{1}{2}\left(\mathrm{~d} S_{12}\left(z^{\prime}\right)\right)^{2} B\left(z^{\prime}, z_{1}\right)+\left(\frac{\Theta_{12}^{\prime}}{\Theta_{12}}\right) \mathrm{d} \mathbf{v}\left(z^{\prime}\right) \mathrm{d} S_{12}\left(z^{\prime}\right) B\left(z^{\prime}, z_{1}\right) \\
& +\frac{1}{2}\left(\frac{\Theta_{12}^{\prime \prime}}{\Theta_{12}}-\frac{\Theta^{\prime \prime}}{\Theta}\right)\left(\mathrm{d} \mathbf{v}\left(z^{\prime}\right)\right)^{2} B\left(z^{\prime}, z_{1}\right)+\frac{1}{2}\left(\frac{\Theta_{12}^{\prime \prime}}{\Theta_{12}}\right)^{\prime}\left(\mathrm{d} \mathbf{v}\left(z^{\prime}\right)\right)^{3} \mathrm{~d} \mathbf{v}\left(z_{1}\right) \\
& +\frac{1}{2}\left(\frac{\Theta_{12}^{\prime}}{\Theta_{12}}\right)^{\prime} \mathrm{d} \mathbf{v}\left(z^{\prime}\right)\left(\mathrm{d} S_{12}\left(z^{\prime}\right)\right)^{2} \mathrm{~d} \mathbf{v}\left(z_{1}\right)+\frac{1}{6}\left(\frac{\Theta_{12}^{\prime \prime \prime}}{\Theta_{12}}-\frac{\Theta^{\prime \prime \prime}}{\Theta}\right)^{\prime}\left(\mathrm{d} \mathbf{v}\left(z^{\prime}\right)\right)^{3} \mathrm{~d} \mathbf{v}\left(z_{1}\right) \\
& -\left[\left(\frac{\Theta_{12}^{\prime}}{\Theta_{12}}\right) B\left(z_{1}, z^{\prime}\right) \mathrm{d} \mathbf{v}\left(z^{\prime}\right) \mathrm{d} S_{12}\left(z^{\prime}\right)+\frac{1}{2}\left(\frac{\Theta_{12}^{\prime}}{\Theta_{12}}\right)^{\prime} \mathrm{d} \mathbf{v}\left(z^{\prime}\right) \mathrm{d} \mathbf{v}\left(z_{1}\right)\left(\mathrm{d} S_{12}\left(z^{\prime}\right)\right)^{2}\right. \\
& +\frac{1}{2}\left(\frac{\Theta_{12}^{\prime \prime}}{\Theta_{12}}-\frac{\Theta^{\prime \prime}}{\Theta}\right)\left(\mathrm{d} \mathbf{v}\left(z^{\prime}\right)\right)^{2} B\left(z_{1}, z^{\prime}\right)+\frac{1}{2}\left(\frac{\Theta_{12}^{\prime \prime}}{\Theta_{12}}\right)^{\prime}\left(\mathrm{d} \mathbf{v}\left(z^{\prime}\right)\right)^{2} \mathrm{~d} \mathbf{v}\left(z_{1}\right) \mathrm{d} S_{12}\left(z^{\prime}\right) \\
& \left.+\frac{1}{6}\left(\frac{\Theta_{12}^{\prime \prime \prime}}{\Theta_{12}}-\frac{\Theta^{\prime \prime \prime}}{\Theta}\right)^{\prime}\left(\mathbf{d} v\left(z^{\prime}\right)\right)^{3} \mathrm{~d} \mathbf{v}\left(z_{1}\right)+\frac{1}{2} B\left(z_{1}, z^{\prime}\right)\left(\mathrm{d} S_{12}\left(z^{\prime}\right)\right)^{2}\right]=0 .
\end{aligned}
$$

All the terms eventually cancel each other. Thus $H_{12}\left(z, z^{\prime}\right)=0$, and coming back to equation (A.2), this proves

$$
\frac{1}{N} \delta_{z} \ln \psi\left(z_{1}, z_{2}\right)+\frac{\psi\left(z_{1}, z\right) \psi\left(z, z_{2}\right)}{\psi\left(z_{1}, z_{2}\right)}=o(1 / N)
$$

\section{Acknowledgments}

We thank O. Babelon, M. Bergère, M. Bertola, B. Dubrovin, D. Korotkin, M. Mulase, J.M. Muñoz Porras, N. Orantin, F. Plaza Martin, E. Previato, A. Raimondo, B. Safnuk for fruitful discussions, T. Grava and S. Romano for enlightening discussions concerning dispersionless hierarchies, their dispersive deformations and the role of Whitham equations, and I. Krichever for careful reading, valuable discussions and for pointing out references. This work is partly supported by the ANR project Grandes Matrices Aléatoires ANR-08-BLAN-0311-01, by the 
European Science Foundation through the Misgam program, by the Québec government with the FQRNT, by the Fonds Européen S16905 (UE7 - CONFRA), by the Swiss NSF (no 20002143434) and the ERC AG CONFRA. B.E. thanks the CERN, and G.B. thanks the SISSA for their hospitality while this work was pursued.

\section{References}

[1] Adler M., van Moerbeke P., Matrix integrals, Toda symmetries, Virasoro constraints, and orthogonal polynomials, Duke Math. J. 80 (1995), 863-911, solv-int/9706010.

[2] Akemann G., Pottier A., Ratios of characteristic polynomials in complex matrix models, J. Phys. A: Math. Gen. 37 (2004), L453-L459, math-ph/0404068.

[3] Albeverio S., Pastur L., Shcherbina M., On the $1 / n$ expansion for some unitary invariant ensembles of random matrices, Comm. Math. Phys. 224 (2001), 271-305.

[4] Babelon O., Bernard D., Talon M., Introduction to classical integrable systems, Cambridge Monographs on Mathematical Physics, Cambridge University Press, Cambridge, 2003.

[5] Bergère M., Biorthogonal polynomials for potentials of two variables and external sources at the denominator, hep-th/0404126.

[6] Bergère M., Eynard B., Universal scaling limits of matrix models and ( $p, q)$ Liouville gravity, arXiv:0909.0854.

[7] Bertola M., Boutroux curves with external field: equilibrium measures without a minimization problem, Anal. Math. Phys. 1 (2011), 167-211, arXiv:0705.3062.

[8] Bertola M., Eynard B., Harnad J., Differential systems for biorthogonal polynomials appearing in 2matrix models and the associated Riemann-Hilbert problem, Comm. Math. Phys. 243 (2003), 193-240, nlin.SI/0208002.

[9] Bertola M., Eynard B., Harnad J., Duality, biorthogonal polynomials and multi-matrix models, Comm. Math. Phys. 229 (2002), 73-120, nlin.SI/0108049.

[10] Bertola M., Eynard B., Harnad J., Partition functions for matrix models and isomonodromic tau functions, J. Phys. A: Math. Gen. 36 (2003), 3067-3083, nlin.SI/0204054.

[11] Bertola M., Eynard B., Harnad J., Semiclassical orthogonal polynomials, matrix models and isomonodromic tau functions, Comm. Math. Phys. 263 (2006), 401-437, nlin.SI/0410043.

[12] Bertola M., Eynard B., Harnad J., The duality of spectral curves that arises in two-matrix models, Theoret. Math. Phys. 134 (2003), 27-38, nlin.SI/0112006.

[13] Bertola M., Gekhtman M., Effective inverse spectral problem for rational Lax matrices and applications, Int. Math. Res. Not. 2007 (2007), no. 23, rnm103, 39 pages, arXiv:0705.0120.

[14] Bertola M., Marchal O., The partition function of the two-matrix model as an isomonodromic $\tau$ function, J. Math. Phys. 50 (2009), 013529, 17 pages, arXiv:0809.3367.

[15] Bertola M., Mo M.Y., Commuting difference operators, spinor bundles and the asymptotics of orthogonal polynomials with respect to varying complex weights, Adv. Math. 220 (2009), 154-218, math-ph/0605043.

[16] Bleher P., Its A., Semiclassical asymptotics of orthogonal polynomials, Riemann-Hilbert problem, and universality in the matrix model, Ann. of Math. (2) 150 (1999), 185-266, math-ph/9907025.

[17] Bonnet G., David F., Eynard B., Breakdown of universality in multi-cut matrix models, J. Phys. A: Math. Gen. 33 (2000), 6739-6768, cond-mat/0003324.

[18] Borot G., Eynard B., All order asymptotics of hyperbolic knot invariants from non-perturbative topological recursion of $A$-polynomials, arXiv:1205.2261.

[19] Borot G., Guionnet A., Asymptotic expansion of $\beta$ matrix models in the one-cut regime, Comm. Math. Phys., to appear, arXiv:1107.1167.

[20] Buchstaber V.M., Krichever I.M., Integrable equations, addition theorems and the Riemann-Schottky problem, Russian Math. Surveys 61 (2006), 19-78.

[21] Chekhov L., Eynard B., Marchal O., Topological expansion of the $\beta$-ensemble model and quantum algebraic geometry in the sectorwise approach, Theoret. Math. Phys. 166 (2011), 141-185, arXiv:1009.6007.

[22] Chekhov L., Eynard B., Marchal O., Topological expansion of the Bethe ansatz, and quantum algebraic geometry, arXiv:0911.1664. 
[23] Chen Y., Ismail M.E.H., Ladder operators and differential equations for orthogonal polynomials, J. Phys. A: Math. Gen. 30 (1997), 7817-7829.

[24] Deift P., Kriecherbauer T., McLaughlin K.D. T-R, Venakides S., Zhou X., Strong asymptotics of orthogonal polynomials with respect to exponential weights, Comm. Pure Appl. Math. 52 (1999), 1491-1552.

[25] Deift P., Venakides S., Zhou X., New results in small dispersion KdV by an extension of the steepest descent method for Riemann-Hilbert problems, Int. Math. Res. Not. 1997 (1997), no. 6, 285-299, arXiv:0705.0120.

[26] Deift P., Zhou X., A steepest descent method for oscillatory Riemann-Hilbert problems. Asymptotics for the MKdV equation, Ann. of Math. (2) 137 (1993), 295-368, math.AP/9201261.

[27] Dijkgraaf R., Vafa C., On geometry and matrix models, Nuclear Phys. B 644 (2002), 21-39, hep-th/0207106.

[28] Douglas M.R., Shenker S.H., Strings in less than one dimension, Nuclear Phys. B 335 (1990), 635-654.

[29] Dubrovin B.A., Geometry of 2D topological field theories, in Integrable Systems and Quantum Groups (Montecatini Terme, 1993), Lecture Notes in Math., Vol. 1620, Springer, Berlin, 1996, 120-348, hep-th/9407018.

[30] Dubrovin B.A., Integrable systems and classification of 2-dimensional topological field theories, in Integrable Systems (Luminy, 1991), Progr. Math., Vol. 115, Birkhäuser Boston, Boston, MA, 1993, 313-359, hepth/9209040.

[31] Dubrovin B.A., Theta functions and non-linear equations, Russian Math. Surveys 36 (1981), no. 2, 11-92.

[32] Dubrovin B.A., Krichever I.M., Novikov S.P., Integrable systems. I, in Dynamical systems, IV, Encyclopaedia Math. Sci., Vol. 4, Springer, Berlin, 2001, 177-332.

[33] Dubrovin B.A., Zhang Y., Normal forms of hierarchies of integrable PDEs, Frobenius manifolds and GromovWitten invariants, math.DG/0108160.

[34] Ercolani N., McLaughlin K.D. T-R, Asymptotics of the partition function for random matrices via RiemannHilbert techniques and applications to graphical enumeration, Int. Math. Res. Not. 2003 (2003), no. 14, 755-820, math-ph/0211022.

[35] Eynard B., Large $N$ expansion of convergent matrix integrals, holomorphic anomalies, and background independence, J. High Energy Phys. 2009 (2009), no. 3, 003, 20 pages, arXiv:0802.1788.

[36] Eynard B., Topological expansion for the 1-Hermitian matrix model correlation functions, J. High Energy Phys. 2004 (2004), no. 11, 031, 35 pages, hep-th/0407261.

[37] Eynard B., Mariño M., A holomorphic and background independent partition function for matrix models and topological strings, J. Geom. Phys. 61 (2011), 1181-1202, arXiv:0810.4273.

[38] Eynard B., Orantin N., Invariants of algebraic curves and topological expansion, Commun. Number Theory Phys. 1 (2007), 347-452, math-ph/0702045.

[39] Eynard B., Orantin N., Topological expansion of mixed correlations in the Hermitian 2-matrix model and $x$-y symmetry of the $F_{g}$ algebraic invariants, J. Phys. A: Math. Theor. 41 (2008), 015203, 28 pages, arXiv:0705.0958.

[40] Farkas H.M., Kra I., Riemann surfaces, Graduate Texts in Mathematics, Vol. 71, 2nd ed., Springer-Verlag, New York, 1992.

[41] Fay J.D., Theta functions on Riemann surfaces, Lecture Notes in Mathematics, Vol. 352, Springer-Verlag, Berlin, 1973.

[42] Flaschka H., Forest M.G., McLaughlin D.W., Multiphase averaging and the inverse spectral solution of the Korteweg-de Vries equation, Comm. Pure Appl. Math. 33 (1980), 739-784.

[43] Fokas A.S., Its A.R., Kapaev A.A., Novokshenov V.Yu., Painlevé transcendents. The Riemann-Hilbert approach, Mathematical Surveys and Monographs, Vol. 128, American Mathematical Society, Providence, RI, 2006.

[44] Fyodorov Y.V., Strahov E., An exact formula for general spectral correlation function of random Hermitian matrices, J. Phys. A: Math. Gen. 36 (2003), 3203-3213, math-ph/0204051.

[45] Gurevich A., Pitaevskii L., Nonstationary structure of a collisionless shock wave, Soviet Phys. JEPT 38 (1974), 291-297.

[46] Harnad J., Tracy C.A., Widom H., Hamiltonian structure of equations appearing in random matrices, in Low-Dimensional Topology and Quantum Field Theory (Cambridge, 1992), NATO Adv. Sci. Inst. Ser. B Phys., Vol. 315, Editor H. Osborn, Plenum, New York, 1993, 231-245, hep-th/9301051.

[47] Hirota R., Exact solution of the Korteweg-de Vries equation for multiple collisions of solitons, Phys. Rev. Lett. 27 (1971), 1192-1194. 
[48] Hitchin N., Stable bundles and integrable systems, Duke Math. J. 54 (1987), 91-114.

[49] Its A.R., Izergin A.G., Korepin V.E., Slavnov N.A., Differential equations for quantum correlation functions, Internat. J. Modern Phys. B 4 (1990), 1003-1037.

[50] Its A.R., Kitaev A.V., Fokas A.S., An isomonodromic approach in the theory of two-dimensional quantum gravity, Russian Math. Surveys 45 (1990), no. 6, 155-157.

[51] Its A.R., Matveev V.B., Hill's operator with finitely many gaps, Funct. Anal. Appl. 9 (1975), 65-66.

[52] Its A.R., Matveev V.B., Schrödinger operators with finite-gap spectrum and $N$-soliton solutions of the Korteweg-de Vries equation, Theoret. Math. Phys. 23 (1975), 343-355.

[53] Jimbo M., Miwa T., Monodromy preserving deformation of linear ordinary differential equations with rational coefficients. II, Phys. D 2 (1981), 407-448.

[54] Jimbo M., Miwa T., Monodromy preserving deformation of linear ordinary differential equations with rational coefficients. III, Phys. D 4 (1981), 26-46.

[55] Jimbo M., Miwa T., Solitons and infinite-dimensional Lie algebras, Publ. Res. Inst. Math. Sci. 19 (1983), 943-1001.

[56] Jimbo M., Miwa T., Ueno K., Monodromy preserving deformation of linear ordinary differential equations with rational coefficients. I. General theory and $\tau$-function, Phys. D 2 (1981), 306-352.

[57] Kharchev S., Marshakov A., Mironov A., Morozov A., Zabrodin A., Unification of all string models with $c<1$, Phys. Lett. B 275 (1992), 311-314, hep-th/9111037.

[58] Kokotov A., Korotkin D., Invariant Wirtinger projective connection and tau-functions on spaces of branched coverings, in Superintegrability in Classical and Quantum Systems, CRM Proc. Lecture Notes, Vol. 37, Amer. Math. Soc., Providence, RI, 2004, 91-97, math-ph/0310008.

[59] Korotkin D., Matrix Riemann-Hilbert problems related to branched coverings of $\mathbb{C P}^{1}$, in Factorization and Integrable Systems (Faro, 2000), Oper. Theory Adv. Appl., Vol. 141, Birkhäuser, Basel, 2003, 103-129, math-ph/0106009.

[60] Korotkin D., Solution of matrix Riemann-Hilbert problems with quasi-permutation monodromy matrices, Math. Ann. 329 (2004), 335-364, math-ph/0306061.

[61] Kostov I., Bilinear functional equations in 2d quantum gravity, hep-th/9602117.

[62] Krichever I.M., Integration of nonlinear equations by the methods of algebraic geometry, Funct. Anal. Appl. 11 (1977), 12-26.

[63] Krichever I.M., Methods of algebraic geometry in the theory of non-linear equations, Russian Math. Surveys 32 (1977), no. 6, 185-213.

[64] Krichever I.M., Perturbation theory in periodic problems for two-dimensional integrable systems, Sov. Sci. Rev. Sect. C 9 (1992), no. 2, 1-103.

[65] Krichever I.M., The $\tau$-function of the universal Whitham hierarchy, matrix models and topological field theories, Comm. Pure Appl. Math. 47 (1994), 437-475, hep-th/9205110.

[66] Lax P., Levermore C., The small dispersion limit of the Korteweg-de Vries equation. I, Comm. Pure Appl. Math. 36 (1983), 253-290.

[67] Lax P., Levermore C., The small dispersion limit of the Korteweg-de Vries equation. II, Comm. Pure Appl. Math. 36 (1983), 571-593.

[68] Lax P., Levermore C., The small dispersion limit of the Korteweg-de Vries equation. III, Comm. Pure Appl. Math. 36 (1983), 809-830.

[69] Mehta M.L., Random matrices, Pure and Applied Mathematics (Amsterdam), Vol. 142, 3rd ed., Elsevier/Academic Press, Amsterdam, 2004.

[70] Moore G., Geometry of the string equations, Comm. Math. Phys. 133 (1990), 261-304.

[71] Mumford D., Tata lectures on theta. I, Progress in Mathematics, Vol. 28, Birkhäuser Boston Inc., Boston, MA, 1983.

[72] Mumford D., Tata lectures on theta. II, Progress in Mathematics, Vol. 43, Birkhäuser Boston Inc., Boston, MA, 1984.

[73] Mumford D., Tata lectures on theta. III, Progress in Mathematics, Vol. 97, Birkhäuser Boston Inc., Boston, MA, 1991.

[74] Rauch H.E., Weierstrass points, branch points, and moduli of Riemann surfaces, Comm. Pure Appl. Math. 12 (1959), 543-560. 
[75] Sato M., Sato Y., Soliton equations as dynamical systems on infinite-dimensional Grassmann manifold, in Nonlinear Partial Differential Equations in Applied Science (Tokyo, 1982), North-Holland Math. Stud., Vol. 81, North-Holland, Amsterdam, 1983, 259-271.

[76] Segal G.B., Loop groups, in Workshop Bonn 1984 (Bonn, 1984), Lecture Notes in Math., Vol. 1111, Springer, Berlin, 1985, 155-168.

[77] Shabat A.B., Zakharov V.E., A scheme for integrating the nonlinear equations of mathematical physics by the method of the inverse scattering problem. I, Funct. Anal. Appl. 11 (1977), 226-235.

[78] van Moerbeke P., The spectrum of random matrices and integrable systems, in in Group21, Physical Applications and Mathematical Aspects of Geometry, Groups and Algebras, Vol. II, World Scientific, Singapore, 1997, 835-852, solv-int/9706009.

[79] Venakides S., The generation of modulated wavetrains in the solution of the Korteweg-de Vries equation, Comm. Pure Appl. Math. 38 (1985), 883-909.

[80] Venakides S., The Korteweg-de Vries equation with small dispersion: higher order Lax-Levermore theory, Comm. Pure Appl. Math. 43 (1990), 335-361.

[81] Whitham G.B., Linear and nonlinear waves, Pure and Applied Mathematics, Wiley Interscience, New York, 1974. 